\title{
A!
}

This is an electronic reprint of the original article.

This reprint may differ from the original in pagination and typographic detail.

Halme, Janne; Vahermaa, Paula; Miettunen, Kati; Lund, Peter

\section{Device physics of dye solar cells}

Published in:

Advanced Materials

DOI:

10.1002/adma.201000726

Published: 15/09/2010

Document Version

Peer reviewed version

Please cite the original version:

Halme, J., Vahermaa, P., Miettunen, K., \& Lund, P. (2010). Device physics of dye solar cells. Advanced Materials, 22(35), E210-E234. https://doi.org/10.1002/adma.201000726

This material is protected by copyright and other intellectual property rights, and duplication or sale of all or part of any of the repository collections is not permitted, except that material may be duplicated by you for your research use or educational purposes in electronic or print form. You must obtain permission for any other use. Electronic or print copies may not be offered, whether for sale or otherwise to anyone who is not an authorised user. 
DOI: 10.1002/adma.201000726

Device physics of dye solar cells

By Janne Halme*, Paula Vahermaa, Kati Miettunen, Peter Lund

[*] Dr. Janne Halme

Aalto University

Department of Applied Physics

P.O.Box 15100

FI-00076 AALTO, Finland

E-mail: janne.halme@tkk.fi

Keywords: equivalent circuit, dye-sensitized, electrochemistry, photovoltaic,

differential resistance

\begin{abstract}
Design of new materials for nanostructured dye solar cells (DSC) requires understanding the link between the material properties and cell efficiency. This paper gives an overview of the fundamental and practical aspects of the modeling and characterization of DSCs, and integrates the knowledge into a user-friendly DSC device model. Starting from basic physical and electrochemical concepts, mathematical expressions for the IV curve and differential resistance of all resistive cell components are derived and their relation to electrochemical impedance spectroscopy (EIS) is explained. The current understanding of the associated physics is discussed in detail and clarified. It is shown how the model parameters can be determined from complete DSCs by current dependent EIS and incident-photon-to-collectedelectron (IPCE) measurements, supplemented by optical characterization, and used to quantify performance losses in DSCs. The paper aims to give a necessary theoretical background and practical guidelines for establishing an effective feedback-loop for DSC testing and development.
\end{abstract}




\section{Introduction}

The dye solar cell (DSC) $)^{[1-3]}$ is an electrochemical device that uses light-absorbing dye molecules adsorbed on semiconductor nanoparticles to generate electricity from the sunlight. They are promising for low-cost solar electricity generation due to their cheap printable materials and simple manufacturing techniques. Small cells reach over $10 \%$ efficiency, ${ }^{[4-8]}$ endure more than 1000 hours of accelerated aging at $80{ }^{\circ} \mathrm{C}$ in the dark and continuous illumination of $1000 \mathrm{~W} \mathrm{~m}^{-2}$ visible light at $60^{\circ} \mathrm{C}$ with negligible or only minor performance degradation. ${ }^{[7,8]}$ Remarkable stability over $20,000 \mathrm{~h}$ at $55^{\circ} \mathrm{C}$ under 0.8 Sun illumination has also been reported. ${ }^{[9]}$

The present focus in the development of DSCs is on finding cell materials and manufacturing techniques that give higher conversion efficiency, lower costs, and longer operating lifetime. In this process, quantitative understanding of the influence of the cell materials and components on the cell efficiency is essential, as it provides a basis for identifying which factors are the most significant limitations to the efficiency of a DSC sample under investigation. This quantitative understanding can be demonstrated only by expressing it in the form of a mathematical model that describes the steady state current - voltage (IV) curve of the solar cell at different operating conditions of practical interest, using a fixed set of physical parameters.

In the simplest case, the IV curve of DSC can be expressed using an equivalent circuit model adopted from silicon solar cells. While this model fits the IV curve of a reasonably high efficiency DSC well, ${ }^{[10-14]}$ it does not describe how the model parameters are related to physical and electrochemical processes taking place in an operating DSC, and hence, it cannot be used to predict the influence of improvements made in the properties of the cell materials. This can only be done by physical modeling. 
Physical models that predict the IV curve can be categorized as numerical ${ }^{[15-19]}$ or analytical $^{[20-23]}$ models. The numerical models have the advantage that the physical principles of DSC operation can be correctly described, usually as a system of partial differential equations. ${ }^{[15]}$ The numerical models can be powerful simulation tools, but with large number of independent parameters, they tend to be too complex and computationally heavy to lend themselves to routine analysis by fitting the model to experimental data. Complexity is an issue in some analytical models as well. ${ }^{[20,22,23]} \mathrm{A}$ compromise in this respect is the simple numerical model presented recently by Villanueva et al.. ${ }^{[19]}$ The parameters of this model are estimated by adjusting them recursively to a set of steady state and dynamic measurement data. ${ }^{[19]}$ However, to finally fit their model to a measured solar cell IV curve, the authors use a constant series resistance as an empirical fitting parameter in an iterative fitting process that involves repetitive numerical solution of the model.

In this paper, we share the spirit of Villanueva et al., ${ }^{[19]}$ who want to "fill the gap between theoreticians and experimentalists", and construct a physical DSC device model that can be verified by widely available experimental techniques. In our case, we build the model around steady state parameters that can be determined by electrochemical impedance spectroscopy (EIS), optical characterization, and spectral incident-photon-to-collected-electron (IPCE) measurements in addition to photovoltaic IV characterization. More specifically, our objective was to construct a mathematical model of DSC that

1. Using physical parameters, predicts quantitatively the IV curve of DSC and the factors that limit its energy conversion efficiency.

2. Instead of numerical simulations, is based on analytical expressions that facilitate spreadsheet calculations. 
3. Is based on with the widely used equivalent circuit impedance model of DSC, ${ }^{[24-27]}$ and hence, can be verified by and fitted to experimental EIS data.

The advantage of EIS over other dynamic DSC characterization techniques is that it gives readily information on the internal cell resistances that are directly related to the steady state IV curve of the cell. ${ }^{[28,29]}$ Hence, with EIS there is no need to "fit" a series resistance parameter in order to make the model match with the measured IV data, but instead the internal cell resistances contributing to it can be measured. We build the connection between the cell impedance and IV curve similar to Fabregat-Santiago et al., ${ }^{[26]}$ with the difference that we emphasize the importance and advantage of analyzing the impedance data and carrying out the resistance modeling as a function of cell current density ${ }^{[29,30]}$ instead of cell voltage. ${ }^{[26,}$ 31, 32] We go also further in the physical modeling by deriving the current and voltage dependence of all the resistive equivalent circuit components, and their IV curves, using basic semiconductor device physics and electrochemistry. For the photoelectrode, we use the standard electron diffusion model, ${ }^{[33]}$ and discuss its relation to transmission line impedance model as well as the interpretation of the ideality factor and the effects of electron trapping. For the counter electrode, we start from the current - overpotential equation, couple it to a simple model of ionic diffusion in the electrolyte, separate contributions from the charge transfer and diffusion resistance, and discuss their relation to EIS as well as the effects of transport mechanism and electrode porosity.

As a whole, the paper provides a comprehensive description and review of the physical and electrochemical operation of DSC integrated in the form of a complete DSC device model that can be coupled to data from readily available experimental techniques and used to analyze and predict the photovoltaic performance of DSCs. The model is anticipated to serve as a useful reference and a practical analysis tool for a materials scientists and engineers working on experimental DSC material and device development. Theoreticians who aim to 
develop more detailed physical models will find the present model useful for validation and comparison purposes and for determining initial values for their model parameters based on experimental data.

We start the paper with a description of DSC operation and its simple diode modeling, and present thereafter a compact review of the impedance modeling and characterization of DSCs pointing out the relation between impedance and IV curve. The physical modeling begins with photocurrent generation which is followed by IV and resistance modeling of the photoelectrode, the counter electrode, and the Ohmic series resistances, each discussed separately and demonstrated with experimental data and model calculations. Finally, the model is summarized and applied to analyze fill factor losses in a typical DSC.

\section{Theoretical background}

\subsection{Structure and operation of the dye solar cell}

The structure of a typical DSC is shown Figure 1. The photoelectrode (photoanode) is a 10 $\mu \mathrm{m}$ thick nanoporous film of interconnected $\mathrm{TiO}_{2}$ nanoparticles with ca. $20 \mathrm{~nm}$ diameter that are covered with a monolayer of light absorbing dye molecules. The film is deposited on a transparent conductive oxide (TCO) coated glass or plastic substrate. The nanoporous structure of the $\mathrm{TiO}_{2}$ film provides high internal surface area to accommodate sufficient amount of dye for efficient light absorption. It also ensures that each dye molecule is in direct contact with both the $\mathrm{TiO}_{2}$ and the electrolyte that fills the pores of the film. The counter electrode (cathode) is a similar TCO glass substrate but is coated with platinum catalyst particles. The electrodes are attached together with a thermoplastic polymer film that functions both as an edge sealant and spacer forming a sandwich-like thin layer electrochemical cell where an electrolyte layer fills the gap between the electrodes. 
The operating principle of DSC is summarized in Figure 2. When the dye absorbs a photon an electron is excited from a low-energy state (highest occupied molecular orbital, HOMO) to a high-energy state (lowest unoccupied molecular orbital, LUMO) of the molecule (1). This is followed by electron injection to the $\mathrm{TiO}_{2}$ conduction band (2), and the oxidized dye is regenerated by electron capture from the redox electrolyte (3). The injected electron travels by diffusion in the $\mathrm{TiO}_{2}$ film until it finds it way to the substrate contact where it is released to the external electrical circuit (4). The electron is returned to the cell via an electrolyte reduction reaction at the counter electrode (5). The electrical circuit of the cell is completed by ionic transport of the redox pair in the electrolyte (6).

This operating cycle can be summarized as follows.

$\underline{\text { Photoelectrode }}$

$$
\begin{array}{ll}
\mathrm{S}+h v \rightarrow \mathrm{S}^{*} & \text { light absorption by the dye } \\
\mathrm{S}^{*} \rightarrow \mathrm{S}^{+}+\mathrm{e}^{-}\left(\mathrm{TiO}_{2}\right) & \text { electron injection } \\
\mathrm{e}^{-}\left(\mathrm{TiO}_{2}\right) \rightarrow \mathrm{e}^{-}(\mathrm{PE}) & \text { electron transport } \\
2 \mathrm{~S}^{+}+3 \mathrm{I}^{-}(\mathrm{PE}) \rightarrow 2 \mathrm{~S}+\mathrm{I}_{3}^{-}(\mathrm{PE}) & \text { dye regeneration }
\end{array}
$$

\section{$\underline{\text { Counter electrode }}$}

$\mathrm{I}_{3}^{-}(\mathrm{CE})+2 \mathrm{e}^{-}(\mathrm{CE}) \rightarrow 3 \mathrm{I}^{-}(\mathrm{CE}) \quad$ overall charge transfer reaction

\section{Electrolyte}

$$
\begin{array}{ll}
3 \mathrm{I}^{-}(\mathrm{CE}) \rightarrow 3 \mathrm{I}^{-}(\mathrm{PE}) & \text { Iodide and tri-iodide diffusion } \\
\mathrm{I}_{3}^{-}(\mathrm{PE}) \rightarrow \mathrm{I}_{3}^{-}(\mathrm{CE}) &
\end{array}
$$

\section{Total reactions:}

$$
\begin{array}{ll}
3 \mathrm{I}^{-}(\mathrm{PE})+2 h v \rightarrow \mathrm{I}_{3}^{-}(\mathrm{PE})+2 \mathrm{e}^{-}\left(\mathrm{TiO}_{2}\right) & \text { Photoelectrode } \\
\mathrm{e}^{-}(\mathrm{CE})+h v \rightarrow \mathrm{e}^{-}(\mathrm{PE}) & \text { Cell }
\end{array}
$$

The objective of the physical modeling of the DSC operation is to explain how these processes are related to the IV curve of the cell. 


\subsection{Basic diode model of a solar cell}

The shape of the IV curve of a high efficient DSC can be usually reproduced quite well ${ }^{[10-14]}$ with the simple diode equivalence circuit model of Figure 3 corresponding to the following IV equation

$$
i_{\mathrm{CELL}}=i_{\mathrm{ph}}-i_{0}\left(e^{\frac{-q_{\mathrm{e}}\left(V_{\mathrm{CEL}}+i_{\mathrm{CEL}} R_{\mathrm{S}}\right)}{m k_{\mathrm{B}} T}}-1\right)+\frac{V_{\mathrm{CELL}}+i_{\mathrm{CELL}} R_{\mathrm{S}}}{R_{\mathrm{sh}}}
$$

where $i_{\mathrm{ph}}$ is the photocurrent density modeled as a current source, $i_{0}$ the reverse saturation current density (dark current) of the diode, $k_{\mathrm{B}}$ is the Boltzmann constant, $q_{\mathrm{e}}$ the electron charge, $T$ the absolute cell temperature, $m$ is the diode ideality factor, and $R_{\mathrm{S}}$ and $R_{\mathrm{sh}}$ are the series and shunt resistances of the cell.

The diode model parameters can be determined by fitting the model to a measured solar cell IV curve (Figure 4). However, this gives very limited information on the physical properties that limit the photovoltaic performance of DSC. Moreover, in the fitting process $R_{\mathrm{S}}$ needs to be assumed constant, which is not the case in DSCs, where it includes a contributions from the current dependent resistances of charge transfer and mass transport at the counter electrode, as pointed out by others ${ }^{[10]}$ and discussed in the present paper (Section 5) An access to more detailed electrical description of the DSC operation is provided by electrochemical impedance spectroscopy measurements and impedance modeling.

\subsection{Impedance spectroscopy and equivalent circuit modeling of dye solar cells}

In an EIS measurement the solar cell is set to steady state operating conditions defined by the light intensity and cell temperature and the operating point $\left(V_{\mathrm{CELL}}, i_{\mathrm{CELL}}\right)$ along its IV curve. A small amplitude harmonic AC voltage modulation $V_{\mathrm{AC}}(\omega, t)$ is superimposed on the DC voltage of the cell and the resulting $\mathrm{AC}$ current $i_{\mathrm{AC}}(\omega, t)$ response is measured while scanning 
the modulation frequency $f=\omega / 2 \pi$ over a certain range. This gives the impedance spectrum $Z_{\mathrm{CELL}}(\omega)$ of the cell

$$
Z_{\mathrm{CELL}}(\omega)=\frac{V_{\mathrm{AC}}(\omega, t)}{i_{\mathrm{AC}}(\omega, t)}
$$

Due to the small amplitude of the AC voltage modulation, the impedance can be regarded as the frequency dependent differential resistance of the IV curve. In other words, in the limit of zero frequency, EIS gives the slope of the IV curve at the operating point (Figure 5).

$$
Z_{\mathrm{CELL}}(\omega) \underset{\omega \rightarrow 0}{\longrightarrow} \frac{\partial V_{\mathrm{CELL}}}{\partial i_{\mathrm{CELL}}}=R_{\mathrm{CELL}}
$$

The impedance of DSC can be described effectively and informatively by an equivalent circuit model. ${ }^{[24,26-28,34,35]}$ The equivalent circuit is constructed by assigning different cell components and their interfaces resistances that represent hindrance to charge transport in the materials and charge transfer across their interfaces, while capacitances represent accumulation of charge at these interfaces.

Figure 6a shows the widely used equivalent circuit impedance model of DSC. ${ }^{[25,26,28]}$ The circuit components and their units are:

- $\quad r_{\mathrm{T}}(\Omega \mathrm{m})$ : Resistivity of electron transport in the photoelectrode film, consisting typically of interconnected $\mathrm{TiO}_{2}$ nanoparticles. The total transport resistance of the film is $R_{\mathrm{T}}=r_{\mathrm{T}} d$ $\left(\Omega \mathrm{m}^{2}\right)$, where $d$ is the film thickness.

- $\quad r_{\mathrm{REC}}\left(\Omega \mathrm{m}^{3}\right)$ : Charge transfer (recombination) resistance at the $\mathrm{TiO}_{2} /$ dye/electrolyte interface per unit volume of the electrode. The total recombination resistance of the film is $R_{\mathrm{REC}}=r_{\mathrm{REC}} d^{-1}\left(\Omega \mathrm{m}^{2}\right)$.

- $\quad c_{\mu}\left(\mathrm{F} \mathrm{m}^{-3}\right)$ : Photoelectrode (chemical) capacitance per unit volume. The total capacitance of the film is $C_{\mu}=c_{\mu} d$

- $\quad R_{\mathrm{CO}}\left(\Omega \mathrm{m}^{2}\right)$ and $C_{\mathrm{CO}}\left(\mathrm{F} \mathrm{m}^{-2}\right)$ : Contact resistance and capacitance at the interface between the conducting substrate and the $\mathrm{TiO}_{2}$ photoelectrode film. 
- $\quad R_{\mathrm{SU}}\left(\Omega \mathrm{m}^{2}\right)$ and $C_{\mathrm{SU}}\left(\mathrm{F} \mathrm{m}^{-2}\right)$ : Charge transfer resistance and double layer capacitance at the substrate-electrolyte interface.

- $\quad R_{\mathrm{CE}}\left(\Omega \mathrm{m}^{2}\right)$ and $C_{\mathrm{CE}}\left(\mathrm{F} \mathrm{m}^{-2}\right)$ : Charge transfer resistance and double layer capacitance at the counter electrode-electrolyte interface.

- $Z_{\mathrm{D}}\left(\Omega \mathrm{m}^{2}\right)$ : Mass transport impedance at the counter electrode.

- $\quad R_{\mathrm{S}}\left(\Omega \mathrm{m}^{2}\right)$ : Ohmic series resistance of the cell. The total $R_{\mathrm{S}}$ is the sum of contributions from the sheet resistance of the substrates, resistivity of the electrolyte and electrical contacts and wiring of the cell.

Photocurrent generation $\left(i_{\mathrm{GEN}}, \mathrm{A} \mathrm{m}^{-2}\right)$ is expressed in the equivalent circuit by inserting current source elements $i_{\mathrm{ph}}\left(\mathrm{A} \mathrm{m}^{-3}\right)$ that are distributed along the photoelectrode film (Figure 6a-b). ${ }^{[35,36]}$ With the units indicated above, the parameters $r_{\mathrm{T}}, r_{\mathrm{CT}}$, and $c_{\mu}$, can be regarded as material properties independent of the photoelectrode film thickness. Likewise, using the unit $\Omega \mathrm{cm}^{2}$ for the other resistance parameters makes them independent of the active area of the solar cell. In addition to the simple resistors and capacitors, there are two special impedance components in the model: the so-called transmission line impedance $Z_{\mathrm{TiO} 2}$ of the $\mathrm{TiO}_{2}$ photoelectrode film consisting of the elements $r_{\mathrm{T}}, r_{\mathrm{REC}}$, and $c_{\mu}$, and the mass transport impedance $Z_{\mathrm{D}}$ at the counter electrode. These impedance elements and their frequency dependence are described in Appendix I. Also, the ideal capacitors in the model are often replaced with constant phase elements (Equation A4 in the Appendix) to improve accuracy of the model fit to data. Note that the model neglects electrolyte mass transport and resistivity in the pores of the photoelectrode film. This assumption is discussed in detail in Section 5.5. At the limit of steady state $(\omega=0)$ the total resistance of the cell (Equation 11), corresponding to the equivalent circuit of Figure $6 \mathrm{~d}$ is

$$
R_{\mathrm{CELL}}=R_{\mathrm{S}}+R_{\mathrm{PE}}+R_{\mathrm{D}}+R_{\mathrm{CE}}
$$

where 


$$
R_{\mathrm{PE}}=\frac{R_{\mathrm{SU}}\left(R_{\mathrm{CO}}+R_{\mathrm{TiO} 2}\right)}{R_{\mathrm{SU}}+R_{\mathrm{CO}}+R_{\mathrm{TiO} 2}}
$$

is the overall resistance of the photoelectrode including its substrate and $R_{\mathrm{TiO} 2}$ is the total resistance of the photoelectrode film given by Equation A7 in Appendix I.

The power of EIS for the DSC analysis is illustrated by Figure 5. In a typical DSC, consisting of a nanoporous photoelectrode, liquid electrolyte and a planar counter electrode, the different impedance components of the cell exhibit largely different time constants (characteristic frequencies) and therefore can be seen as separate impedance arcs in the complex plane plot of the cell impedance, at least when the operating point is close to the open circuit conditions. This is enables fractioning the total cell resistance (Equation 12) to partial contributions from the different cell components and interfaces by the EIS measurements.

Since the IV curve is nonlinear, the cell resistance measured by EIS is not constant but a function of the operating point $\left(V_{\mathrm{CELL}}, i_{\mathrm{CELL}}\right)$. In fact, the IV curve can be expressed in terms of the differential cell resistance $R_{\mathrm{CELL}}$, which is the sum of the differential resistances $R_{\mathrm{k}}$ of the individual cell components (Equation 12)

$$
V_{\mathrm{CELL}}\left(i_{\mathrm{CELL}}\right)=V_{\mathrm{OC}}+\int_{0}^{i_{\mathrm{CELL}}} R_{\mathrm{CELL}}(i) d i=V_{\mathrm{OC}}+\int_{0}^{i_{\mathrm{CEL}}} \sum_{k=\mathrm{S}, \mathrm{PE}, \mathrm{D}, \mathrm{CE}} R_{\mathrm{k}}(i) d i
$$

where $i$ is the current density. Note that in our model we use the sign convention that $V_{\mathrm{CELL}}<$ 0 and $i_{\text {CELL }}>0$ at the maximum power point of the cell (See Figure $6 \mathrm{~b}$ ). This means that the open circuit voltage $V_{\mathrm{OC}}<0$ and the short circuit current density $i_{\mathrm{SC}}>0$ in Equation 14, and that all the differential resistances in the model (Equations 12 and 13) are positive. For the current densities the following thus applies in accordance with Figure 6b

$$
i_{\mathrm{CELL}}=i_{\mathrm{TiO} 2}-i_{\mathrm{SU}}=i_{\mathrm{GEN}}-i_{\mathrm{REC}}-i_{\mathrm{SU}}
$$

The resistance - IV relation of Equation 14 is similar to that introduced by Fabregat-Santiago et al. ${ }^{[28]}$ with the difference that here, we choose $V_{\mathrm{OC}}$ instead of $i_{\mathrm{SC}}$ as a point of reference, i.e. 
the known constant for the integration. This is essential for our modeling where we want to include the possibility that the $i_{\mathrm{SC}}$ of the cell is limited by the cell resistances instead of photocurrent generation. In such a case, it is easiest to base the calculations to $V_{\mathrm{OC}}$, since it is defined exclusively by the photoelectrode (see Equation 17 below). Moreover, using $V_{\mathrm{OC}}$ as a reference is a standard way to describe the IV characteristics of an electrochemical cell: $V_{\mathrm{OC}}$ is the light intensity dependent rest potential of the cell. Connecting the cell to an electric load causes electric current to flow in the cell which gives rise to voltage losses $V_{\mathrm{k}}$ that are also called overpotentials or overvoltages, due to the internal cell resistances $R_{\mathrm{k}}$. This decreases the cell voltage from its open circuit value and determines the shape of the solar cell IV curve, and hence its fill factor.

Note that there is also good reason for expressing the resistances as a function of current instead of voltage: In a two-terminal DSC, with no reference electrodes built-in, the voltage over each series connected resistance component (Equation 12) cannot be directly measured, whereas the current through them can, since it equals the external cell current. This means that the internal voltage losses in the cell and their effect on its IV curve can be evaluated based on EIS data measured as a function of the cell current density. ${ }^{[29,30]}$ In our previous paper ${ }^{[29]}$ we carried out the integration in Equation 14 with the help of an empirical analytical expression fitted to the measured $R_{\mathrm{k}}$ vs. $i_{\text {CELL }}$ data, arriving thus to the corresponding $V_{\mathrm{k}}$ vs. $i_{\mathrm{CELL}}$ data, i.e. the IV curve of that component. This analysis method represents a mechanical factoring of the solar cell IV curve to the individual IV curves corresponding to the resistances visible in EIS spectra. This has the advantage that no physical model is needed to carry out the factoring, but at the same time less physical insight is obtained from the analysis.

In this paper, we take an alternative approach: instead of mechanical factoring the IV curve with EIS, we want to understand the physical and electrochemical origin of the internal cell resistance of DSC, and do this by building a mathematical model that predicts the current 
dependence of the resistances $R_{\mathrm{k}}$ in Equation 14. This provides us with a method not only to factor the IV curve, but also to estimate the underlying physical parameters that determine the IV curve of DSC.

Our starting point for the modeling presented in the following Sections is the following equation that expresses the solar cell IV curve as the sum of the partial IV curves that correspond to the differential resistance components in the equivalent circuit model of Figure $6 a$.

$$
V_{\mathrm{CELL}}\left(i_{\mathrm{CELL}}\right)=V_{\mathrm{PE}}\left(i_{\mathrm{CELL}}\right)+\sum_{k \neq \mathrm{PE}} V_{k}\left(i_{\mathrm{CELL}}\right)
$$

where

$$
\begin{aligned}
& V_{\mathrm{PE}}\left(i_{\mathrm{CELL}}\right)=V_{\mathrm{OC}}+\int_{0}^{i_{\mathrm{CELL}}} R_{\mathrm{PE}}(i) d i \\
& V_{\mathrm{k}}\left(i_{\mathrm{CELL}}\right)=\int_{0}^{i_{\mathrm{CEL}}} R_{\mathrm{k}}(i) d i
\end{aligned}
$$

The model is constructed by deriving analytical expressions for the partial IV curves in Equation 16, from which the corresponding current dependent resistances are obtained by differentiation (cf. Equation 11). Note that deriving the IV characteristics gives us directly the complete IV model. The theoretical expressions for the resistances are needed only for the estimation of the model parameters by EIS.

To arrive at an analytical solution we make two further assumptions. Firstly, the substrate mediated electron recombination is assumed to be negligible compared to the recombination taking place within the porous $\mathrm{TiO}_{2}$ film. This is usually the case at normal light intensities, and also when recombination blocking layer is employed at the substrate even at low light intensities. ${ }^{[30]}$ In practice, $R_{\mathrm{SU}}$ is considered infinite, with the result that all photocurrent 
entering the substrate contact from the $\mathrm{TiO}_{2}$ film is measurable in the external circuit connected to the cell

$$
i_{\mathrm{CELL}}=i_{\mathrm{TiO} 2}
$$

Secondly, we assume that the contact between the porous photoelectrode film and its substrate has negligible resistance $\left(R_{\mathrm{CO}}=0\right)$, meaning that we neglect the $R_{\mathrm{CO}}-C_{\mathrm{CO}}$ pair in the model (Figure 6a). The effect of $R_{\mathrm{CO}}$ has been detected only in the case of certain recombination blocking layers ${ }^{[30]}$ and sometimes when ITO is used instead of FTO at the substrate. ${ }^{[30,37]}$ Although it would straightforward to add the effect of $R_{\mathrm{CO}}$ to the present device model, we omit it here in the absence of an establish physical model for it. From this assumption, it follows that $V_{\mathrm{CO}}=0$ and $V_{\mathrm{PE}}=V_{\mathrm{TiO} 2}$ at all cell current densities (Figure 6b). The photoelectrode voltage is therefore denoted with $V_{\mathrm{TiO} 2}$ in the rest of the paper.

Note finally that voltages that we aim to model in Equations $16-18$ are by their nature purely electrical potential differences across the cell components and their interfaces. Although chemical potential differences (charge carrier concentration profiles) are present both in the semiconductor photoelectrode and in the electrolyte, and are included in the modeling, they do not appear explicitly in the final expressions of the model.

\section{Photocurrent generation}

The $i_{\mathrm{SC}}$ of DSC photoelectrode under white light illumination is determined by the overlap between its spectral incident-photon-to-collected-electron efficiency (IPCE) $\eta_{\mathrm{IPCE}}(\lambda)$ and the spectral photon flux $\Phi_{\mathrm{DC}}(\lambda)$ (units $\mathrm{m}^{-2} \mathrm{~s}^{-1} \mathrm{~nm}^{-1}$ ) incident on the cell

$$
i_{\mathrm{SC}, \mathrm{TiO} 2}=q_{\mathrm{e}} \int_{\lambda_{\min }}^{\lambda_{\max }} \Phi(\lambda) \eta_{\mathrm{IPCE}}(\lambda) d \lambda
$$

where $\lambda_{\min } \ldots \lambda_{\max }$ defines a wavelength range where both of them are non-zero. The IPCE of DSC is determined by three factors: the light harvesting efficiency $\left(\eta_{\mathrm{LH}}\right)$, the electron 
injection efficiency $\left(\eta_{\mathrm{INJ}}\right)$, and the electron collection efficiency $\left(\eta_{\mathrm{COL}}\right)$, which all may be generally wavelength-dependent (Figure 7). ${ }^{[38]}$

$$
\eta_{\mathrm{IPCE}}(\lambda)=\eta_{\mathrm{LH}}(\lambda) \eta_{\mathrm{INJ}}(\lambda) \eta_{\mathrm{COL}}(\lambda)
$$

Note that in a low performance DSC $i_{\mathrm{SC}}$ may be limited by the voltage losses in other cell components and hence be smaller than the photocurrent given by Equation 20. For example mass transport in the electrolyte may set an upper limit to $i_{\mathrm{SC}}$ as described in Section 5.1.

The IPCE can be measured simply by recording the steady state $i_{\text {SC }}$ produced by a calibrated, typically low intensity, monochromatic photon flux directed on the cell while scanning the wavelength of the light with a monochromator. Alternatively, the differential IPCE in the presence of high intensity white bias light can be measured by modulating the monochromatic light beam with a chopper and recording the amplitude of the corresponding $i_{\mathrm{SC}}$ signal with a lock-in amplifier. In practice, DSCs often exhibit non-linear $\eta_{\mathrm{IPCE}} \mathrm{Vs}$. light intensity due to mass transport limitations in the electrolyte or electron concentration dependence of the electron diffusion length, ${ }^{[38-41]}$ and slow photocurrent response time due to electron trapping, ${ }^{[39]}$ which complicate respectively the steady state and differential IPCE measurements and their interpretation. ${ }^{[39,41]}$ This should be kept in mind when using the measured data to estimate $i_{\mathrm{SC}}$ with Equation 20.

\subsection{Light harvesting efficiency}

For the light harvesting efficiency, we use our earlier simplified optical model of DSC. ${ }^{[38,42]}$ When the light is incident on the cell from the photoelectrode side (PE illumination) the $\eta_{\mathrm{LH}}$ is

$$
\eta_{\mathrm{LH}, \mathrm{PE}}=T_{\mathrm{TCO}}\left[1-r_{\mathrm{PE}}\right] \frac{\alpha_{\mathrm{D}}}{\alpha}\left(1-e^{-\alpha d}\right)
$$

and in the case of the opposite CE illumination it is 


$$
\eta_{\mathrm{LH}, \mathrm{CE}}=T_{\mathrm{CE}} T_{\mathrm{EL}}\left[1-r_{\mathrm{PE}}\right] \frac{\alpha_{\mathrm{D}}}{\alpha}\left(1-e^{-\alpha d}\right)
$$

In the above equations, $T_{\mathrm{TCO}}, T_{\mathrm{CE}}$ and $T_{\mathrm{EL}}$ are the transmittance of the TCO-coated glass substrate of the photoelectrode, the counter electrode and the free electrolyte layer respectively, $r_{\mathrm{PE}}$ is the reflectance of the photoelectrode film, and $d$ is the photoelectrode film thickness. The absorption coefficient of the electrolyte filled dyed photoelectrode film is

$$
\alpha(\lambda)=\alpha_{\mathrm{D}}(\lambda)+P \varepsilon(\lambda) \alpha_{\mathrm{EL}}(\lambda)
$$

where $\alpha_{\mathrm{EL}}(\lambda)$ is the absorption coefficient of the bulk electrolyte solution, and $P$ is the porosity of the film. Light scattering is taken into account by an average optical mean path length parameter $\varepsilon$ taking values $1 \leq \varepsilon \leq 2^{[43]}$, and implicitly included in the value of the effective absorption coefficient $\alpha_{\mathrm{D}}$ of the dyed photoelectrode film in the absence of the light absorbing species in the electrolyte. The transmittance of the bulk electrolyte layer is simply

$$
T_{\mathrm{EL}}=e^{-\alpha_{\mathrm{EL}} d_{\mathrm{EL}}}
$$

where $d_{\mathrm{EL}}$ is the thickness of the bulk electrolyte layer.

Due to the thick multilayered solar cell structure (Figure 8) and light scattering by the photoelectrode film, optical characterization of DSC is done one cell component at a time using optical sample cells where the measured component, e.g. the $\mathrm{TiO}_{2}$ photoelectrode film, is prepared on thin plain glass substrate, faced with another glass substrate and wetted with the same solvent as used in the solar cell. ${ }^{[38,44]}$ This minimizes the loss of scattered light in the integrating sphere measurements that are needed to obtain quantitative optical data ${ }^{[44,45]}$ and accounts for the solvatochromism of the dye. ${ }^{[46]}$

\subsection{Electron injection efficiency}

A physical model for the electron injection efficiency could in principle be derived from the fundamentals of electron transfer kinetics, ${ }^{[47]}$ but this is out of the scope of this paper. In practice, the $\eta_{\mathrm{INJ}}$, that may be wavelength dependent, can be determined experimentally by 
various kinetic techniques such as ultra-fast transient absorption spectroscopy, ${ }^{[48,49]}$ timeresolved single-photon counting ${ }^{[50-52]}$ or time-resolved photoacoustic calorimetry. ${ }^{[53]}$ Alternatively, the spectral $\eta_{\mathrm{INJ}}$ can be determined by careful but straightforward combination of readily available optical characterization and steady state IPCE-ratio measurements ${ }^{\text {[38] }}$ (Equations 31 and 32 below) that have shown to yield data that is consistent with the kinetic techniques. ${ }^{[51]}$ For a given DSC, the $\eta_{\mathrm{INJ}}$ may be assumed to be constant that is independent on the photoelectrode voltage ${ }^{[52]}$ and position in the photoelectrode film, but can be significantly affected by surface charge in the $\mathrm{TiO}_{2}$ as determined by the electrolyte composition and molecular structure of the $\mathrm{TiO}_{2}-$ dye - electrolyte interface. ${ }^{[38,52]}$ In the experimental demonstration of the model example in Section $7, \eta_{\mathrm{INJ}}$ is assumed to independent of light wavelength.

\subsection{Electron collection efficiency}

The electron collection efficiency can be described by the standard diffusion model of electron generation, transport and recombination at the nanostructured photoelectrode introduced originally for DSCs by Södergren et al.. ${ }^{[33]}$

The diffusion model is based on solving the continuity equation for the free electron density $n$ assuming that the electron recombination is linear with $n$

$$
\frac{\partial n}{\partial t}=D \frac{\partial^{2} n}{\partial x^{2}}-\frac{n-n_{0}}{\tau}+g
$$

where $D$ and $\tau$ are the electron diffusion coefficient and lifetime, respectively, $g=g(x, t)$ is the electron generation rate, and $n_{0}$ is the equilibrium electron concentration in the dark. The generation terms that are consistent with our optical model (Equations 22a-b) and steady state conditions are

$$
g_{\mathrm{PE}}(x)=\Phi \eta_{\mathrm{INJ}} T_{\mathrm{TCO}}\left[1-r_{\mathrm{PE}}\right] \alpha_{\mathrm{D}} e^{-\alpha x}
$$




$$
g_{\mathrm{CE}}(x)=\Phi \eta_{\mathrm{INJ}} T_{\mathrm{CE}} T_{\mathrm{EL}}\left[1-r_{\mathrm{PE}}\right] \alpha_{\mathrm{D}} e^{\alpha(x-d)}
$$

The model is solved by applying a voltage dependent boundary condition at the photoelectrode substrate contact and a reflecting boundary condition at the opposite edge of the film

$$
\begin{aligned}
& n(x=0)=n_{0} e^{\frac{E_{\mathrm{F}}(x=0)-E_{\text {rdox }}(x=d)}{m k_{\mathrm{B}} T}}=n_{0} e^{-\frac{q_{e} V_{\mathrm{TiO}}}{m k_{\mathrm{B}} T}} \\
& \left.\frac{\partial n}{\partial x}\right|_{x=d}=0
\end{aligned}
$$

where $V_{\mathrm{TiO} 2}$ is the photoelectrode voltage that corresponds to the difference between Fermi level in the $\mathrm{TiO}_{2} E_{\mathrm{F}}$ and redox potential in the electrolyte $E_{\text {redox. }}$ In Equation 27 an empirical ideality factor $m$ (a.k.a. nonideality factor) is introduced. ${ }^{[33]}$ The ideality factor is needed to account for the failure of the "ideal" model $(m=1)$ to explain certain experimental characteristics of the DSC photoelectrode function, such as the light intensity dependence of

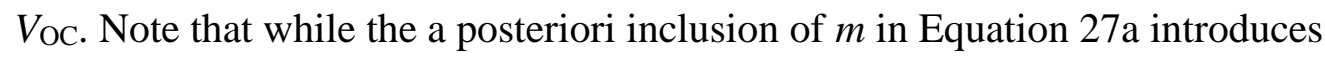
physical inconsistency in the model, as pointed out by Bisquert and Mora-Seró ${ }^{[54]}$, it is necessary here to reach an analytical solution. Moreover, the physical origin of the nonideality is not yet well established (see discussion in Section 4.2 below). We therefore adhere to the conventional linear model here and treat $m$ as an empirical parameter that needs to be determined by experiments for each type of DSC under study.

The solution for the steady state electron collection efficiency is obtained at the short circuit condition of the photoelectrode, i.e. $V_{\mathrm{TiO} 2}=0$ in Equation 27 with the following results ${ }^{[33,38]}$

$$
\eta_{\mathrm{COL}, \mathrm{PE}}=\frac{\left[-L \alpha \cosh (d / L)+\sinh (d / L)+L \alpha e^{-\alpha d}\right] L \alpha}{\left(1-e^{-\alpha d}\right) \cdot\left[1-L^{2} \alpha^{2}\right] \cosh (d / L)}
$$

and for the CE illumination

$$
\eta_{\mathrm{COL}, \mathrm{CE}}=\frac{\left[L \alpha \cosh (d / L)+\sinh (d / L)-L \alpha e^{\alpha d}\right] L \alpha e^{-\alpha d}}{\left(1-e^{-\alpha d}\right) \cdot\left[1-L^{2} \alpha^{2}\right] \cosh (d / L)}
$$


where $L=(D \tau)^{1 / 2}$ is the electron diffusion length.

More generally, if the optical properties of the solar cell are such that the spatially varying electron generation rate $g(x)$ is not exponential like in Equations 26a and 26b, the collection efficiency can be calculated from the following equation ${ }^{[42]}$

$$
\eta_{\mathrm{COL}}=\frac{\int_{0}^{d} g(x) \eta_{\mathrm{COL}, \delta}(x) d x}{\int_{0}^{d} g(x) d x}
$$

where $\eta_{\mathrm{COL}, \delta}$ is the collection efficiency of electrons injected into the film at $x=\delta$, also known as the spatial electron collection efficiency, ${ }^{[42]}$ given by

$$
\eta_{\mathrm{COL}, \delta}(x)=\frac{\cosh \left(\frac{d-x}{L}\right)}{\cosh \left(\frac{d}{L}\right)}
$$

Equation 30 tells us that the probability that a photogenerated electron reaches the collecting substrate contact before it is lost by recombination is the higher the closer to the contact it is generated (Figure 9). The overall collection efficiency thus depends on the average distance of electron generation from the collecting contact compared to the electron diffusion length. Experimental evaluation of the electron diffusion length and the collection efficiency in DSC is problematic and presently under keen investigations. The dominant method has been based on measurement of the electron diffusion coefficient and lifetime by dynamic photocurrent and photovoltage techniques, such as the IMPS ${ }^{[55,56]}$ and IMVS, ${ }^{[56-58]}$ or related time-domain transient measurements. ${ }^{[56,59-64]}$ However, these dynamic techniques have been recently called into question. ${ }^{[65-67]}$ It became first evident that the dynamic data are significantly affected by electron trapping which does not necessarily pay any relevance to the steady state operation of the cell, ${ }^{[65]}$ and later it was shown that the dynamic techniques yield $L$ estimates that are too large to be consistent with measured steady state photocurrent density ${ }^{[51,66]}$ or trapped 
electron density. ${ }^{[67]}$ Hence, there is a need for practical and accurate steady state methods for electron collection measurements. One such method, based on the analysis of steady state IPCE spectra, was proposed already by Södergren et al.. ${ }^{[33]}$ Recently, this technique was brought to a more quantitative basis in the form of an IPCE-ratio method that allows not only determination of the electron collection efficiency but also the electron injection efficiency, by combination of IPCE and optical measurements. ${ }^{[38]}$ The method is based on the fact that the ratio of IPCE spectra of a semitransparent DSC measured from the opposite directions of illumination is sensitive to the electron diffusion length when roughly $L<3 d$, and independent of the electron injection efficiency. ${ }^{[38]}$ Hence, combining Equations 21d, 22a-b, we can express the ratio of the electron collection efficiencies as

$$
\frac{\eta_{\mathrm{COL}, \mathrm{CE}}(L, d, \alpha(\lambda))}{\eta_{\mathrm{COL}, \mathrm{PE}}(L, d, \alpha(\lambda))}=\frac{T_{\mathrm{TCO}}(\lambda)}{T_{\mathrm{CE}}(\lambda) T_{\mathrm{EL}}(\lambda)} \cdot \frac{\eta_{\mathrm{IPCE}, \mathrm{CE}}(\lambda)}{\eta_{\mathrm{IPCE}, \mathrm{PE}}(\lambda)}
$$

where an expression for the left hand side is readily obtained from Equations 28a-b. In this equation, $d, \alpha$, the IPCE-ratio, and the optical transmittances can be measured independently by IPCE and optical techniques leaving $L$ as the only unknown that can be thus determined, treating it either as a constant ${ }^{[51,66]}$ or a wavelength dependent variable. ${ }^{[38]}$ Once $L$ is determined, the injection efficiency can be calculated as

$$
\eta_{\mathrm{INJ}}(\lambda)=\frac{\eta_{\mathrm{IPCE}, \mathrm{PE}}(\lambda)}{\eta_{\mathrm{LH}, \mathrm{PE}}(\lambda, d) \eta_{\mathrm{COL}, \mathrm{PE}}(L, d, \alpha(\lambda))}
$$

with the measured $\eta_{\mathrm{IPCE}}$, optically determined $\eta_{\mathrm{LH}}$, and $\eta_{\mathrm{COL}}$ calculated from Equation $28 \mathrm{a}$, using data from PE illumination (as in the above expression) or CE illumination, both giving the same result by definition. Note that for the determination of $\eta_{\mathrm{COL}}$ this method works only when electron collection losses are significant, i.e. when the method is most needed in practice, and that the accuracy of the $\eta_{\mathrm{INJ}}$ estimates is sensitive to the absolute calibration of the IPCE measurement system and the accuracy of the estimation of $\eta_{\mathrm{LH}}$ by the optical characterization and modeling. Also, it was very recently shown by Villanueva-Cab et al. ${ }^{[68]}$ 
that the IPCE-ratio method is not correct if the electron recombination is not first-order in the free electron concentration, as is assumed in Equations 28a and 28b used in Equation 31. This means that the search for an accurate method to analyze recombination losses at the short circuit condition continues.

\subsection{Typical spectral photocurrent characteristics}

Figure 10a-b shows typical optical performance data corresponding to Equations 22a-b. The light absorption by the N719 dye used in this case extents to ca. $820 \mathrm{~nm}$ and is close to $100 \%$ for this film thickness below $600 \mathrm{~nm}$. The transmittance of the FTO glass substrate of the photoelectrode is ca. $80-85 \%$ without an antireflection coating and is reduced by ca. $4-$ $5 \%$-units for the counter electrode due to light absorption by the platinum catalyst layer. The light absorption by the electrolyte is due to tri-iodide, and it is significant up to $650 \mathrm{~nm}$ and particularly strong below $520 \mathrm{~nm}$. This causes a marked reduction of the $\eta_{\mathrm{LH}}$ at the CE side illumination compared to normal case when light is incident on the cell from the PE direction (Figure 10b). The side of illumination has an effect also on $\eta_{\mathrm{COL}}$. With the PE illumination, the electron collection is ca. $95 \%$ efficient at strongly absorbed light wavelengths below 550 $\mathrm{nm}$ and decreases towards longer wavelengths, whereas at the $\mathrm{CE}$ illumination, $\eta_{\mathrm{COL}}$ is ca. $9 \%$-units lower below $550 \mathrm{~nm}$, but increases towards longer wavelengths. This shows that even though the present electron diffusion length $(L=21.8 \mu \mathrm{m})$ is longer than the film thickness $(d=12.8 \mu \mathrm{m})$ significant electron collection losses still arise. Whenever this is the case, the $\eta_{\mathrm{COL}}$ is sensitive to the direction of illumination and light wavelength that both determine the electron generation profile, and thus the average distance the electrons have to travel to the collecting contact. At the most weakly absorbed wavelengths, the generation profile is almost flat and $\eta_{\mathrm{COL}}$ is close to equal from both directions (Figure 10b). 


\section{Photoelectrode resistance and IV curve}

Having set a model for the photocurrent generation in DSC, we now turn to the main topic of the paper: modeling of the IV curve and its differential resistance. This is determined in the first place by the dye-sensitized nanostructured photoelectrode. Here we use the model of Södergren et al., ${ }^{[33]}$ which is based on solving the above mentioned diffusion model with boundary conditions $27 \mathrm{a}-\mathrm{b}$ and calculating the current density at the substrate contact as

$$
i_{\mathrm{CELL}}=\left.q_{\mathrm{e}} D \frac{d n}{d x}\right|_{x=0}
$$

in accordance with the electron diffusion. Note that we neglect direct electron recombination via the photoelectrode substrate, i.e. $i_{\mathrm{CELL}}=i_{\mathrm{TiO} 2}$ as mentioned before (cf. Figure $6 \mathrm{~b}$ ).

The solution, when adapted from the expression given by Södergren et al. ${ }^{[33]}$ to the present case, is

$$
i_{\mathrm{CELL}}\left(V_{\mathrm{TiO} 2}\right)=i_{\mathrm{SC}, \mathrm{TiO} 2}-\frac{q_{\mathrm{e}} D n_{0}}{L} \tanh \left(\frac{d}{L}\right)\left(e^{-\frac{q_{e} V_{\mathrm{TiO} 2}}{m k_{\mathrm{B}} T}}-1\right)
$$

where $i_{\mathrm{SC}, \mathrm{TiO} 2}$ is the short circuit current density of the photoelectrode (Equation 20c).

Equivalent result was obtained for the dark conditions $\left(i_{\mathrm{SC}, \mathrm{TiO} 2}=0\right)$ by Bisquert. ${ }^{[69]}$

Equation 34 can be simplified by considering the voltage dependence of the electron recombination reaction. According to the first order recombination kinetics implicit in Equation 25, the recombination current density flowing through a photoelectrode film of thickness $d$ and uniform electron density $n$ is

$$
i_{\mathrm{REC}}=q_{\mathrm{e}} d k\left(n-n_{0}\right)=q_{\mathrm{e}} d \frac{n-n_{0}}{\tau}
$$


where $k$ is the first order rate constant of the recombination reaction, and $\tau$ the corresponding electron lifetime which is assumed to be independent of the position in the film. Inserting the basic relation between electron density and voltage (Equation 27a) to Equation 35 we get

$$
i_{\mathrm{REC}}\left(V_{\mathrm{TiO} 2}\right)=i_{\mathrm{REC}, 0}\left(e^{-\frac{q_{\mathrm{e}} V_{\mathrm{TiO} 2}}{m k_{\mathrm{B}} T}}-1\right)
$$

where

$$
i_{\mathrm{REC}, 0}=\frac{q_{\mathrm{e}} d n_{0}}{\tau}
$$

may be called the exchange current density of the recombination reaction. The differential resistance of Equation 34 is called the electron recombination resistance $R_{\mathrm{REC}}\left(\Omega \mathrm{cm}^{-2}\right)$. It depends on the voltage as

$$
R_{\mathrm{REC}}\left(V_{\mathrm{TiO} 2}\right)=\left(\frac{d i_{\mathrm{CELL}}}{d V_{\mathrm{TiO} 2}}\right)^{-1}=\left(\frac{-d i_{\mathrm{REC}}}{d V_{\mathrm{TiO} 2}}\right)^{-1}=R_{\mathrm{REC}, 0} e^{\frac{q_{\mathrm{e}} V_{\mathrm{TiO} 2}}{m k_{\mathrm{B}} T}}
$$

and on the current as

$$
R_{\mathrm{REC}}\left(i_{\mathrm{CELL}}\right)=R_{\mathrm{REC}, 0}\left(1-\frac{i_{\mathrm{CELL}}}{i_{\mathrm{REC}, 0}}\right)^{-1}
$$

where

$$
R_{\mathrm{REC}, 0}=\frac{m k_{\mathrm{B}} T}{q_{\mathrm{e}} i_{\mathrm{REC}, 0}}
$$

is the recombination resistance at zero voltage $\left(V_{\mathrm{TiO} 2}=0\right)$, and we have used the conditions $i_{\mathrm{CELL}}=i_{\mathrm{REC}}$ and $i_{\mathrm{SU}}=0$ (cf. Figure $\left.6 \mathrm{~b}\right)$.

Note that Equation 36 is very similar to the second term in Equation 34, which can be written as

$$
i_{\mathrm{CELL}}\left(V_{\mathrm{TiO} 2}\right)=i_{\mathrm{SC}, \mathrm{TiO} 2}-i_{\mathrm{REC}, 0}\left(e^{-\frac{q_{\mathrm{e}} V_{\mathrm{TiO} 2}}{m k_{\mathrm{B}} T}}-1\right) \frac{\tanh (d / L)}{d / L}
$$


when we use Equation 37 and recall that $L=(D \tau)^{-1 / 2}$. The only difference is that the recombination current term in Equation 36 is multiplied by the factor $\tanh (d / L) /(d / L)$. Just for the record, this factor is equal to the electron collection efficiency at short circuit for uniform electron generation (cf. Equations 24 and 34 in ref. ${ }^{[42]}$ with $\omega=0$ ). Equation 41 means that both the photocurrent and the recombination current depend on the electron diffusion length. This makes sense, since both are related to transport and recombination of electrons in the film. However, unlike the photocurrent, the recombination current does not depend on the electron generation (light absorption profile etc.) but is purely an electrical property controlled by the potential at the contact. Accordingly, Equation 41 obeys the well known principle of superposition: photocurrent generation displays the IV curve of the photoelectrode along the current axis by a constant, voltage independent amount $i_{\mathrm{SC}, \mathrm{TiO} 2}$.

Solving for $V_{\mathrm{TiO} 2}$ in Equation 41 gives

$$
V_{\mathrm{TiO} 2}\left(i_{\mathrm{CELL}}\right)=-\frac{m k_{\mathrm{B}} T}{q_{\mathrm{e}}} \ln \left(\frac{d}{L} \operatorname{coth}\left(\frac{d}{L}\right) \cdot \frac{i_{\mathrm{SC}, \mathrm{TiO} 2}-i_{\mathrm{CELL}}}{i_{\mathrm{REC}, 0}}+1\right)
$$

which gives directly the equation for the $V_{\mathrm{OC}}$ of the cell, when $i_{\mathrm{CELL}}=0$

$$
V_{\mathrm{OC}}=-\frac{m k_{\mathrm{B}} T}{q_{\mathrm{e}}} \ln \left(\frac{d}{L} \operatorname{coth}\left(\frac{d}{L}\right) \cdot \frac{i_{\mathrm{SC}, \mathrm{TiO} 2}}{i_{\mathrm{REC}, 0}}+1\right)
$$

The differential resistance of the photoelectrode IV curve (Equation 41) is the total resistance of the photoelectrode film $R_{\mathrm{TiO} 2}$, including both electron diffusion and recombination in the film

$$
R_{\mathrm{T} 1 \mathrm{O} 2}\left(V_{\mathrm{TiO} 2}\right)=\left(\frac{d i_{\mathrm{CELL}}}{d V_{\mathrm{TiO} 2}}\right)^{-1}=R_{\mathrm{REC}}\left(V_{\mathrm{TiO} 2}\right)\left[\frac{d}{L} \operatorname{coth}\left(\frac{d}{L}\right)\right]
$$

where $R_{\mathrm{REC}}\left(V_{\mathrm{TiO} 2}\right)$ is given by Equation 38 . Note that Equation 44 is equal to the voltage dependent differential DC resistance of the film in the dark. ${ }^{[69]}$ Equation 42 gives it as a function of cell current density 


$$
R_{\mathrm{TiO} 2}\left(i_{\mathrm{CELL}}\right)=R_{\mathrm{REC}, 0}\left(\frac{i_{\mathrm{SC}, \mathrm{TiO} 2}-i_{\mathrm{CELL}}}{i_{\mathrm{REC}, 0}}+\frac{\tanh (d / L)}{d / L}\right)^{-1}
$$

This equation may be compared with Equation 39 by noting that $i_{\mathrm{SC}, \mathrm{TiO} 2}-i_{\mathrm{CELL}}=i_{\mathrm{REC}}$ : it reduces to Equation 39 when $L \gg d$. This corresponds to a situation where electron diffusion is much faster than their recombination, and hence, the electron density is uniform in the photoelectrode film. Indeed, this was assumed in the derivation of Equation 39.

\subsection{Relation between the diffusion model and the transmission line impedance model}

The total differential resistance of the photoelectrode (Equation 44) that was derived above based on the standard electron diffusion model can also be obtained from the transmission line impedance model summarized in Appendix A (Equation A7). Indeed, it has been shown

that the diffusion model is mathematically equivalent to the transmission line model. ${ }^{[69,70]}$ Here, this equivalence can be seen by comparing Equations 44 and A7 while acknowledging that electron diffusion coefficient $D$, lifetime $\tau$ and diffusion length $L$ are related to the electron transport and recombination resistance via the chemical capacitance $C_{\mu}$ per unit area of the electrode $\left(\mathrm{F} \mathrm{m}^{-2}\right)$ as ${ }^{[36,70-72]}$

$$
\begin{aligned}
& D=\frac{d^{2}}{R_{\mathrm{T}} C_{\mu}} \\
& \tau=R_{\mathrm{REC}} C_{\mu} \\
& L=\sqrt{D \tau}=d \sqrt{\frac{R_{\mathrm{REC}}}{R_{\mathrm{T}}}}
\end{aligned}
$$

In practice, $L$ can be determined from an impedance spectrum that displays both the electron recombination arc $\left(R_{\mathrm{REC}}\right)$ and the linear transport part $\left(1 / 3 R_{\mathrm{T}}\right)$ (Figure 11) by fitting the transmission line model (Equation A1 in the Appendix) to the data and using Equation 48. This means that our photoelectrode resistance model can be verified and its parameters estimated by EIS measurements (Figure 11) using the above relations. However, the two 
models are not physically equivalent. ${ }^{[70]}$ In fact, the diffusion model gives more correct description of the DSC operation than the transmission line model, which can be understood by comparing the assumptions behind the models.

The diffusion model assumes that the electron lifetime is constant whereas the electron density may vary with position in the film. This means that the Fermi level (Equation 27a) and thus the electron recombination resistance (Equation 38) are spatially varying. This contradicts the basic assumption in the transmission line model that the distributed circuit elements, including the recombination resistance, are constant. Hence, the equivalence of Equations 44 to A7 is merely a mathematical fact that has physical meaning only if we assign in the transmission line model the distributed recombination resistance a constant value $r_{\mathrm{REC}}\left(V_{\mathrm{TiO} 2}\right)=R_{\mathrm{REC}}\left(V_{\mathrm{TiO} 2}\right) d^{-1}$ that is determined by the voltage $V_{\mathrm{TiO} 2}$ at the substrate contact. However, as already mentioned a constant $r_{\mathrm{REC}}$ is against the basic characteristics of the diffusion model.

It is well known from experiments that the electron density is not uniform but attains a profile in the photoelectrode of an operating DSC, ${ }^{[34,67,73,74]}$ and that the recombination resistance depends exponentially on the voltage (Fermi level). ${ }^{[26,28,31,67,72]}$ These effects are captured by the diffusion model, at least qualitatively, but not at all by the transmission line model. Hence, although the diffusion model has itself many shortcomings that are discussed in the following sections, we have to conclude for the time being that it is a better physical model for DSC than the transmission line model. Nevertheless, the transmission line model provides us with a mathematical tool to interpret and analyze the EIS data and estimate the diffusion model parameters. It should be kept in mind however, that the physical mismatch of the models causes errors in the estimated values of $D$ and $\tau$, or $r_{\mathrm{T}}$, and $r_{\mathrm{REC}}$, these errors being the larger the less uniform the electron concentration is in the conditions of the EIS measurements, ${ }^{[54,70]}$ favorable conditions being open circuit state under illumination and whenever $L \gg d$. Note however, the total resistances $R_{\mathrm{T}}$ and $R_{\mathrm{REC}}$ are most likely always valid estimates of the 
overall contributions from electron transport and recombination to the total differential resistance of the photoelectrode, $R_{\mathrm{TiO}}$, that is always correctly measured with EIS because of its direct relation to the IV curve (Equation 11).

\subsection{Interpretation of the ideality factor}

The ideality factor $m$ introduced to Equation $27 \mathrm{a}$ is the only parameter in our device model that does not have a clear physical interpretation. However, it is needed in the model to account for the experimentally observed nonideal characteristics of DSCs. The nonideality can be seen in Figure 12, which at the same time demonstrates three different experimental methods for estimating $m$. Note that the three methods yield quite different $m$ values.

However, there are reasons to consider the value $m=2.1$ derived from current dependent $R_{\mathrm{REC}}$ analysis (Figure 12a) as the most reliable estimate. Firstly, analysis based on the cell voltage (Figure 12b) under the assumption that $V_{\mathrm{CELL}}=V_{\mathrm{TiO} 2}$ is biased by the voltage losses in the other cell components, leading to too high $m$ values, here $m=2.5$. The error could be corrected by evaluating the voltage losses from the impedance data (Equations 16 and 18, cf. refs., $\left.{ }^{[28,31]}\right)$, but would require extra work that is unnecessary with the simple current based analysis. Figure 12c demonstrates the more traditional method based on light intensity dependence of $V_{\mathrm{OC}}$ analyzed with Equations 43 and 20, giving even higher nonideality with $m$ $=2.8$. The source for the error in the present data is not clear, but in general, this method is known to be affected by additional electron recombination via the substrate - electrolyte interface, and possibly by secondary light-induced effects, as discussed below.

Different hypothesis on the origin of the nonideality have been put forward in the literature. Electron recombination via the photoelectrode substrate - electrolyte interface has been shown to significantly increase the nonideality, measured by the light intensity dependence of $V_{\mathrm{OC}}$, inasmuch as application of a compact recombination blocking layer between the TCO layer and the nanostructured $\mathrm{TiO}_{2}$ film has significantly decreased increased the ideality (i.e. 
reduced the value of $m$ closer to 1$).{ }^{[30,75-78]}$ However, close to ideal behavior seems to be observed only rarely, e.g. ${ }^{[3,79]}$, typical values of $m$ being instead in the range $m=1.25-1.9$ even with a blocking layer ${ }^{[30,75,78]}$ as well as in the case of Ti metal substrates $(m=1.6-$ 1.9). ${ }^{[80,81]}$ This suggests that the nonideality is a property of the photoelectrode film and not merely a substrate effect. As pointed out by Bisquert and Mora-Seró,${ }^{[54]}$ the same conclusion can also be made based on the nonideality of $R_{\text {REC }}$ (Figure 12a-b) that is dominated by the photoelectrode film at high $V_{\text {CELL }}$ and $i_{\text {CELL }}$ in the dark (whereas at low $V_{\text {CELL }}$ and $i_{\text {CELL }}$ it is affected by $\left.R_{\mathrm{SU}}\right)^{[26,30]}$

An early hypothesis for the nonideality was electron recombination kinetics that are higher order in the conduction band electrons density, ${ }^{[40,82]}$ i.e. the reaction order in electron density being $m^{-1}$ in light of Equations 27a and 25. However, there are variable experimental evidence regarding the reaction mechanism, most recently the results of Wang and Peter giving support to the first order recombination kinetics. ${ }^{[67]}$ According to another hypothesis, the nonideal characteristics are related to electron recombination via surface states distributed in exponentially energy in the band gap of the $\mathrm{TiO}_{2} \cdot{ }^{[31,40,72,80]}$ Indeed, Bisquert et al. ${ }^{[31,80]}$ have shown that if the electron recombination occurs predominantly via such states, and the occupancy of these states is determined by the Fermi level in the bulk $\mathrm{TiO}_{2}$, the voltage dependence of the recombination resistance takes the same form as in Equation 38, but with the ideality factor being replaced by $m=(1 / 2+\alpha \mathrm{S})^{-1}$, where $\alpha_{\mathrm{S}}$ is the surface state distribution parameter. Hence, this model provides a microscopic explanation for the nonideality of the recombination resistance (Equation 38) and the photoelectrode IV characteristics (Equation 41). However, it has been pointed out by the same authors the mere observation of the nonideal characteristics is not a sufficient proof for the existence of surface state mediated recombination. ${ }^{[72]}$ In fact, Peter et al. have speculated that the nonideality might be a manifestation of nonideal thermodynamics of electrons in the $\mathrm{TiO}_{2}$ described by an electron activity coefficient, ${ }^{[81]}$ a possible source for this being Coulombic interaction of electrons with 
the ions in the electrolyte. ${ }^{[83]}$ Finally, O'Regan and Durrant has presented yet another view in which the nonideality may be related to the failure of the $\mathrm{TiO}_{2}$ conduction band edge energy vs. the redox level in the electrolyte to remain fixed in an operating DSC (an assumption made in Equation 27a), prompted by the observation of a conduction band edge shift with increasing $V_{\mathrm{OC}}$ in their measurements of the activation energy of electron transport and recombination. ${ }^{[84]}$

It becomes apparent that a great deal of microscopic physical details of the DSC photoelectrode function may be embedded in the nonideality, but nevertheless relatively well described by the single ideality constant $m$. It therefore seems for the time being reasonable to stick to the simple nonideal diode model represented by Equations 38 - 41, until the different hypotheses for the origin of the nonideality are scrutinized experimentally. Presently it seems that the hypothesis of Bisquert et al. (non-linear recombination) is best supported by experimental data and and physical modeling ${ }^{[31,54,80]}$. To correctly account for it in the DSC photoelectrode model it should be incorporated already in the continuity equation (Equation 25), which makes the model solvable only numerically. ${ }^{[54]}$ According to Bisquert and MoraSeró, neglegting this by using a linear model instead leads to errors in the interpretation of the measured electron diffusion length ${ }^{[54]}$. However, it remains to be examined how significant these errors are from the point-of-view practical DSC performance characterization. Until this is clarified, the ideality factor may be considered as an additional performance loss factor related to the electron recombination. In fact, as shown by Fabregat-Santiago et al. ${ }^{[28]}$ and in Figure $12 \mathrm{~d}$, for a fixed $V_{\mathrm{OC}}$ the nonideality $(m>1)$ correspond to a voltage loss that decreases the fill factor, and hence, its effect is similar to the internal cell resistances.

\subsection{Influence of electron trapping}

In our photoelectrode model discussed above we take into account only conduction band electrons and neglect the influence of electron trapping. It is generally acknowledged that 
under normal operating conditions most of the electrons in the $\mathrm{TiO}_{2}$ are in fact trapped in localized states in the band gap (Figure 13). However, it has been shown both theoretically ${ }^{[65]}$ and by numerical simulations ${ }^{[85]}$ that electron trapping does not affect the steady state operation of DSC as long as the traps do not offer an additional pathway to electron transport and recombination. Indeed, if recombination via trap states (e.g. surface states) in the band gap is insignificant compared to recombination directly from the conduction band, the trapped and free (conduction band) electron populations are in equilibrium with each other at the open circuit condition, i.e. they are characterized by a common Fermi level. If, in addition to this, direct electron transport between trap states by electron hopping or tunneling mechanisms does not take place, the "quasi-equilibrium" [65] between the trapped and free electrons holds also when there is a net electron flux in the photoelectrode film, i.e. along the whole solar cell IV curve. Hence, it is a valid approximation to adopt the above assumptions and base the steady state DSC performance modeling solely on the conduction band electrons. The possibility of electron recombination via surface traps is then accounted for by the ideality factor $m$ discussed earlier.

This said, it must be pointed out that the trapped electrons do influence the dynamic operation of DSC, since they contribute to the chemical capacitance $C_{\mu}$ of the photoelectrode film. ${ }^{[65]}$ In fact, $C_{\mu}$ is generally assumed to be dominated by the trapped electrons, and this has several consequences. Firstly, it means that the $D$ and $\tau$ derived from dynamic measurements like EIS (Equations 46 and 47) do not represent the electron diffusion coefficient and lifetime of the conduction band electrons used in the diffusion model in Section 3.3. Secondly, since the trapped electrons, and the cell capacitance that they dominate, are irrelevant to the steady state solar cell operation, as mentioned above, the same applies to the dynamically measured values of $D$ and $\tau$ as well. ${ }^{[65]}$ On the contrary, the measured electron transport and recombination resistances, $R_{\mathrm{T}}$ and $R_{\mathrm{REC}}$, and the diffusion length $L$, are steady state parameters that are 
directly related to the IV curve since they constitute part of its differential resistance (Equations 44 and 48). Hence, it is necessary to base the performance analysis of DSC on these quantities instead of $D$ and $\tau$.

From the above discussion, we may conclude that electron traps play a central role in the DSC operation: Reversible electron trapping to bulk states greatly affects the dynamic response of these solar cells and recombination via surface trap states is presently the only quantitative microscopic model that can explain the nonideality of their IV characteristics. It seems that if one whishes to formulate a physical device model that can reproduce both the experimental steady state and dynamic characteristics of DSC with a single set of physical parameters both free (conduction band) and trapped electrons need to be included in the modeling. In this respect, the choice made by Anta et al. ${ }^{[19,85]}$ to base the modeling on the total electron concentration, dominated by trapped electrons, is noteworthy: it appears to capture both the trapping effects and the nonideality in a relatively simple model formulation.

\section{Counter electrode resistance and IV curve}

At the counter electrode of DSC electrons are returned from the external circuit to the electrolyte via a charge transfer reaction at the electrode - electrolyte interface. The progress of this reaction is associated with a voltage loss (overpotential) that decreases the fill factor of the solar cell IV curve. The differential resistance corresponding to the counter electrode IV curve can be divided into two separate contributions: the charge transfer resistance $R_{\mathrm{CE}}$ and the mass transport (diffusion) resistance $R_{\mathrm{D}}$ as indicated in the equivalent circuit model (Figure 6b). In this Section we use basic electrochemistry to derive a kinetic model that predicts the voltage and current dependence of these resistances, thereby giving them a physical interpretation. In particular, our aim is to derive expressions for the current dependence of $R_{\mathrm{CE}}$ and $R_{\mathrm{D}}$ so that the physical parameters behind them could be estimated from current dependent EIS data of complete DSCs. 
In the case of a DSC with iodine based electrolyte the overall charge transfer reaction at the catalyst coated counter electrode is a two electron transfer reaction (Figure 2)

$$
\mathrm{I}_{3}^{-}+2 \mathrm{e}^{-} \rightarrow 3 \mathrm{I}^{-}
$$

This consists of the following partial reactions ${ }^{[15,86,87]}$

$$
\begin{array}{ll}
\left(\mathrm{I}+\mathrm{e}^{-} \leftrightarrow \mathrm{I}^{-}\right) \times 2 & \text { Charge transfer reaction } \\
\mathrm{I}_{2} \leftrightarrow 2 \mathrm{I} & \text { Fast chemical reaction } \\
\mathrm{I}_{3}^{-} \leftrightarrow \mathrm{I}_{2}+\mathrm{I}^{-} & \text {Fast chemical reaction }
\end{array}
$$

The charge transfer reaction involves two fast chemical reactions that are assumed to be in equilibrium, one of which involves dissociation of iodine at the catalyst surface. The charge transfer reaction is the rate determining step with iodide $\mathrm{I}^{-}$as the reduced species and elementary iodine I as the oxidized species. According to the reaction scheme, the current overpotential equation for the overall process (Equation 49) is

$$
\frac{i_{\mathrm{CELL}}}{i_{\mathrm{CE}, 0}}=\frac{C_{\mathrm{I}}}{C_{\mathrm{I}}^{*}} e^{\frac{\beta q_{\mathrm{e}} v_{\mathrm{CE}}}{k_{\mathrm{B}} T}}-\frac{C_{\mathrm{I}^{-}}}{C_{\mathrm{I}^{-}}^{*}} e^{-\frac{(1-\beta) q_{\mathrm{e}} v_{\mathrm{CE}}}{k_{\mathrm{B}} T}}
$$

where $C_{\mathrm{I}}$ and $C_{\mathrm{I}-}\left(\mathrm{mol} \mathrm{dm} \mathrm{dm}^{-3}\right)$ are the concentrations of the iodine and iodide at the immediate vicinity of the electrode surface, $C_{\mathrm{I}}{ }^{*}$ and $C_{\mathrm{I}}{ }^{*}\left(\mathrm{~mol} \mathrm{dm}{ }^{-3}\right)$ their equilibrium concentrations, $\beta$ is the symmetry parameter, and the overpotential is defined here as the potential of the electrode with respect to the potential in the electrolyte next to the electrode surface, $V_{\mathrm{CE}}=\varphi_{\mathrm{EL}}-\varphi_{\mathrm{CE}}$. The exchange current density $i_{\mathrm{CE}, 0}$ of the overall reaction is ${ }^{[88]}$

$$
i_{\mathrm{CE}, 0}=2 F k_{\text {app }}^{0} C_{\mathrm{I}}^{{ }^{[1-\beta / 2]}} C_{\mathrm{I}}^{*[\beta / 2]}
$$

where $k_{\text {app }}^{0}\left(\mathrm{~cm} \mathrm{~s}^{-1}\right)$ is the apparent standard rate constant of the overall reaction, and $F$ is the Faraday's constant. Equation 52 takes into account that Equation 49 incorporates twice the 
same rate determining electron transfer reaction (i.e. $n=2, n^{\prime}=0$ in Equation 3.5.40 in ref. $\left.^{[88]}\right)$. In order to write this equation in terms of the concentration of the ionic species in the free electrolyte, i.e. iodide and tri-iodide, the chemical balance between $\mathrm{I}^{-} \mathrm{I}^{-}$and $\mathrm{I}_{3}{ }^{-}$(Equations $50 \mathrm{~b}$ and 50c) needs to be taken in to account using the law of mass action with an appropriate equilibrium constant $K$

$$
C_{\mathrm{I}}=K C_{\mathrm{I}_{3}^{-}}^{1 / 2} C_{\mathrm{I}^{-}}^{-1 / 2}
$$

Inserting Equation E12 in Equation E11 yields ${ }^{[15]}$

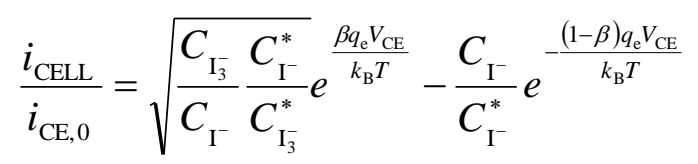

It should be noted that the current overpotential relation (Equation 54) does not include the number of electrons transferred in the overall reaction, $n=2$, in the exponentials. While the overall reaction (Equations 49 - 50c) consists of transfer of two electrons, they are transferred in two identical parallel processes with I and $\mathrm{I}^{-}$respectively as the reduced and oxidized species that are equally available at the electrode surface. Hence, the number of electrons affects the current density via the value of the exchange current density while it responds to the polarization of the electrode - electrolyte interface identically to a single electron transfer process, in analogy to parallel connection of resistances.

Equation 54 with Equation 52 is the complete equation for the IV characteristics of the counter electrode in DSC with the $\mathrm{I}_{3}^{-} / \mathrm{I}^{-}$redox electrolyte, and has been found to be consistent with experiments. ${ }^{[86,87]}$ It shows that the voltage loss $V_{\mathrm{CE}}$ imposed at the counter electrode electrolyte interface by passing a current density $i_{\text {CELL }}$ through it, depends on:

1. Electrocatalytic activity of the electron transfer reaction, i.e. kinetic rate constant $k^{0}$ app (Equation 52) 
2. Equilibrium concentration of the electrolyte species, $C^{*}{ }^{*}$ and $C^{*}{ }^{3-}$ (Equation 52 and 54)

3. Deviation of the surface concentrations $C_{\mathrm{I}^{-}}$and $C_{\mathrm{I} 3}$ - from their equilibrium values due to limited mass transport to and from the electrode surface (Equation 54)

Considering the operation of a given DSC, the exchange current density $i_{\mathrm{CE}, 0}$ at the counter electrode can be taken constant specific to the preparation and materials of the electrolyte and counter electrode $\left(C^{*}{ }^{-}, C^{*}{ }_{13-}\right.$, and $\left.k_{\text {app }}^{0}\right)$. Hence, the voltage loss at the counter electrode in an operating DSC has two contributions: charge transfer and mass transport overpotentials. These contributions are discussed below.

\subsection{Mass transport in the electrolyte}

When DSC is delivering photocurrent to the external circuit, tri-iodide is consumed and iodide is formed at the counter electrode which changes their surface concentrations, and hence, contributes to the voltage loss at the counter electrode. Theoretical analysis of this effect requires setting up an electrolyte mass transport model that gives the relation between the surface concentrations and the current density at the counter electrode. Here, we use the simplest possible mass transport model for DSC: one-dimensional diffusion of electroactive species between two planar electrodes in a thin layer electrochemical cell. In practice this means that we neglect the porosity of the photoelectrode film and assume the charge transfer reactions occur at the photoelectrode substrate instead of over the whole film. This is major simplification of the real situation, that will be discussed later in Section 5.5, but necessary for the present purpose since we aim to a simple analytical model.

Using the Fick's law of diffusion the transport equations for $\mathrm{I}_{3}{ }^{-}$and $\mathrm{I}^{-}$consistent with the electrode reactions (Equation 49) are 


$$
\begin{aligned}
& \frac{1}{2} i_{\mathrm{CELL}}=-F D_{\mathrm{I}^{-}} \frac{d C_{\mathrm{I}_{3}}}{d x} \\
& \frac{3}{2} i_{\mathrm{CELL}}=F D_{\mathrm{I}^{-}} \frac{d C_{\mathrm{I}^{-}}}{d x}
\end{aligned}
$$

From Equations $55 \mathrm{a}$ and $55 \mathrm{~b}$ is follows that the steady state concentration profiles in the electrolyte are linear

$$
\begin{aligned}
& C_{\mathrm{I}_{3}}(x)=C_{\mathrm{I}_{3}^{3}}^{*}-\frac{i_{\mathrm{CELL}}}{2 F D_{\mathrm{I}_{3}^{-}}}\left(x-\frac{d_{\mathrm{EL}}}{2}\right) \\
& C_{\mathrm{I}^{-}}(x)=C_{\mathrm{I}^{-}}^{*}+\frac{3 i_{\mathrm{CELL}}}{2 F D_{\mathrm{I}^{-}}}\left(x-\frac{d_{\mathrm{EL}}}{2}\right)
\end{aligned}
$$

where $x=d_{\mathrm{EL}}$ denotes the electrode where $\mathrm{I}_{3}{ }^{-}$is consumed and $\mathrm{I}^{-}$produced, corresponding to the counter electrode function in operating DSC.

Since the tri-iodide is supplied to the electrode surface by diffusion from the electrolyte, at sufficiently high current densities its surface concentration decreases and starts to limit the charge transfer reaction (Equation 49). This depletion of tri-iodide sets a limit to the maximum current density that the solar cell can deliver. The maximum current limited by the surface concentration of tri-iodide falling to zero at the counter electrode $\left(x=d_{\mathrm{EL}}\right)$ and respectively the iodide concentration falling to zero at the opposite electrode $(x=0)$ is readily obtained from Equations 56a-b as

$$
\begin{aligned}
& i_{\mathrm{lim}, \mathrm{I}_{3}}=\frac{2 F D_{\mathrm{I}_{3}} C_{\mathrm{I}_{3}}^{*}}{\left(d_{\mathrm{EL}} / 2\right)}=\frac{4 F D_{\mathrm{I}_{3}} C_{\mathrm{I}_{3}}^{*}}{d_{\mathrm{EL}}} \\
& i_{\mathrm{lim}, \mathrm{I}}=\frac{\frac{2}{3} F D_{\mathrm{I}^{-}} C_{\mathrm{I}^{-}}^{*}}{\left(d_{\mathrm{EL}} / 2\right)}=\frac{4 F D_{\mathrm{I}_{\mathrm{I}}} C_{\mathrm{I}^{-}}^{*}}{3 d_{\mathrm{EL}}}
\end{aligned}
$$


where $D_{\mathrm{I} 3-}$ is the diffusion coefficient of tri-iodide and $d_{\mathrm{EL}}$ is the distance between the planar electrodes. Note that the above equations do not take into account the electrode porosity (see Section 5.5).

In typical liquid DSC electrolytes the equilibrium concentration of iodide $\left(450 \mathrm{~mol} \mathrm{dm}^{-3}\right)$ is nine times larger than that of tri-iodide $\left(50 \mathrm{~mol} \mathrm{dm}^{-3}\right)$ while their diffusion coefficients may be assumed roughly equal. Equations 57a-b therefore imply that the limiting current density due to iodide diffusion is three times larger than that of tri-iodide, meaning that tri-iodide is the mass transport limiting species in a typical DSC. While this means that there is an excess of iodide in the electrolyte from the point of view of mass transport, a high concentration of iodide is necessary for rapid regeneration of the oxidized dye at the photoelectrode and hence should be maximized. In contrast to this, the tri-iodide concentration is optimal when it is just enough to support the mass transport in the cell: an excess of tri-iodide increases electron recombination at the photoelectrode, as well as optical losses due to light absorption by the tri-iodide in the pores of the photoelectrode film.

The effect of mass transport in the electrolyte is coupled to the kinetic model of Equation 54 by expressing the surface concentrations of tri-iodide and iodide at the counter electrode $(x=$ $\left.d_{\mathrm{EL}}\right)$ as a function of current density (Equations 57a-b)

$$
\begin{aligned}
& \frac{C_{\mathrm{I}_{3}^{-}}\left(x=d_{\mathrm{EL}}\right)}{C_{\mathrm{I}_{3}^{-}}^{*}}=1-\frac{i}{i_{\text {lim }, \mathrm{I}_{3}^{-}}} \\
& \frac{C_{\mathrm{I}^{-}}\left(x=d_{\mathrm{EL}}\right)}{C_{\mathrm{I}^{-}}^{*}}=1+\frac{i}{i_{\text {lim }, \mathrm{I}^{-}}}
\end{aligned}
$$

Inserting these to Equation 54 gives

$$
\frac{i_{\mathrm{CELL}}}{i_{\mathrm{CE}, 0}}=\sqrt{\left(1-\frac{i}{i_{\mathrm{lim}, \mathrm{I}_{3}^{-}}}\right)\left(1+\frac{i}{i_{\mathrm{lim}, \mathrm{I}^{-}}}\right)^{-1}} e^{\frac{\beta q_{\mathrm{e}} V_{\mathrm{CE}}}{k_{\mathrm{B}} T}}-\left(1+\frac{i}{i_{\mathrm{lim}, \mathrm{I}^{-}}}\right) e^{-\frac{(1-\beta) q_{\mathrm{e}} V_{\mathrm{CE}}}{k_{\mathrm{B}} T}}
$$


which provides a one-to-one relation between current density and voltage drop at the DSC counter electrode including both the charge transfer and mass transport kinetics. Our next task is to separate these two contributions and derive expressions for the corresponding charge transfer and the mass transport resistances $R_{\mathrm{CE}}$ and $R_{\mathrm{D}}$.

\subsection{Activation overpotential and charge transfer resistance}

If the ionic diffusion in the electrolyte is assumed fast enough to maintain the surface concentrations of the electroactive species at their equilibrium values (i.e. $i<<i_{\mathrm{lim}}$ ), the current overpotential equation reduces to

$$
\frac{i_{\mathrm{CELL}}}{i_{\mathrm{CE}, 0}}=e^{\frac{\beta q_{\mathrm{e}} V_{\mathrm{CT}, \mathrm{CE}}}{k_{\mathrm{B}} T}}-e^{-\frac{(1-\beta) q_{\mathrm{e}} V_{\mathrm{CT}, \mathrm{CE}}}{k_{\mathrm{B}} T}}
$$

The corresponding overpotential is called activation overpotential $V_{\mathrm{CT}, \mathrm{CE}}$. Equation 60 is also known as the Butler-Volmer equation. The activation overpotential is related to the charge transfer resistance that is labeled $R_{\mathrm{CE}}{ }^{[86]}$

$$
R_{\mathrm{CE}}=\frac{\partial V_{\mathrm{CT}, \mathrm{CE}}}{\partial i_{\mathrm{CELL}}}
$$

Applying this definition to equation 60 gives

$$
R_{\mathrm{CE}}=R_{\mathrm{CT}, 0}\left(\beta e^{\frac{\beta q_{\mathrm{e}} V_{\mathrm{CT}, \mathrm{CE}}}{k_{\mathrm{B}} T}}+(1-\beta) e^{\frac{(1-\beta) q_{\mathrm{e}} V_{\mathrm{CT}, \mathrm{CE}}}{k_{\mathrm{B}} T}}\right)^{-1}
$$

where $R_{\mathrm{CE}, 0}$ is the charge transfer resistance at zero polarization $\left(V_{\mathrm{CT}, \mathrm{CE}}=0\right)$

$$
R_{\mathrm{CT}, 0}=\frac{k_{\mathrm{B}} T}{q_{\mathrm{e}} i_{\mathrm{CE}, 0}}
$$

Note that Equation 60 cannot be solved for $V_{\mathrm{CT}, \mathrm{CE}}$ unless we assume that $\beta=0.5$, in which case 


$$
\frac{i_{\mathrm{CELL}}}{i_{\mathrm{CE}, 0}}=2 \sinh \left(\frac{q_{\mathrm{e}} V_{\mathrm{CT}, \mathrm{CE}}}{2 k_{\mathrm{B}} T}\right)
$$

and the activation overpotential is

$$
V_{\mathrm{CT}, \mathrm{CE}}=\frac{2 k_{\mathrm{B}} T}{q_{\mathrm{e}}} \operatorname{arcsinh}\left(\frac{i_{\mathrm{CELL}}}{2 i_{\mathrm{CE}, 0}}\right)
$$

and the charge transfer resistance can be expressed as a function of the current density

$$
R_{\mathrm{CE}}=R_{\mathrm{CT}, 0}\left[\cosh \left(\operatorname{arcsinh}\left(\frac{i_{\mathrm{CELL}}}{2 i_{\mathrm{CE}, 0}}\right)\right)\right]^{-1}
$$

The assumption that $\beta=0.5$ is well supported by experiments for Pt catalyst. ${ }^{[87]}$

\subsection{Diffusion overpotential and resistance}

In analogy with the definition of the activation overpotential, the mass transport overpotential, here the diffusion overpotential $V_{\mathrm{D}}$, is defined as the change in the electrode potential due to change in the surface concentration of the electroactive species, when the activation overpotential is assumed negligible. This occurs when the exchange current density is much larger than the current density in the cell i.e. $i_{\mathrm{CEL}} / i_{\mathrm{CE}, 0} \approx 0$. Taking this limit in Equation 54 and solving for the overpotential gives

$$
V_{\mathrm{D}, \mathrm{CE}}=\frac{k_{\mathrm{B}} T}{2 q_{\mathrm{e}}} \ln \left[\left(\frac{C_{\mathrm{I}_{3}^{-}}}{C_{\mathrm{I}_{3}^{-}}^{*}}\right)^{-1}\left(\frac{C_{\mathrm{I}^{-}}}{C_{\mathrm{I}^{-}}^{*}}\right)^{3}\right]
$$

It is worth noting that Equation 67 is identical to the Nernst equation ${ }^{[89,90]}$ describing equilibrium electrode potential, with the difference that the surface concentrations are not their equilibrium values but those influenced by the mass transport. This is understandable 
since as the rate limiting step is mass transport, the charge transfer step is essentially in equilibrium.

In the case of our simple mass transport model (Equations 58a-b) Equation 60 can be expressed as

$$
V_{\mathrm{D}, \mathrm{CE}}=\frac{k_{\mathrm{B}} T}{2 q_{\mathrm{e}}} \ln \left[\left(1-\frac{i_{\mathrm{CELL}}}{i_{\mathrm{lim}, \mathrm{I}_{3}^{-}}}\right)^{-1}\left(1+\frac{i_{\mathrm{CELL}}}{i_{\mathrm{lim}, \mathrm{I}^{-}}}\right)^{3}\right]
$$

where the limiting current densities are given by Equations 57a-b. We can also identify the separate contributions from tri-iodide and iodide diffusions in Equation 68:

$$
V_{\mathrm{D}, \mathrm{CE}}=V_{\mathrm{D}, \mathrm{IB}-}+V_{\mathrm{D}, \mathrm{I}-}
$$

where

$$
\begin{aligned}
& V_{\mathrm{D}, \mathrm{I} 3--}=\frac{k_{\mathrm{B}} T}{2 q_{\mathrm{e}}} \ln \left[\left(1-\frac{i_{\mathrm{CELL}}}{i_{\text {lim, } \mathrm{I}_{3}^{-}}}\right)^{-1}\right] \\
& V_{\mathrm{D}, \mathrm{I}-}=\frac{k_{\mathrm{B}} T}{2 q_{\mathrm{e}}} \ln \left[\left(1+\frac{i_{\mathrm{CELL}}}{i_{\mathrm{lim}, \mathrm{I}^{-}}}\right)^{3}\right]
\end{aligned}
$$

In analogy with the charge transfer resistance, the differential resistance of the diffusion overpotential defines the diffusion resistance $R_{\mathrm{D}, \mathrm{CE}}$

$$
R_{\mathrm{D}, \mathrm{CE}}=\frac{d V_{\mathrm{D}, \mathrm{CE}}}{d i_{\mathrm{CELL}}}
$$

that from based on Equations 70a-c becomes

$$
R_{\mathrm{D}, \mathrm{CE}}=R_{\mathrm{D}, \mathrm{B} 3-}+R_{\mathrm{D}, \mathrm{I}-}
$$

where 


$$
\begin{aligned}
& R_{\mathrm{D}, \mathrm{I} 3-}=\frac{k_{\mathrm{B}} T}{2 q_{\mathrm{e}}}\left[\frac{1}{i_{\text {lim, } \mathrm{I}_{3}^{-}}-i_{\mathrm{CELL}}}\right] \\
& R_{\mathrm{D}, \mathrm{I}-}=\frac{k_{\mathrm{B}} T}{2 q_{\mathrm{e}}}\left[\frac{3}{i_{\text {lim }, \mathrm{I}^{-}}+i_{\mathrm{CELL}}}\right]
\end{aligned}
$$

and the limiting current densities are given by Equations 56a-b.

\subsection{Relation between the modeled diffusion resistance at the measured diffusion impedance at the counter electrode}

In the previous Section we derived an analytical expression to the diffusion resistance of a planar DSC counter electrode starting from the general current - overpotential equation. In a typical DSC, the diffusion resistance produces an impedance arc at the low frequency end of the impedance spectrum of the cell (Figure 5) and can be analyzed by equivalent circuit fitting using the impedance function of Equation A12 in the Appendix. Note however that Equation A12 gives the diffusion impedance for a single diffusing species. The total diffusion impedance at the DSC counter electrode is in principle a sum of two expression of the type in Equation A12, one corresponding to tri-iodide and the other to the iodide diffusion, cf. ref. ${ }^{[87]}$ and page 106 in ref. ${ }^{[91]}$. With typical DSC electrolytes however, both $I^{-}$and $I_{3}^{-}$have roughly equal diffusion coefficients, and hence, their characteristic frequency of diffusion (Equation A14) is very similar. For this reason, the measured impedance spectra exhibit in practice only one diffusion impedance feature that can be very well fitted with a single expression of the type in Equation $\mathrm{A} 12$, with $R_{\mathrm{D}, \mathrm{i}}=R_{\mathrm{D}}=R_{\mathrm{D}, 13-}+R_{\mathrm{D}, \mathrm{I}-}$ and $D_{\mathrm{i}}=D_{\mathrm{I}-/ / 3-}$ interpreted as the effective electrolyte diffusion coefficient, and the diffusion layer thickness being half of the electrolyte thickness, $\delta=1 / 2 \delta_{\mathrm{EL}}$.

Furthermore, Equation A12 has been derived for the mass transport impedance at the open circuit condition, i.e. when there is zero polarization at the counter electrode, which is 
consistent with using the equilibrium concentration $C_{\mathrm{i}}^{*}$ instead of the actual current dependent surface concentration at the electrode as one of its parameter (Equation A13). Nevertheless, the same impedance function is entirely valid for analyzing the diffusion impedance also at polarized conditions, as long as it fits the impedance data well, if we acknowledge that the value of $R_{\mathrm{D}}$ depends on the current density, as predicted in the present case by Equations 73bc. Note that under the assumption of pure diffusion (Fick's law), and the symmetric planar cell geometry, the current flow in the cell does not affect the characteristic frequency of the electrolyte diffusion, since both $D_{\mathrm{I} 3-/ \mathrm{I}-}$ and $d_{\mathrm{EL}}$ remain constant in Equation A14.

Finally, it is perhaps worth mentioning that the diffusion impedance component is sometimes incorrectly placed in series with the counter electrode capacitance instead of in parallel with it as in Figure 6a. The parallel connection is the correct configuration since the voltage polarization caused by the diffusion impedance is essentially the same interfacial potential difference that controls both the charge transfer process and the charging of the double layer capacitance at the electrode - electrolyte interface. Nevertheless, the incorrect placement of the $Z_{\mathrm{D}}$ does not usually cause any problems in the equivalent circuit fitting since the diffusion and charge transfer impedances are well separated to the opposite ends of the EIS spectra (Figure 5).

\subsection{Influence of the transport mechanism and electrode porosity}

The above described mass transport model is explicitly valid only for the special case of planar (non-porous) electrodes and mass transport by pure diffusion. Several theoretical models for more detailed treatment of mass transport in DSC have been described in the literature, and will be briefly mentioned in the following. A relatively general case with respect to the cell design is the monolithic cell where all of the three active cell layers, photoelectrode, counter electrolyte and electrolyte/separator are porous films with different porosities and structure. ${ }^{[92]}$ The mass transport problem in this cell configuration was studied 
by Papageorgiou et al. ${ }^{[93]}$ assuming that electrolyte transport occurs by pure diffusion, the electrode reaction rate profile at the porous photoelectrode film is fixed and follows from the photon absorption profile, and the conductivity of the porous counter electrode film is infinite. Other cases covered in the literature include pure diffusion or mixed diffusion and migration in typical DSC type cell with planar counter electrode but with spatially uniform electron generation (weak light absorption) in the photoelectrode film, ${ }^{[90]}$ and generalization of this to different redox systems. ${ }^{[94]}$ A special case, relevant to experimental analysis of diffusion through a porous film is a CE-CE-cell with electrochemically inactive layer $\left(\mathrm{TiO}_{2}\right)$ at the one electrode. ${ }^{[95]}$ Also the electrical models of Ferber et al. involve a migration - diffusion model of the electrolyte coupled to the electron concentration in the photoelectrode film via Poisson equation within a macrohomogenous framework ${ }^{[15]}$ or via charge transfer at the $\mathrm{TiO}_{2} /$ dye/electrolyte interface. ${ }^{[17]}$

From these studies it becomes clear that the effect of porous electrodes on the mass transport in DSC is significant ${ }^{[90,93,95]}$ whereas the migration in the electrolyte can be usually neglected. ${ }^{[90]}$ The influence of the porous electrode is two-fold: firstly it offers a large active surface area for the electrode reactions, so that the reaction current density becomes distributed over the whole electrode thickness. This decreases the local current density at the reaction sites suppressing thus mass transport limitations to the electrode reactions.

Remember that the mass-transport limitations arise when the local surface concentration of one of the electroactive species reduces close to zero. For this to occur at a porous electrode, higher cell current density is required than with a planar electrode. Secondly, with a fixed substrate separation, replacing a planar electrode with a porous one decreases the effective electrode separation, and thus increases the limiting current density (Equations 57a and 57b). With the present planar model this effect can be roughly accounted for by reducing the electrolyte thickness in the model somewhat smaller than the actual substrate separation in the experimental solar cells. For the same reason, the tri-iodide diffusion limited current density 
calculated by Equation 57a gives a lower limit of the actual limiting current, when $\delta_{\mathrm{EL}}$ is the substrate separation, whereas an upper limit is obtained when $\delta_{\mathrm{EL}}$ is taken as the separation between the planar counter electrode and the edge of the porous photoelectrode.

Finally, mass transport overpotential can in fact be defined also at the photoelectrode, since also there, the concentrations of iodide and tri-iodide change due to current flow in the cell. This has an effect of shifting down the redox potential of the electrolyte at the pores of the photoelectrode film, increasing the electron recombination rate due to increased tri-iodide concentration and decreasing the regeneration rate of the oxidized dye due to decrease of iodide concentration at the photoelectode. ${ }^{[93]}$ Normally these effects are relatively small and hence neglected in the present model.

\subsection{Counter electrode IV characteristics}

Figure 14 shows the IV characteristics of the DSC counter electrode (Equation 59) and the separate contributions to it from the activation (Equation 60) and mass transport overpotentials (Equations 70a and 70b) in a $25 \mu \mathrm{m}$ thick cell for the present experimental case and two additional hypothetical cases. Firstly, it can be appreciated from Figure 14 that the division to separate activation and mass transport overpotentials is a fairly good approximation of the exact counter electrode IV curve in most cases. In Figure 14a we see that in the present case the voltage loss at the counter electrode due to mass transport in the electrolyte is almost negligible, whereas the catalytic activity $\left(R_{\mathrm{CE}, 0}\right)$ is not optimal leading to ca. $70 \mathrm{mV}$ loss at $10 \mathrm{~mA} \mathrm{~cm}$. With optimized Platinum catalyst and low viscosity acetonitrile electrolyte, or high surface area carbon counter electrodes, $R_{\mathrm{CE}, 0}$ as low as ca. 1 $\Omega \mathrm{cm}^{2}$ can be reached. ${ }^{[86,96-98]}$ This may be considered as a target value as it brings down the voltage losses close to negligible (Figure 14b). The mass transport overpotential becomes significant at current densities approaching the limiting current density. A moderate decrease of electrolyte diffusion coefficient to $1.5 \cdot 10^{-6} \mathrm{~cm}^{2} \mathrm{~s}^{-1}$ is all that is needed to reduce the 
limiting current density of the tri-iodide diffusion to $11.6 \mathrm{~mA} \mathrm{~cm}^{-2}$ (Figure $14 \mathrm{c}$ ), which would be low enough to limit $i_{\mathrm{SC}}$ of a reasonably high efficiency DSC.

Note that at sufficiently low current densities, i.e. $\left|i_{\text {CELL }}\right|<0.5\left|i_{\text {lim,I3- }}\right|$, the IV curve of the counter electrode is close to symmetric with respect to the origin (Figure 14a-b). In other words, roughly equal mass transport overpotential arises due to iodide diffusion at negative current densities as due to tri-iodide diffusion with positive currents, even though the limiting current of iodide diffusion is three times higher than that of the tri-iodide. We can understand this by Equations 73a-b according to which in a typical DSC both ions have equal contribution to $R_{\mathrm{D}}$ near zero polarization $(i=0)$, as pointed out already by Bay et al., ${ }^{[87]}$ and hence, the slopes of the respective mass transport overpotential curves are equal at $i=0$. This means that the usual assumption ${ }^{[24,27,86]}$ that the effect from the diffusion of iodide to $R_{\mathrm{D}}$ can be neglected due to their (nine times) higher equilibrium concentration compared to tri-iodide, is questionable - they have roughly an equal effect in a typical DSC. Nevertheless, as the solar cell is biased from the open circuit towards the maximum power point and the short circuit $\left(i_{\mathrm{CELL}}>>0\right)$, the mass transport resistance and overpotential at the counter electrode due to tri-iodide diffusion increases whereas that of the iodide decreases. Hence, the tri-iodide diffusion defines the limiting current density of the solar cell and dominates the mass transport overpotential at the maximum power point.

\section{Ohmic series resistance}

The Ohmic series resistance $R_{\mathrm{S}}$ of the solar cell arises mainly from the resistance of the electrolyte and the sheet resistance of the transparent conductive oxide (TCO) coated substrates: $R_{\mathrm{S}}=R_{\mathrm{EL}}+R_{\mathrm{TCO}}$. In this Section, a simple physical model is introduced for both of these resistance contributions. Contributions from the resistance of current collector lines and external wirings are neglected. 


\subsection{Electrolyte resistance}

In addition to being coupled to the charge transfer at the electrodes via mass transport, there is also an Ohmic voltage drop in the electrolyte when current passes in the cell. Neglecting again the porous photoelectrode film, the electrolyte resistance (units $\Omega \mathrm{cm}^{2}$ ) is

$$
R_{\mathrm{EL}}=\frac{d_{\mathrm{EL}}}{\kappa_{\mathrm{EL}}}=d_{\mathrm{EL}} \rho_{\mathrm{EL}}
$$

where $\kappa_{\mathrm{EL}}$ and $\rho_{\mathrm{EL}}$ are respectively the electrolyte conductivity and resistivity. The electrolyte conductivity is a function of the concentrations, diffusion coefficient and the charge of all ions $\mathrm{I}_{3}{ }^{-}, \mathrm{I}^{-}$and (unspecified) cations $\mathrm{K}^{+}\left(z_{\mathrm{I} 3-}=z_{\mathrm{I}-}=-\mathrm{zK}_{\mathrm{K}}=-1\right)$ in the electrolyte ${ }^{[88]}$

$$
\kappa_{\mathrm{EL}}=\frac{F^{2}}{R T} \sum_{\mathrm{i}}\left|z_{\mathrm{i}}\right|^{2} D_{\mathrm{i}} C_{\mathrm{i}}
$$

In the case of a the typical iodide based DSC electrolyte, as in our experimental case (Section 7), $R_{\mathrm{EL}}=0.13 \Omega \mathrm{cm}^{2}$ assuming equal diffusion coefficient for all ionic species. Hence, the Ohmic voltage loss due to a $25 \mu \mathrm{m}$ thick electrolyte layer is a negligible $1.3 \mathrm{mV}$ at $10 \mathrm{~mA} \mathrm{~cm}{ }^{-2}$ current density.

\subsection{Substrate resistance}

The photocurrent generated by DSC is collected to the external circuit via the TCO coated substrate. The sheet resistance of the TCO makes a major part of the Ohmic series resistance of the cell. The total substrate resistance $R_{\mathrm{TCO}}$ depends on the cell geometry and needs to be minimized to obtain high fill factors. Optimization of current collection becomes increasingly more important with large cells and modules ${ }^{[99]}$ but it can also significantly affect the conversion efficiency of small single cells.

Figure 15 shows typical current collection geometry in a small DSC with a stripe-like active photoelectrode film of length $L_{\text {act }}$ with collecting lines running in parallel with the film at the 
both sides of the active area of the cell. The $R_{\mathrm{TCO}}$ depends essentially on the width of the photoelectrode film $W_{\text {act }}$ and its distance $W_{\text {pass }}$ from the current collectors. Assuming that the both substrates have equal sheet resistance, and that the current collector has infinite conductivity, the current density $i_{\text {CELL }}$ is uniform over the active area of the cell, and the local current density in the TCO layer is

$$
I(y)=i_{\mathrm{CELL}} L_{\text {act }} y
$$

and runs perpendicularly to the current collector (y-direction). This introduces a voltage loss in the TCO layer beneath the active area of the cell

$$
V_{\text {act }}=\int_{0}^{W_{\text {act }}} I(y) \frac{\rho_{\mathrm{TCO}}}{d_{\mathrm{TCO}} L_{\text {act }}} d y=\int_{0}^{W_{\text {act }}} I(y) \frac{R_{\text {sheet }}}{L_{\text {act }}} d y=R_{\mathrm{TCO}, \text { act }} i_{\text {CELL }}
$$

where $R_{\text {sheet }}=\rho_{\mathrm{TCO}} / d_{\mathrm{TCO}}$ is the sheet resistance of the TCO layer having resistivity $\rho_{\mathrm{TCO}}$ and thickness $d_{\mathrm{TCO}}$, and $R_{\mathrm{TCO}}$,act the effective series resistance due to the TCO layer in the active area of the cell $\left(\Omega \mathrm{m}^{2}\right)$ given by

$$
R_{\mathrm{TCO}, \text { act }}=\frac{\Delta V_{\text {act }}}{i_{\mathrm{CELL}}}=\frac{1}{2} R_{\text {sheet }} W_{\mathrm{act}}^{2}
$$

Note that, by the nature of the calculation, this includes already both substrates.

An additional contribution to the total Ohmic voltage loss in the TCO layers comes from the current conduction over the distance ( $\left.W_{\text {pass }}\right)$ between the active area and the current collectors at the both sides of the cell

$$
V_{\text {pass }}=\left(i_{\text {CELL }} W_{\text {act }} L_{\text {act }}\right) \cdot\left(\frac{\rho_{\mathrm{TCO}}}{d_{\text {TCO }} L_{\text {act }}} 2 W_{\text {pass }}\right)=R_{\mathrm{TCO} \text {, pass }} i_{\text {CELL }}
$$

corresponding to an effective series resistance $\left(\Omega \mathrm{m}^{2}\right)$

$$
R_{\mathrm{TCO}, \text { pass }}=2 R_{\text {sheet }} W_{\text {act }} W_{\text {pass }}
$$


The total substrate resistance is the sum of the active and passive area resistances $R_{\mathrm{TCO}}=$

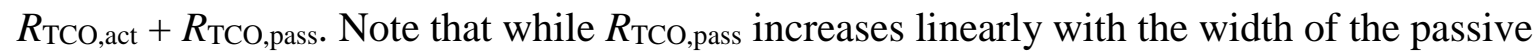
area (Equation 80$)$, the active area resistance is proportional to $\left(W_{\text {act }}\right)^{2}$, and hence, has more pronounced effect on the total $R_{\mathrm{TCO}}$ when the cell size is increased (Equation 78).

In our experimental case summarized in the next section, the TCO coated glass substrate has $R_{\text {sh }}=15 \Omega$, and $W_{\text {act }}$ and $W_{\text {pass }}$ are $5 \mathrm{~mm}$ and $4 \mathrm{~mm}$ respectively. According to the above model this gives $R_{\mathrm{TCO}, \text { act }}=1.9 \Omega \mathrm{cm}^{2}$ and $R_{\mathrm{TCO} \text {,pass }}=6.0 \Omega \mathrm{cm}^{2}$, i.e. $R_{\mathrm{TCO}}=7.9 \Omega \mathrm{cm}^{2}$ in total. Note however that this estimate is precisely valid only for a long stripe-like DSC electrode (Figure 15), e.g. a single cell in a large DSC module. In a typical small area laboratory test DSC (Figure 16) current is not confined perpendicular to the electrode edge, as in the model, but is distributed in the TCO layer, which decreases the effective TCO resistance. In the present case we find by EIS that $R_{\mathrm{S}}=4.1 \Omega \mathrm{cm}^{2}$, i.e. about half compared to the model prediction. This corresponds to a voltage loss of $41 \mathrm{mV}$ at $10 \mathrm{~mA} \mathrm{~cm}^{-2}$ current density. In addition to using a lower sheet resistance substrate, this value could be somewhat lowered by using smaller cell dimensions. However, with very small cell dimensions, the errors in the photovoltaic measurements due to diffuse light and light trapping increase and the need for proper masking of the cell is emphasized. ${ }^{[5]}$

\section{Summary of the model and comparison to experimental IV data}

In the previous Sections we used basic semiconductor physics and electrochemistry to derive a physical device model of DSC based on equivalent circuit modeling, and discussed how its parameters can be estimated experimentally using impedance spectroscopy and optical and IPCE measurements. The model is summarized in Figure $\mathbf{1 7}$ and its parameters are listed in Table 1, showing their values and the method of estimation in the present case. 
Figure 18 presents a comparison of the model to an IV curve measured from a typical small area laboratory type DSC built on glass substrates (Figure 16). Note that in this example the values of the parameters $i_{\mathrm{REC}, 0}$ and $\eta_{\mathrm{INJ}}$ are set to match the model with the measured $V_{\mathrm{OC}}$ and $i_{\mathrm{SC}}$, and hence Figure 18 serves only as a comparison of fill factor losses. The model predicts the shape of the IV curve relatively well considering that the photoelectrode parameters $L$ and $m$ were determined by EIS measurements in the dark, and the counter electrode and

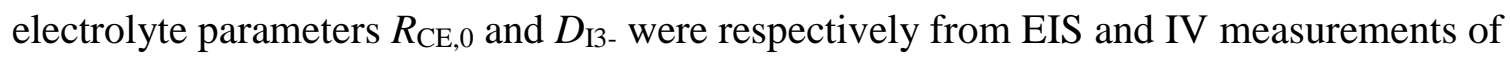
separate symmetric cells using the methods of ref. ${ }^{[86]}$. This indicates that data from separate experiments and conditions can be combined to yield reasonable predictions of the solar cell IV curve.

The simple diode model (Equation 9) produces a slightly better match to the measured IV curve than the physical equivalent circuit model (Figure 4). This is understandable since in the case of the diode model a constant series resistance was used as a fitting parameter whereas in the case of the physical model the resistance parameters were determined by independent measurements. In other words, instead of performing empirical fitting of the diode model to the IV data, the application of the present model involves determining underlying physical parameters with EIS, IPCE and optical measurements, and using this data to predict the measured IV curve, or some of its features, such as the fill factor in this case. In the present experimental case the diffusion and charge transfer resistances at the counter electrode are small enough to produce voltage losses that are roughly linear with current density (Figure 18), which explains the good fitting performance of the diode model. However, unlike the diode model, the present physical model allows factoring the IV curve (Figure 18) into its partial contributions from the different cell components, while giving them a physical meaning. 


\section{Remarks on the testing, verification and further improvement of the model}

All of the model parameters listed in Table 1 can in principle be determined by independent experiments. The model is thus fully verifiable by comparison to measured solar cell IV curves in terms of its characteristic parameters: $V_{\mathrm{OC}}, i_{\mathrm{SC}}$, and the fill factor. Perhaps the most difficult parameters to determine are $P$ and $\varepsilon$ that are needed to estimate the effect of light absorption by the electrolyte in the pores of the photoelectrode film. However, this effect is relatively small and affects only the short wavelength region. ${ }^{[38]} \mathrm{We}$ anticipate that the main challenge in the model verification will be correct independent estimation of the photocurrent $i_{\mathrm{SC}, \mathrm{TiO} 2}$ (Equations 20 and 22) applicable for the solar simulator measurements. This requires considering a number of potential error sources related to the optical characterization and $\eta_{\mathrm{LH}}$ modeling, estimation of relevant values for the $\eta_{\text {INJ }}$ and $L$ by the IPCE-ratio or other methods, ${ }^{[38,51,56,66,67]}$ and the accuracy of the solar simulator measurements that are affected by spectral mismatch error, ${ }^{[100]}$ light trapping and masking effects ${ }^{[5]}$, and the cell temperature. ${ }^{[101]}$

Since our IV model is directly related to the impedance model of DSC, we can expect it to reproduce the experimental IV curves exactly when the EIS characterization is carried out at the same conditions as the IV measurements. This has been shown in practice by others ${ }^{[28,31]}$ and is evident from Equation 14. From the point of view of model verification, the question is therefore not whether a good fit to a single IV curve can be obtained, but whether the model can explain with the same set of parameter values multiple IV curves measured at different light intensities and temperatures, and the effects of changing experimentally one model parameter while keeping the others constant. Considering the direct relation of the model to EIS, a critical test of the model will be whether it can correctly predict the current dependence of the differential resistances $R_{\mathrm{TiO} 2}, R_{\mathrm{D}, \mathrm{CE}}$ and $R_{\mathrm{CT}, \mathrm{CE}}$ measured by EIS of complete solar cells, at least to an accuracy that is sufficient for reliable IV predictions in practical cases. Detailed 
testing and verification of the model along these lines is a subject of our ongoing research, and will be carried out through application of the model to investigations of the performance and stability of new DSC materials and device structures.

Compared to other DSC models, the present model combines the best features of empirical IV models when it comes to ease of use, with more complex numerical models when it comes to quantitative physical insight. The result is inevitably a compromise between these two extremes and an approximation of the exact physical operation of the cell. In addition to the experimental testing, computational comparison with more detailed numerical DSC simulation models should be carried out to investigate where adding more complexity and physical details is necessary and justified by demostrating significantly better accuracy. Most relevant questions with this regard are the effect of electrode porosity on the mass transport in the electrolyte, discussed in Section 5.5, and the correct treatment of the non-ideality of the photoelectrode characteristics, discussed in Section 4.2, and in the recent papers of Bisquert and Mora-Seró ${ }^{[54]}$ and Villanueva-Cab et al. ${ }^{[68]}$. In both cases, investigation of the question may require numerical modeling. However, the end result should preferably be an analytical approximation that reproduces the numerical results with reasonable accuracy, so that it can be coupled with the present model to facilitate practical use.

Finally, it should be mentioned that many known factors that determine the open circuit voltage of DSCs are integrated in the present model in just three parameters: $i_{\mathrm{REC}, 0}$ and $m$ that describe the electron recombination and $L$ that describes their distribution in the photoelectrode film by diffusion. For example, effects of the $\mathrm{TiO}_{2}$ conduction band edge potential, the redox potential of the electrolyte, and the electron recombination rate constant are all embedded in the value of $i_{\mathrm{REC}, 0}$ (Equations 27a and 37). Also the electron injection efficiency that is treated here as a single parameter, is known to be sensitive not only to the properties of the dye but also to the mutual energetic and structural conditions at the $\mathrm{TiO}_{2}-$ dye - electrolyte interface. ${ }^{[52]}$ The contributions from these and other effects on the $V_{\mathrm{OC}}$ and 
$\eta_{\mathrm{INJ}}$ of DSCs should be factored out by a common physical model and integrated into the present model.

\section{Conclusions}

When light hits DSC, it produces an open circuit voltage as a result of a balance between electron generation and recombination at the photoelectrode. When current is drawn from the cell under load conditions, resistances of the different cell components cause voltage losses that decrease the cell voltage and determine the IV curve and the power output of the solar cell. In this paper, the basic physical and electrochemical principles of DSC were reviewed and applied to derive a complete physical IV and resistance model of DSC using equivalent circuit impedance modeling as a starting point. For this purpose mathematical expressions for the IV curve and for its differential resistance corresponding to all resistive equivalent circuit components were derived and their relation to the conventional impedance modeling was explained. A number of assumption and simplifications were introduced in the model to reach analytical solutions, and their effect on the accuracy of the model was discussed qualitatively. Future work should investigate these effects quantitatively to evaluate the need for more accurate numerical modeling of the DSC.

The presented model has several features that render it particularly useful to scientists developing and testing advanced materials for DSCs. The model can be verified and its key parameters estimated by widely available experimental techniques: electrochemical impedance spectroscopy (EIS), optical characterization, and spectral incident-photon-tocollected-electron (IPCE) measurements. Data from these measurements can be combined to create predictions of the cell efficiency and identify best strategies to improve it. Since the model is based on analytical equations it can be implemented easily as spreadsheet calculations. Most importantly, the model links the physical parameters of DSC with impedance parameters that can be determined by non-destructive current dependent EIS 
measurements of complete solar cells. This opens up the possibility to monitor physical solar cell parameters in the production lines and during long term stability testing of DSCs.

\section{Acknowledgements}

One of the authors (J.H.) thanks Prof. Juan Bisquert from Univ. of Jaume and Prof. Laurie Peter from Univ. Bath for fruitful discussions on the background and importance of the nonideality of DSCs. The work was partially funded by the Multidisciplinary Institute of Digitalization and Energy (MIDE) of the Helsinki University of Technology. J.H. and K.M. thank the Graduate School of Energy Technology (EST) for their scholarships.

Received: ((will be filled in by the editorial staff))

Revised: ((will be filled in by the editorial staff))

Published online: ((will be filled in by the editorial staff)) 


\section{APPENDIX I}

\section{Transmission line impedance of the photoelectrode}

According to the transmission line model, the impedance of the DSC photoelectrode is ${ }^{[25]}$

$$
Z_{\mathrm{TiO} 2}=\sqrt{\frac{R_{\mathrm{T}} R_{\mathrm{REC}}}{1+\left(i \omega / \omega_{\mathrm{REC}}\right)^{\beta_{\mathrm{CPE}}}}} \operatorname{coth}\left(\left(\omega_{\mathrm{REC}} / \omega_{\mathrm{L}}\right)^{\beta_{\mathrm{CPE}} / 2} \sqrt{1+\left(i \omega / \omega_{\mathrm{REC}}\right)^{\beta_{\mathrm{CPE}}}}\right)
$$

where

$$
\omega_{\mathrm{REC}}=\frac{1}{\left(R_{\mathrm{REC}} Q_{\mathrm{CPE}}\right)^{1 / \beta_{\mathrm{CPE}}}}=\frac{1}{\left(r_{\mathrm{REC}} q_{\mathrm{CPE}}\right)^{1 / \beta_{\mathrm{CPE}}}}
$$

is the characteristic frequency of the charge transfer (recombination) process, and

$$
\omega_{\mathrm{T}}=\frac{1}{\left(R_{\mathrm{T}} Q_{\mathrm{CPE}}\right)^{1 / \beta_{\mathrm{CPE}}}}=\frac{1}{\left(r_{\mathrm{T}} q_{\mathrm{CPE}} d^{2}\right)^{1 / \beta_{\mathrm{CPE}}}}
$$

is the characteristic frequency of the electron transport process. The parameters $Q_{\mathrm{CPE}} \beta_{\mathrm{CPE}}$ are the pre-factor and exponent of the constant phase impedance element

$$
Z_{\mathrm{CPE}}=\frac{1}{Q_{\mathrm{CPE}}}(\mathrm{i} \omega)^{-\beta_{\mathrm{CPE}}}
$$

that is required to describe the frequency dispersion omnipresent in experimental impedance data. This dispersion is characterized by the exponent $\beta_{\mathrm{CPE}}$ that typically takes values in the range $0.85<\beta_{\mathrm{CPE}}<0.95$. The (chemical) capacitance corresponding to the CPE element may be estimated as

$$
C_{\mu}=Q_{\mathrm{CPE}}^{1 / \beta_{\mathrm{CPE}}} R_{\mathrm{REC}}^{1 / \beta_{\mathrm{CPE}}^{-1}}
$$

by requiring that the capacitance reproduces equal time constant than the CPE element ${ }^{[25]}$ when connected in parallel with the electron recombination resistance:

$$
\tau_{\mathrm{R}-\mathrm{CPE}}=\left(R_{\mathrm{REC}} Q_{\mathrm{CPE}}\right)^{1 / \beta_{\mathrm{CPE}}} \equiv \tau_{\mathrm{R}-\mathrm{C}}=R_{\mathrm{REC}} C_{\mu}
$$


Note that $\omega_{\mathrm{REC}}$ corresponds to a relaxation time constant $\tau_{\mathrm{REC}}=\omega_{\mathrm{REC}}{ }^{-1}$ that is often interpreted as the electron lifetime in the photoelectrode. ${ }^{[72]}$ The details of the frequency dependence of Equation A1 are described by Bisquert et al.. ${ }^{[25,69]}$

In this paper, our main interest is in the steady state (DC) resistance of the photoelectrode film and its measurement by impedance spectroscopy. According to transmission line model this is given by $(\omega \rightarrow 0$ in Equation A1)

$$
R_{\mathrm{TiO} 2}=\sqrt{R_{\mathrm{T}} R_{\mathrm{REC}}} \operatorname{coth}\left(\sqrt{\frac{R_{\mathrm{T}}}{R_{\mathrm{REC}}}}\right)
$$

The photoelectrode resistance is thus determined by the electron transport and recombination resistances that can be extracted from experimental impedance data whenever the data exhibits their characteristic features: $R_{\mathrm{T}}$ produces a linear part in the complex plane impedance plot, with an angle of ca. $45^{\circ}$ (depending on the value of $\beta_{\mathrm{CPE}}$ ), ${ }^{[25]}$ whereas $R_{\mathrm{REC}}$ produces an impedance arc. The length along the real axis of the linear transport part is $1 / 3 R_{\mathrm{T}}$ and the width of the recombination arc is $R_{\text {REC }}$. Hence, the total photoelectrode resistance can be approximated as

$$
R_{\mathrm{TiO} 2}=\frac{1}{3} R_{\mathrm{T}}+R_{\mathrm{REC}}=\frac{1}{3} r_{\mathrm{T}} d+\frac{r_{\mathrm{REC}}}{d} \quad\left(R_{\mathrm{REC}}>R_{\mathrm{T}}\right)
$$

Note that while the recombination arc is always visible in the EIS data of a DSC, the $R_{\mathrm{T}}$ may not be visible for two reasons. Firstly, it can be negligibly small and thus overlap with counter electrode impedance arc (as in Figure 5). Secondly, it may be larger than the recombination resistance, which is a fingerprint of a poor quality photoelectrode. In this case, Equation A1 reduces to a form similar to a Gerischer impedance

$$
Z_{\mathrm{TiO} 2}=\frac{R_{\mathrm{TiO} 2}}{\sqrt{1+\left(\mathrm{i} \omega / \omega_{\mathrm{REC}}\right)^{\beta_{\mathrm{CPE}}}}} \quad\left(R_{\mathrm{REC}}<R_{\mathrm{T}}\right)
$$


Equation A9 represents in the complex plane plot a skewed impedance arc with width $R_{\mathrm{TiO} 2}$ and a linear part at high frequencies. ${ }^{[25]}$ Note that Equation A9 has only one independent resistance parameter, the total photoelectrode resistance

$$
R_{\mathrm{TiO} 2}=\sqrt{R_{\mathrm{T}} R_{\mathrm{REC}}}=\sqrt{r_{\mathrm{T}} r_{\mathrm{REC}}} \quad\left(R_{\mathrm{REC}}<R_{\mathrm{T}}\right) .
$$

In other words, whenever the impedance spectrum is well described by Equation A9, neither $R_{\mathrm{T}}$ nor $R_{\mathrm{REC}}$ can be determined from the data - but only their product. Fitting Equation A1 to the impedance spectrum would still be technically possibly, but there would be redundancy between the fitting parameters $R_{\mathrm{T}}$ and $R_{\mathrm{REC}}$ and thus large errors in their values. The reason to the failure of EIS to distinguish between $R_{\mathrm{T}}$ and $R_{\mathrm{REC}}$ when $R_{\mathrm{T}}>R_{\mathrm{REC}}$ is that the electron diffusion length $L^{[25,69]}$

$$
L=d \sqrt{\frac{R_{\mathrm{REC}}}{R_{\mathrm{T}}}}=\sqrt{\frac{r_{\mathrm{REC}}}{r_{\mathrm{T}}}}
$$

is shorter than the film thickness $d$. This means that the current injected into the film from the contact does not reach to the other end of the film, but is transferred to the electrolyte roughly within the distance $L$ from the contact. In accordance with this, Equation A10 is not sensitive to the film thickness, contrary to Equation A8. This means that when $L<d$, the resistance of the whole film is not measurable by EIS, but only a fraction of it, this fraction remaining unknown. Note that the same applies also to the capacitance of the film. Interestingly, the electron lifetime $\tau=\omega_{\mathrm{REC}^{-1}}$ is nevertheless still measurable (Equation A9).

\section{Diffusion impedance at the counter electrode}

Assuming Fick's law of diffusion in a symmetric thin layer electrochemical cell with planar electrodes, the mass transport impedance due to diffusion of one electroactive species (i) in the electrolyte can be described by the so called finite length Warburg impedance with absorbing (short circuited) boundary condition, cf. $^{[27,86,91]}$ 


$$
Z_{\mathrm{D}, \mathrm{i}}=R_{\mathrm{D}, \mathrm{i}} \sqrt{\frac{\omega_{\mathrm{D}, \mathrm{i}}}{i \omega}} \tanh \left(\sqrt{\frac{i \omega}{\omega_{\mathrm{D}, \mathrm{i}}}}\right)
$$

where $R_{\mathrm{D}, \mathrm{i}}$ is the diffusion resistance of species (i) given by

$$
R_{\mathrm{D}, \mathrm{i}}=\frac{k_{\mathrm{B}} T \delta}{n^{2} q_{e}^{2} C_{\mathrm{i}}^{*} D_{\mathrm{i}}}
$$

and

$$
\omega_{\mathrm{D}, \mathrm{i}}=\frac{D_{\mathrm{i}}}{\delta^{2}}
$$

is the characteristic frequency of the diffusion process. Here, $n$ is the number of electrons transferred in the overall electrode reaction, and $C_{\mathrm{i}}{ }^{*}$ the steady state bulk concentration of the species $\mathrm{i}$. The characteristic frequency $\omega_{\mathrm{D}, \mathrm{i}}$ depends on the diffusion coefficient $D_{\mathrm{i}}$ of the electroactive ion and the thickness of the diffusion layer $\delta$. The diffusion layer thickness corresponds to the distance from the electrode where the AC modulated concentration of the redox species remains unchanged compared to its equilibrium value, which in the case of the symmetric thin layer cell equals half of the electrolyte layer thickness, i.e. $\delta=1 / 2 d_{\mathrm{EL}}$ according to mass transport model in this paper.

In the complex plane impedance plot Equation A12 represents a distorted semicircle with width $R_{\mathrm{D} \text {,i. }}$ The apex of the arc, corresponding to a peak in the negative imaginary part vs frequency plot, is at frequency $f_{\mathrm{D}, \mathrm{i}}=\omega_{\mathrm{D}, \mathrm{i}}(2 \pi)^{-1} \cdot{ }^{[85]}$ 


\section{References}

[1] B. O'Regan, M. Grätzel, Nature 1991, 353, 737.

[2] K. Hara, H. Arakawa, in Handbook of Photovoltaic Science and Engineering, (Eds: A. Luque, S. Hegedus), John Wiley \& Sons, West Sussex, England 2003, Ch. 15.

[3] L. M. Peter, Phys. Chem. Chem. Phys. 2007, 9, 2630.

[4] S. Ito, T. N. Murakami, P. Comte, P. Liska, C. Grätzel, M. K. Nazeeruddin, M. Grätzel, Thin Solid Films 2008, 516, 4613.

[5] S. Ito, K. Nazeeruddin, P. Liska, P. Comte, R. Charvet, P. Pechy, M. Jirousek, A. Kay, S. M. Zakeeruddin, M. Grätzel, Progress in Photovoltaics 2006, 14, 589.

[6] Z. Wang, H. Kawauchi, T. Kashima, H. Arakawa, Coord. Chem. Rev. 2004, 248, 1381.

[7] J. M. Kroon, N. J. Bakker, H. J. P. Smit, P. Liska, K. R. Thampi, P. Wang, S. M.

Zakeeruddin, M. Grätzel, A. Hinsch, S. Hore, U. Würfel, R. Sastrawan, J. R. Durrant, E. Palomares, H. Pettersson, T. Gruszecki, J. Walter, K. Skupien, G. E. Tulloch, Progress in Photovoltaics 2007, 15, 1.

[8] D. Shi, N. Pootrakulchote, R. Li, J. Guo, Y. Wang, S. M. Zakeeruddin, M. Grätzel, P. Wang, J. Phys. Chem. C 2008, 112, 17046.

[9] J. Desilvestro, M. Bertoz, S. Tulloch, G. Tulloch, "Packaging, scaling and commercialization of Dye Solar Cells", Presentation at E-MRS 2009 Spring Meeting, Strasbourg, June 8 - 12, 2009.

[10] N. Koide, A. Islam, Y. Chiba, L. Han, J. Photochem. Photobiol., A 2006, 182, 296.

[11] H. Tian, X. Zhang, S. Yuan, X. Wang, Z. Tian, B. Liu, Y. Wang, T. Yu, Z. Zou, Sol. Energy 2009, 83, 715.

[12] M. Murayama, T. Mori, Thin Solid Films 2006, 509, 123.

[13] G. Boschloo, H. Lindström, E. Magnusson, A. Holmberg, A. Hagfeldt, J. Photochem. Photobiol., A 2002, 148, 11. 
[14] A. Hagfeldt, G. Boschloo, H. Lindström, E. Figgemeier, A. Holmberg, V. Aranyos, E. Magnusson, L. Malmqvist, Coord. Chem. Rev. 2004, 248, 1501.

[15] J. Ferber, R. Stangl, J. Luther, Sol. Energy Mater. Sol. Cells 1998, 53, 29.

[16] S. Tanaka, Jpn. J. Appl. Phys. 2001, 40, 97.

[17] J. Ferber, J. Luther, J. Phys. Chem. B 2001, 105, 4895.

[18] M. Penny, T. Farrell, G. Will, Sol. Energy Mater. Sol. Cells 2008, 92, 24.

[19] J. Villanueva, J. A. Anta, E. Guillen, G. Oskam, J. Phys. Chem. C 2009, 113, 19722.

[20] D. Matthews, P. Infelta, M. Grätzel, Sol. Energy Mater. Sol. Cells 1996, 44, 119.

[21] S. Y. Huang, G. Schlichthoerl, A. J. Nozik, M. Grätzel, A. J. Frank, J. Phys. Chem. B 1997, 101, 2576.

[22] M. Penny, T. Farrell, C. Please, Sol. Energy Mater. Sol. Cells 2008, 92, 11.

[23] J. Lee, G. M. Coia, N. S. Lewis, J. Phys. Chem. B 2004, 108, 5269.

[24] R. Kern, R. Sastrawan, J. Ferber, R. Stangl, J. Luther, Electrochim. Acta 2002, 47, 4213.

[25] J. Bisquert, G. Garcia-Belmonte, F. Fabregat-Santiago, N. S. Ferriols, P. Bogdanoff, E. C. Pereira, J. Phys. Chem. B 2000, 104, 2287.

[26] F. Fabregat-Santiago, J. Bisquert, G. Garcia-Belmonte, G. Boschloo, A. Hagfeldt, Sol. Energy Mater. Sol. Cells 2005, 87, 117.

[27] Q. Wang, J. Moser, M. Grätzel, J. Phys. Chem. B 2005, 109, 14945.

[28] F. Fabregat-Santiago, J. Bisquert, E. Palomares, L. Otero, D. Kuang, S. M. Zakeeruddin, M. Grätzel, J. Phys. Chem. C 2007, 111, 6550.

[29] K. Miettunen, J. Halme, M. Toivola, P. Lund, J. Phys. Chem. C 2008, 112, 4011.

[30] K. Miettunen, J. Halme, P. Vahermaa, T. Saukkonen, M. Toivola, P. Lund, J. Electrochem. Soc. 2009, 156, B876.

[31] Q. Wang, S. Ito, M. Grätzel, F. Fabregat-Santiago, I. Mora-Sero, J. Bisquert, T. Bessho, H. Imai, J. Phys. Chem. B 2006, 110, 25210. 
[32] T. C. Li, M. S. Goes, F. Fabregat-Santiago, J. Bisquert, P. R. Bueno, C. Prasittichai, J. T. Hupp, T. J. Marks, J. Phys. Chem. C 2009, 113, 18385.

[33] S. Södergren, A. Hagfeldt, J. Olsson, S. Lindquist, J. Phys. Chem. 1994, 98, 5552.

[34] L. Han, N. Koide, Y. Chiba, A. Islam, T. Mitate, C. R. Chim. 2006, 9, 645.

[35] L. Bay, K. West, Sol. Energy Mater. Sol. Cells 2005, 87, 613.

[36] J. Bisquert, Phys. Chem. Chem. Phys. 2003, 5, 5360.

[37] T. Hoshikawa, M. Yamada, R. Kikuchi, K. Eguchi, J. Electrochem. Soc. 2005, 152, E68.

[38] J. Halme, G. Boschloo, A. Hagfeldt, P. Lund, J. Phys. Chem. C 2008, 112, 5623.

[39] P. M. Sommeling, H. C. Rieffe, J. A. M. van Roosmalen, A. Schonecker, J. M. Kroon, J. A. Wienke, A. Hinsch, Sol. Energy Mater. Sol. Cells 2000, 62, 399.

[40] A. C. Fisher, L. M. Peter, E. A. Ponomarev, A. B. Walker, K. G. U. Wijayantha, J. Phys. Chem. B 2000, 104, 949.

[41] T. Trupke, P. Wrfel, I. Uhlendorf, J. Phys. Chem. B 2000, 104, 11484.

[42] J. Halme, K. Miettunen, P. Lund, J. Phys. Chem. C 2008, 112, 20491.

[43] G. Rothenberger, P. Comte, M. Grätzel, Sol. Energy Mater. Sol. Cells 1999, 58, 321.

[44] Y. Tachibana, K. Hara, K. Sayama, H. Arakawa, Chem. Mater. 2002, 14, 2527.

[45] K. Keis, A. Roos, Optical Materials 2002, 20, 35.

[46] R. Argazzi, C. A. Bignozzi, M. Yang, G. M. Hasselmann, G. J. Meyer, Nano Lett. 2002, 2,625 .

[47] D. F. Watson, G. J. Meyer, Annu. Rev. Phys. Chem. 2005, 56, 119.

[48] J. R. Durrant, S. A. Haque, E. Palomares, Coord. Chem. Rev. 2004, 248, 1247.

[49] N. A. Anderson, T. Lian, Annu. Rev. Phys. Chem. 2005, 56, 491.

[50] S. E. Koops, J. R. Durrant, Inorg. Chim. Acta 2008, 361, 663.

[51] P. R. F. Barnes, A. Y. Anderson, S. E. Koops, J. R. Durrant, B. C. O'Regan, J. Phys. Chem. C 2009, 113, 1126. 
[52] S. E. Koops, B. C. O'Regan, P. R. F. Barnes, J. R. Durrant, J. Am. Chem. Soc. 2009, 131, 4808 .

[53] C. Serpa, J. Schabauer, A. P. Piedade, C. J. P. Monteiro, M. M. Pereira, P. Douglas, H. D. Burrows, L. G. Arnaut, J. Am. Chem. Soc. 2008, 130, 8876.

[54] J. Bisquert, I. Mora-Sero, J. Phys. Chem. Lett. 2010, 1, 450.

[55] L. Dloczik, O. Ileperuma, I. Lauermann, L. M. Peter, E. A. Ponomarev, G. Redmond, N. J. Shaw, I. Uhlendorf, J. Phys. Chem. B 1997, 101, 10281.

[56] H. K. Dunn, L. M. Peter, J. Phys. Chem. C 2009, 113, 4726.

[57] G. Franco, J. Gehring, L. M. Peter, E. A. Ponomarev, I. Uhlendorf, J. Phys. Chem. B 1999, 103, 692.

[58] G. Schlichthörl, S. Y. Huang, J. Sprague, A. J. Frank, J. Phys. Chem. B 1997, 101, 8141.

[59] N. W. Duffy, L. M. Peter, K. G. U. Wijayantha, Electrochem. Comm. 2000, 2, 262.

[60] A. Zaban, M. Greenshtein, J. Bisquert, ChemPhysChem 2003, 4, 859.

[61] S. Nakade, W. Kubo, Y. Saito, T. Kanzaki, T. Kitamura, Y. Wada, S. Yanagida, J. Phys. Chem. B 2003, 107, 14244.

[62] S. Nakade, T. Kanzaki, Y. Wada, S. Yanagida, Langmuir 2005, 21, 10803.

[63] J. Nissfolk, K. Fredin, A. Hagfeldt, G. Boschloo, J. Phys. Chem. B 2006, 110, 17715.

[64] B. C. O'Regan, K. Bakker, J. Kroeze, H. Smit, P. Sommeling, J. R. Durrant, J. Phys. Chem. B 2006, 110, 17155.

[65] J. Bisquert, V. S. Vikhrenko, J. Phys. Chem. B 2004, 108, 2313.

[66] P. R. F. Barnes, L. Liu, X. Li, A. Y. Anderson, H. Kisserwan, T. H. Ghaddar, J. R.

Durrant, B. C. O'Regan, Nano Lett. 2009, 9, 3532.

[67] H. Wang, L. M. Peter, J. Phys. Chem. C 2009, 113, 18125.

[68] J. Villanueva-Cab, H. Wang, G. Oskam, L.M. Peter, J. Phys. Chem. Lett., 2010, 1, 748

[69] J. Bisquert, J. Phys. Chem. B 2002, 106, 325. 
[70] A. Pitarch, G. Garcia-Belmonte, I. Mora-Sero, J. Bisquert, Phys. Chem. Chem. Phys. 2004, 6, 2983.

[71] J. Bisquert, J. Phys. Chem. B 2004, 108, 2323.

[72] J. Bisquert, F. Fabregat-Santiago, I. Mora-Sero, G. Garcia-Belmonte, S. Gimenez, J. Phys. Chem. C 2009, 113, 17278.

[73] K. Lobato, L. M. Peter, U. Würfel, J. Phys. Chem. B 2006, 110, 16201.

[74] U. Würfel, J. Wagner, A. Hinsch, J. Phys. Chem. B 2005, 109, 20444.

[75] P. J. Cameron, L. M. Peter, S. Hore, J. Phys. Chem. B 2005, 109, 930.

[76] L. M. Peter, J. Phys. Chem. C 2007, 111, 6601.

[77] J. Xia, N. Masaki, K. Jiang, S. Yanagida, J. Phys. Chem. C 2007, 111, 8092.

[78] S. Hore, R. Kern, Appl. Phys. Lett. 2005, 87, 263504/1.

[79] G. Boschloo, A. Hagfeldt, Acc. Chem. Res. 2009, 42, 1819.

[80] P. Salvador, M. G. Hidalgo, A. Zaban, J. Bisquert, J. Phys. Chem. B 2005, 109, 15915.

[81] J. R. Jennings, A. Ghicov, L. M. Peter, P. Schmuki, A. B. Walker, J. Am. Chem. Soc. 2008, 130, 13364.

[82] N. W. Duffy, L. M. Peter, R. M. G. Rajapakse, K. G. U. Wijayantha, J. Phys. Chem. B 2000, 104, 8916.

[83] L. Peter, Acc. Chem. Res. 2009, 42, 1839.

[84] B. C. O'Regan, J. R. Durrant, Acc. Chem. Res. 2009, 42, 1799.

[85] J. A. Anta, F. Casanueva, G. Oskam, J. Phys. Chem. B 2006, 110, 5372.

[86] A. Hauch, A. Georg, Electrochim. Acta 2001, 46, 3457.

[87] L. Bay, K. West, B. Winther-Jensen, T. Jacobsen, Sol. Energy Mater. Sol. Cells 2006, 90, 341.

[88] A. J. Bard, L. R. Faulkner, Electrochemical methods: fundamentals and applications, 2nd Ed, Wiley, New York, 2000 
[89] N. Papageorgiou, Y. Athanassov, M. Armand, P. Bonhote, H. Pettersson, A. Azam, M. Grätzel, J. Electrochem. Soc. 1996, 143, 3099.

[90] N. Papageorgiou, M. Grätzel, P. P. Infelta, Sol. Energy Mater. Sol. Cells 1996, 44, 405.

[91] J. R. Macdonald, Ed., Impedance spectroscopy: empasizing solid materials and systems, Wiley, New York, 1987.

[92] A. Kay, M. Grätzel, Sol. Energy Mater. Sol. Cells 1996, 44, 99.

[93] N. Papageorgiou, P. Liska, A. Kay, M. Grätzel, J. Electrochem. Soc. 1999, 146, 898.

[94] W. Hyk, J. Augustynski, J. Electrochem. Soc. 2006, 153, A2326.

[95] N. Papageorgiou, C. Barbe, M. Grätzel, J. Phys. Chem. B 1998, 102, 4156.

[96] N. Papageorgiou, W. F. Maier, M. Grätzel, J. Electrochem. Soc. 1997, 144, 876.

[97] T. N. Murakami, M. Grätzel, Inorg. Chim. Acta 2008, 361, 572.

[98] J. Halme, M. Toivola, A. Tolvanen, P. Lund, Sol. Energy Mater. Sol. Cells 2006, 90, 872.

[99] M. Toivola, T. Peltola, K. Miettunen, J. Halme, P. Lund, Journal of Nanosci.

Nanotechnol. 2010, 10, 1.

[100] S. Ito, H. Matsui, K. Okada, S. Kusano, T. Kitamura, Y. Wada, S. Yanagida, Sol.

Energy Mater. Sol. Cells 2004, 82, 421.

[101] M. Toivola, L. Peltokorpi, J. Halme, P. Lund, Sol. Energy Mater. Sol. Cells 2007, 91, 1733. 


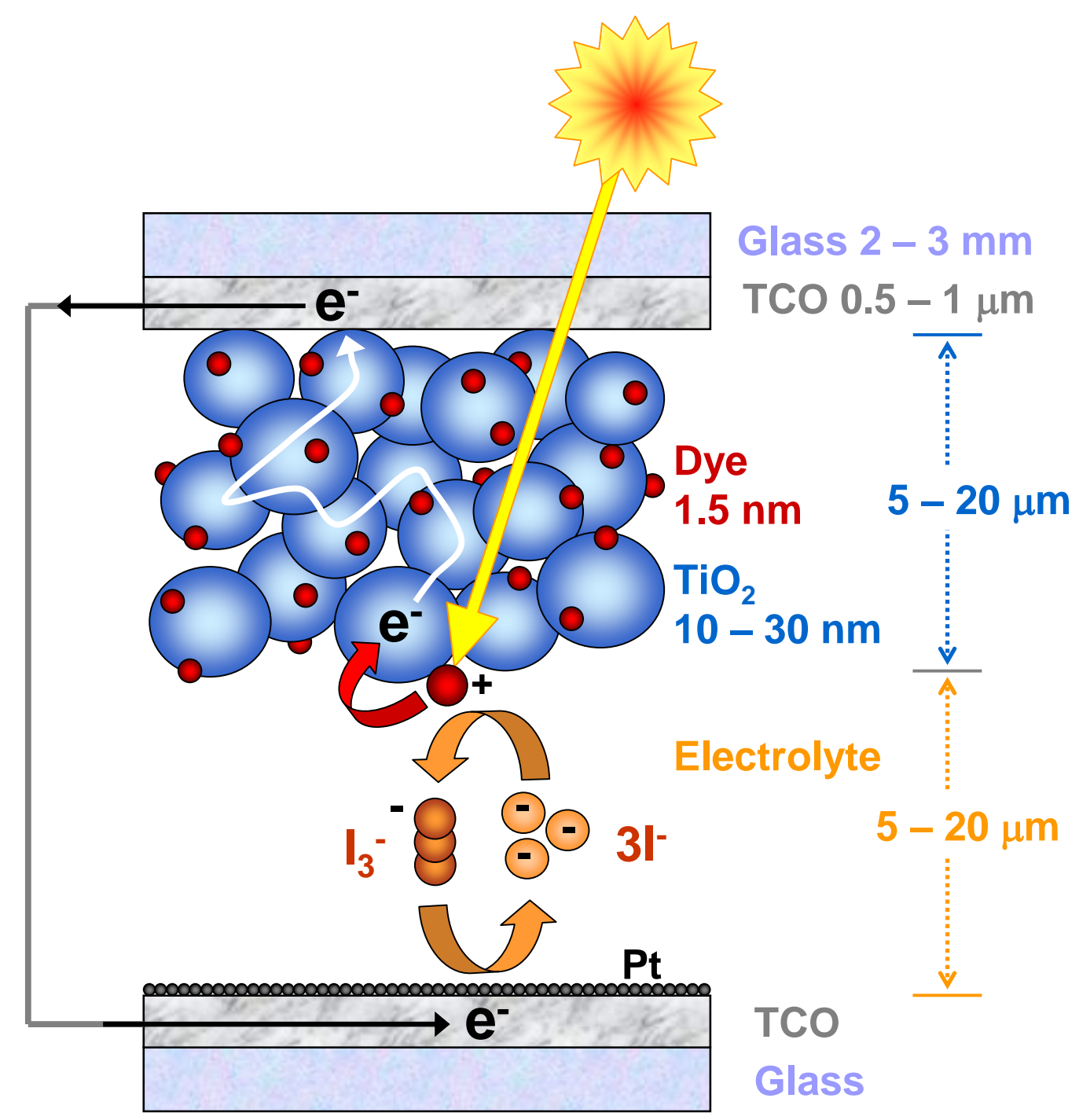

Figure 1. The structure and operation of the dye solar cell. 


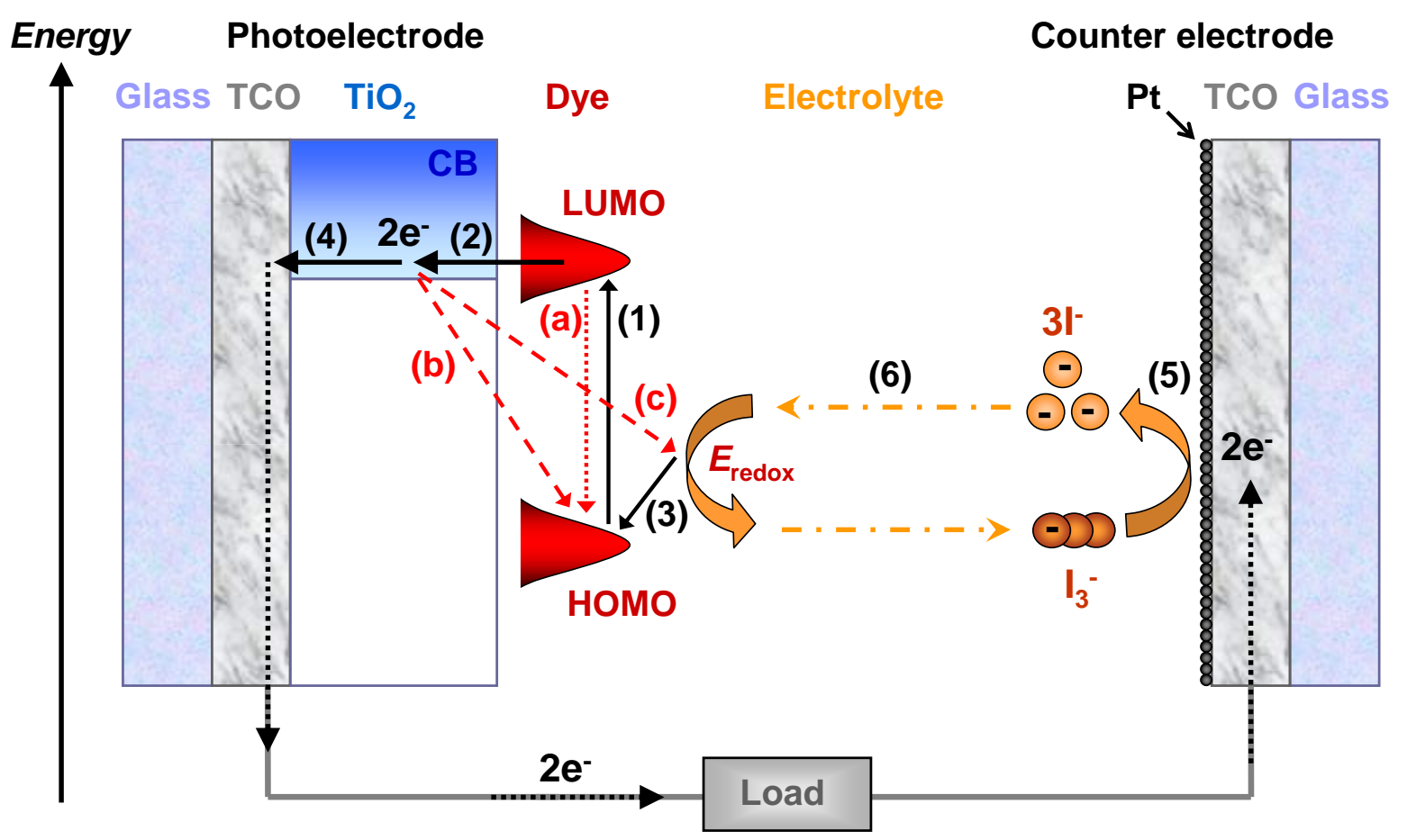

Figure 2. Operating principle of the dye solar cell, showing the forward reactions referred to by numbers in the text. The main back-reactions limiting the photocurrent are indicated with red arrows: (a) radiationless relaxation of the excited state of the dye, (b) recombination of the electrons with the oxidized dye, (c) and with the tri-iodide in the electrolyte.

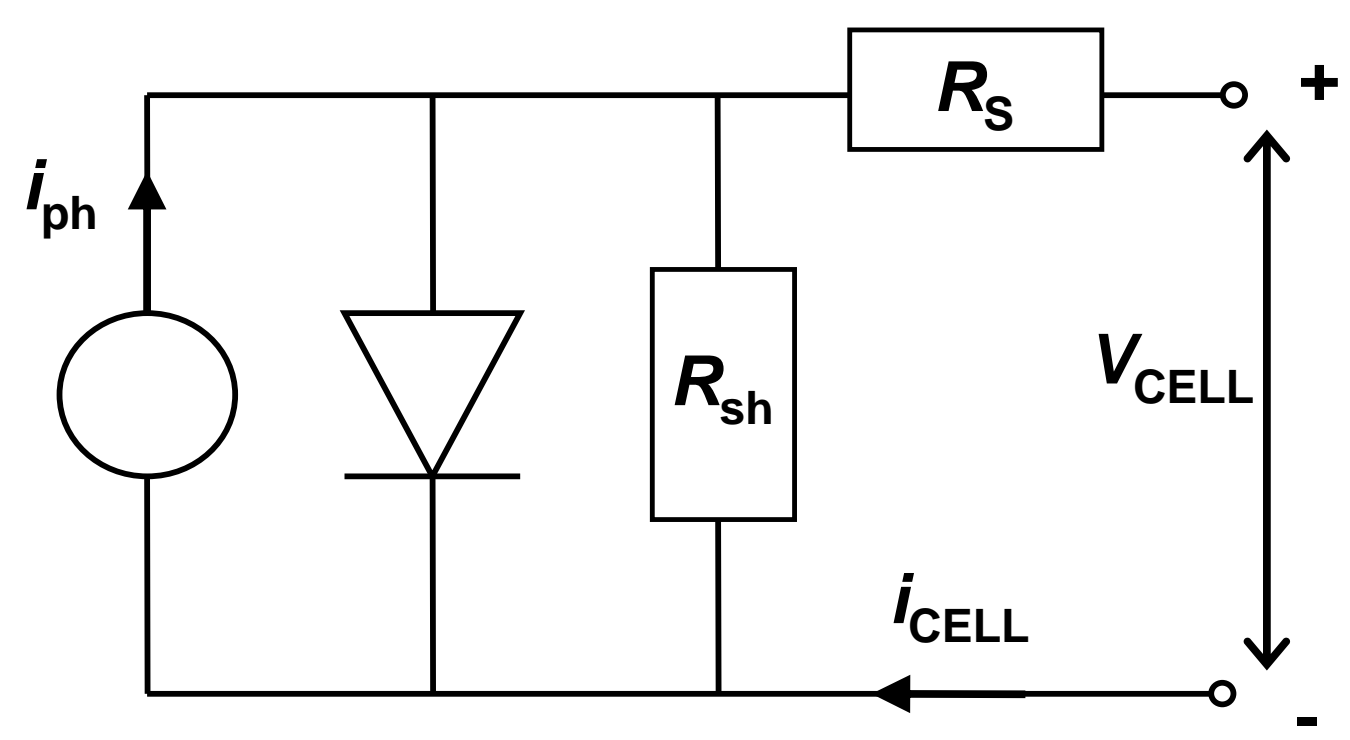

Figure 3. Basic diode equivalent circuit model of a solar cell. 


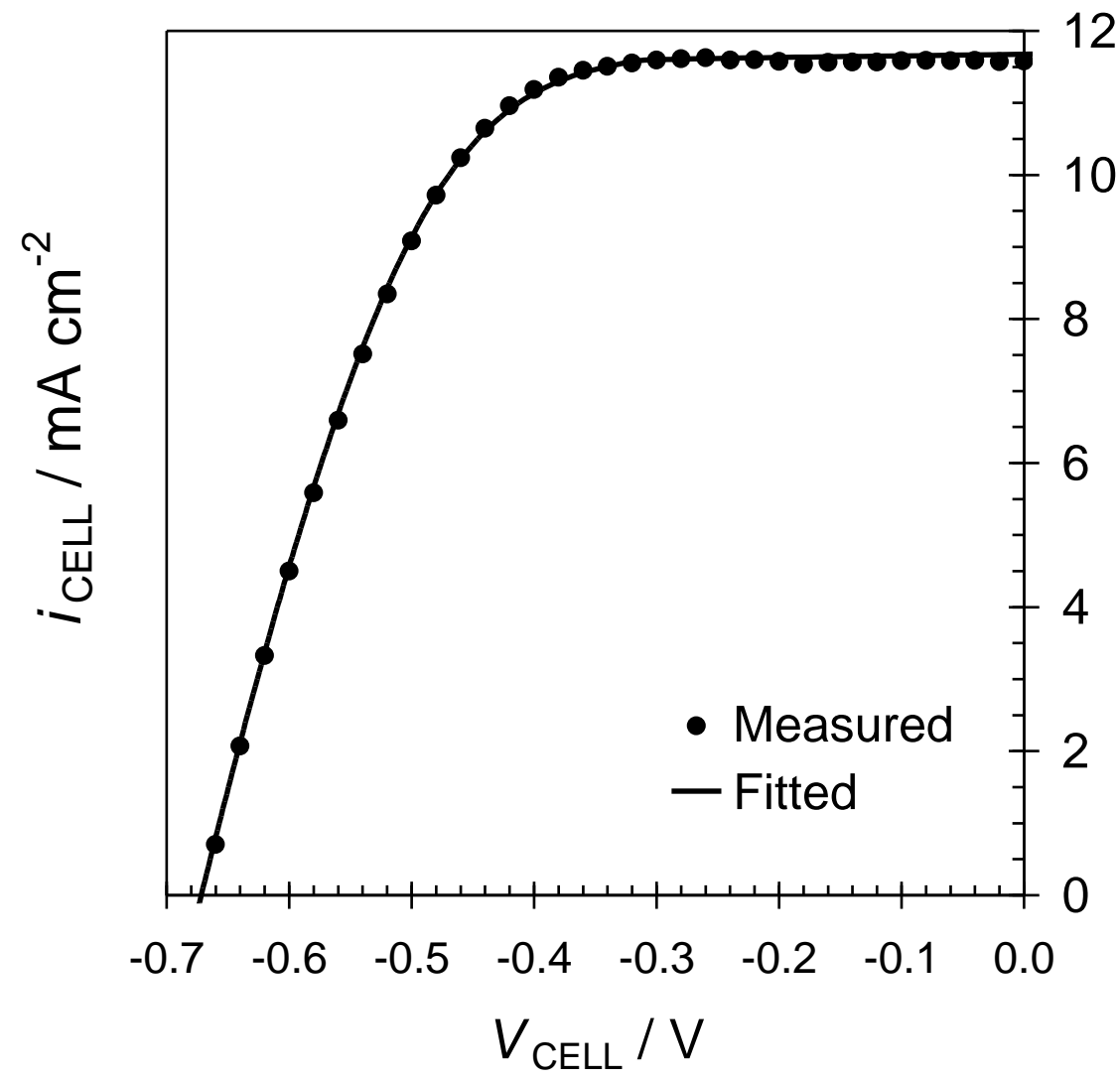

Figure 4. The diode model (Equation 9) fitted to IV curve of DSC measured at $1000 \mathrm{~W} \mathrm{~m}^{-2}$ AM1.5G (standard solar spectral irradiance) equivalent light intensity. The diode model was fitted using $m=2.1$ and $R_{\mathrm{sh}}=\infty$ as a fixed parameters, while treating the other parameters free taking values: $i_{\mathrm{ph}}=11.7 \mathrm{~mA} \mathrm{~cm}{ }^{-2}, i_{0}=3.7 \cdot 10^{-5} \mathrm{~mA} \mathrm{~cm}{ }^{-2}, R_{\mathrm{S}}=10 \Omega \mathrm{cm}^{2}$. 


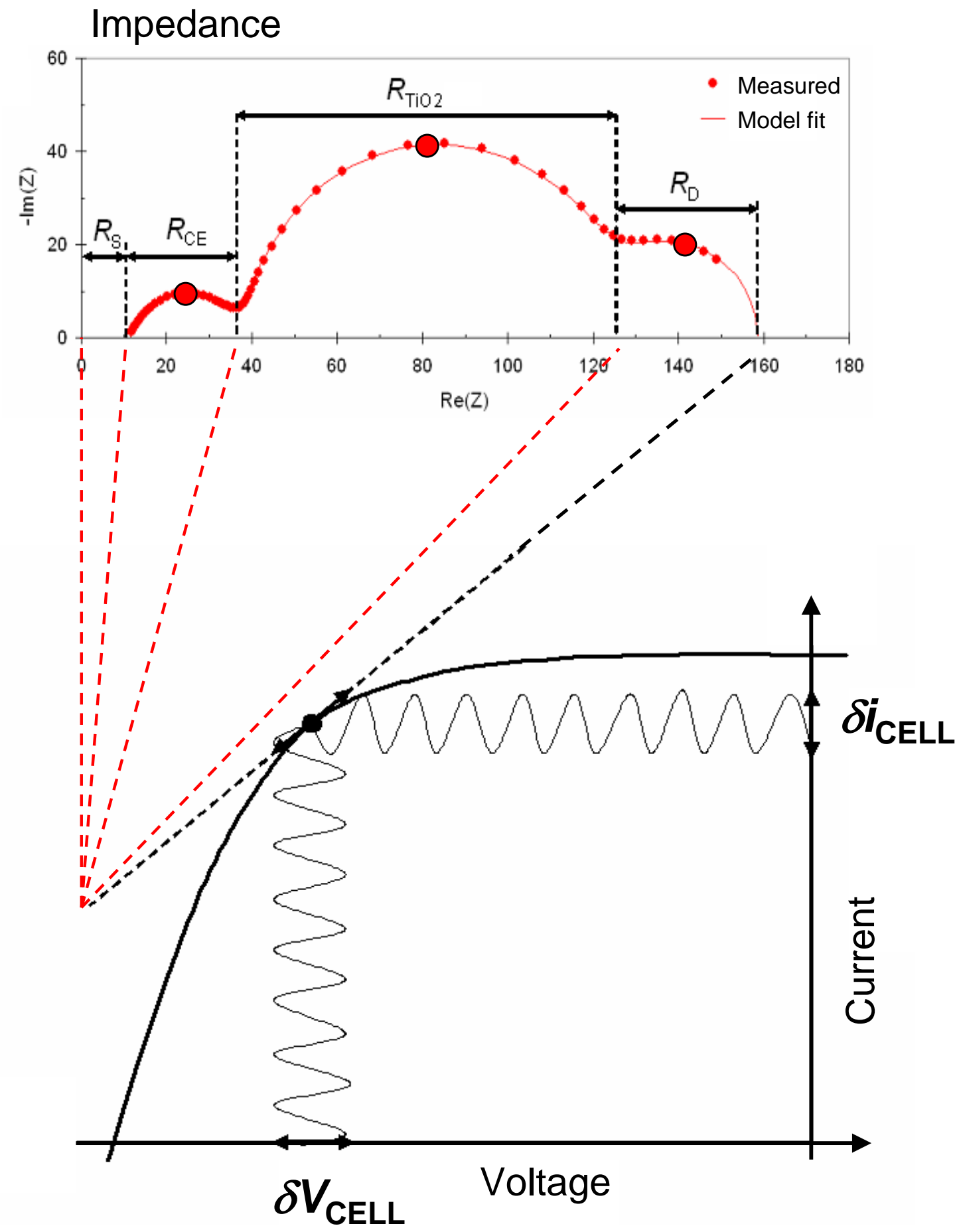

Figure 5. Schematic representation of the relation between the IV curve of DSC and its differential internal resistances measured by EIS. Individual differential resistance components of the IV curve are seen as separate impedance arcs due to their different characteristics frequencies. These characteristic frequencies correspond to the apex of each impedance arc and depend on the operating point along the IV curve. Typically $R_{\mathrm{CE}}$ is seen in the $\mathrm{kHz}$ region, $R_{\mathrm{TiO} 2}$ at $1 \mathrm{~Hz}-10 \mathrm{~Hz}$, and $R_{\mathrm{D}}$ at $0.01-1 \mathrm{~Hz}$. 
a)

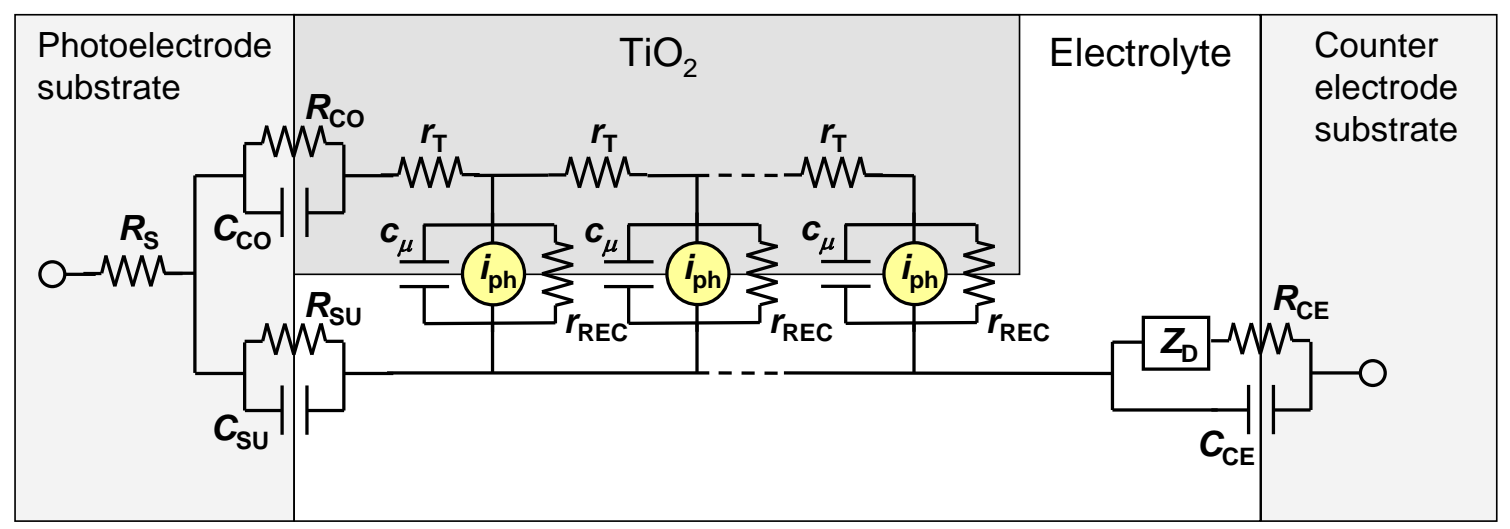

b)

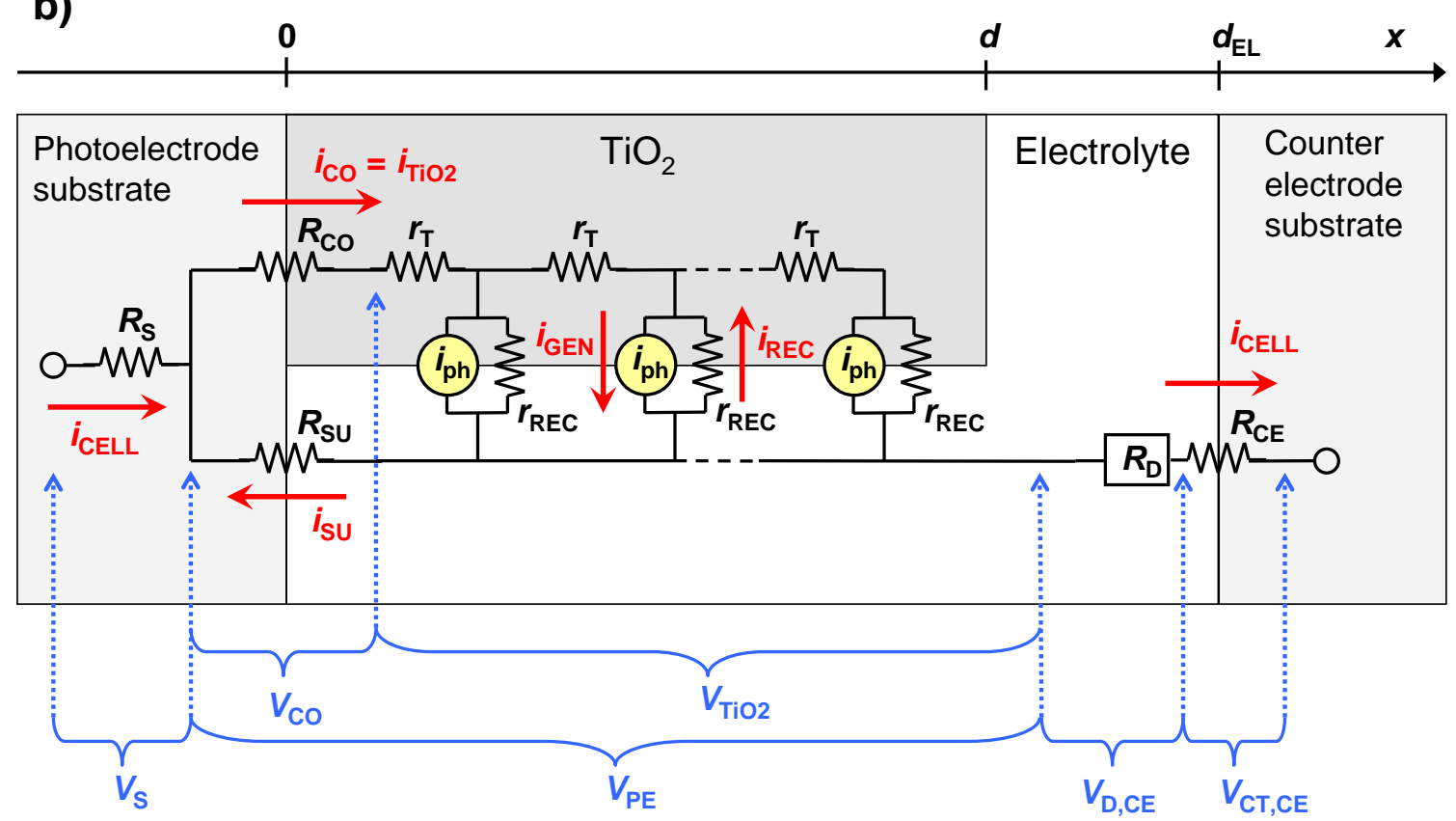

Figure 6. a) Equivalent circuit impedance model of the dye solar cell. b) Resistance model of DSC at the steady state, indicating the definition of the internal voltages and currents used in the modeling. The red arrows show the positive direction of the currents, whereas the voltages are defined as the electric potential $(\varphi)$ at the left hand side $v s$ at right hand side of the component, $V_{\mathrm{i}}=\varphi_{\mathrm{LEFT}}-\varphi_{\mathrm{LEFT}}$. The cell voltage is thus $V_{\mathrm{CELL}}=\varphi_{\mathrm{PE}}-\varphi_{\mathrm{CE}}$ and negative at the open circuit condition, whereas the short circuit current density is positive: $i_{\mathrm{SC}}=i_{\mathrm{CELL}}\left(V_{\mathrm{CELL}}=\right.$ $0)=i_{\mathrm{GEN}}-i_{\mathrm{REC}}-i_{\mathrm{SU}}$

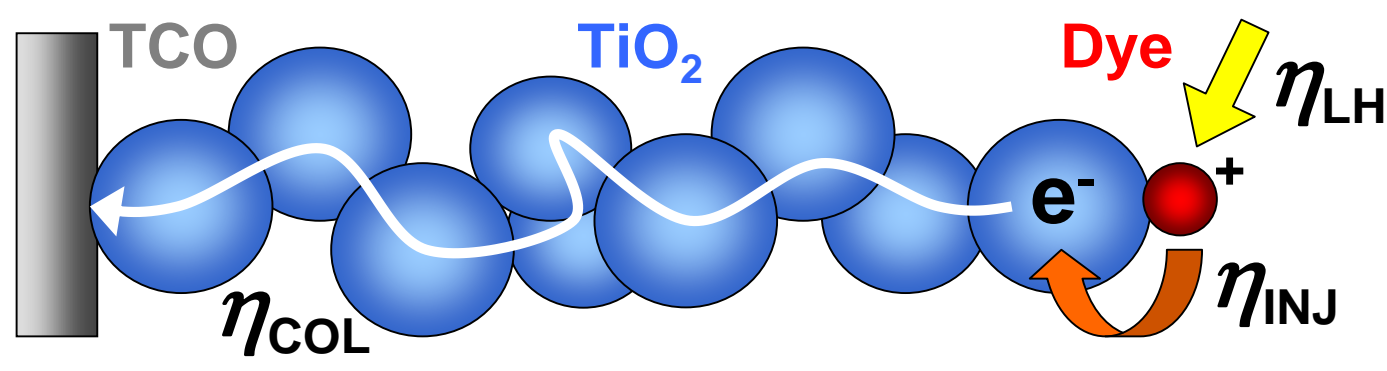


Figure 7. The photocurrent in the dye solar cell is determined by the quantum efficiencies of light harvesting $\left(\eta_{\mathrm{LH}}\right)$, electron injection $\left(\eta_{\mathrm{INJ}}\right)$, and electron collection $\left(\eta_{\mathrm{COL}}\right)$ processes.

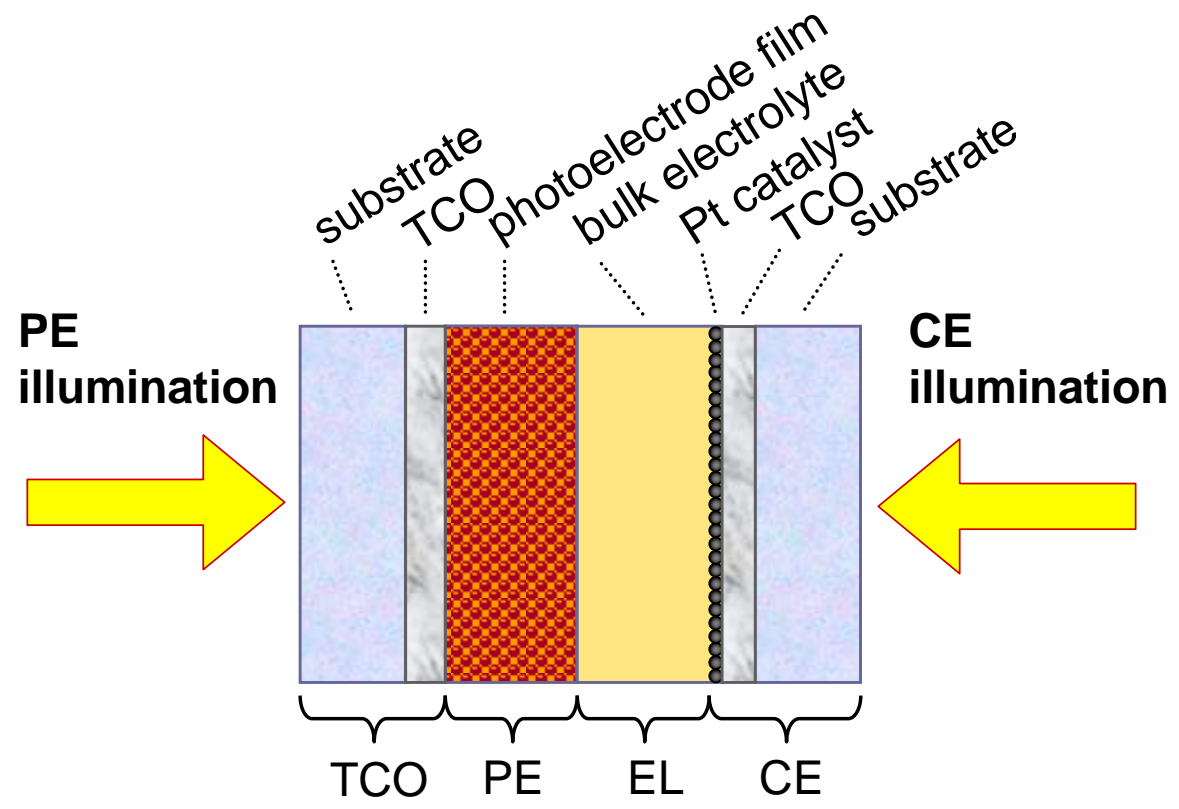

Figure 8. Optical layer structure of DSC.

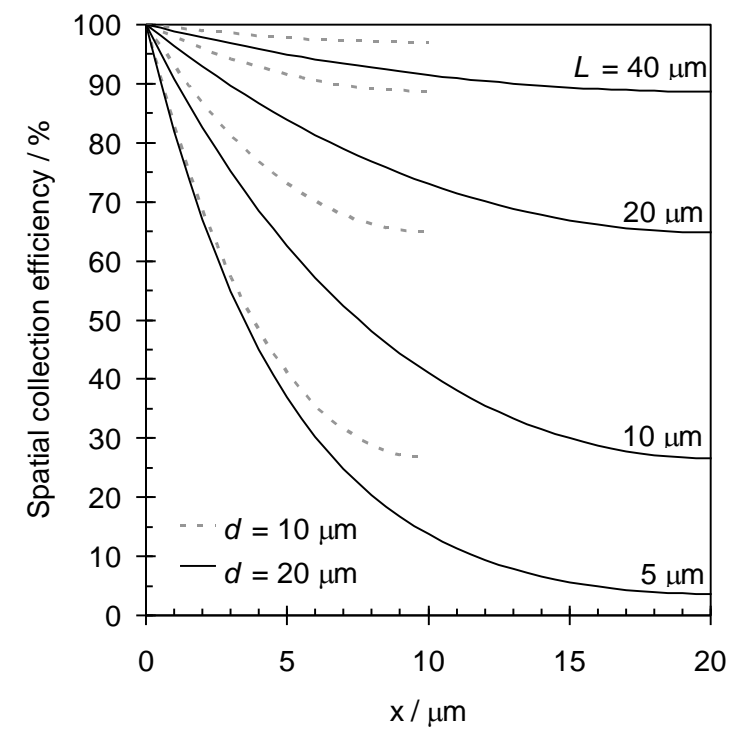

Figure 9. Spatial electron collection efficiency (Equation 30) in 10 and $20 \mu \mathrm{m}$ thick DSC photoelectrode for four values of electron diffusion lengths $(L)$. 

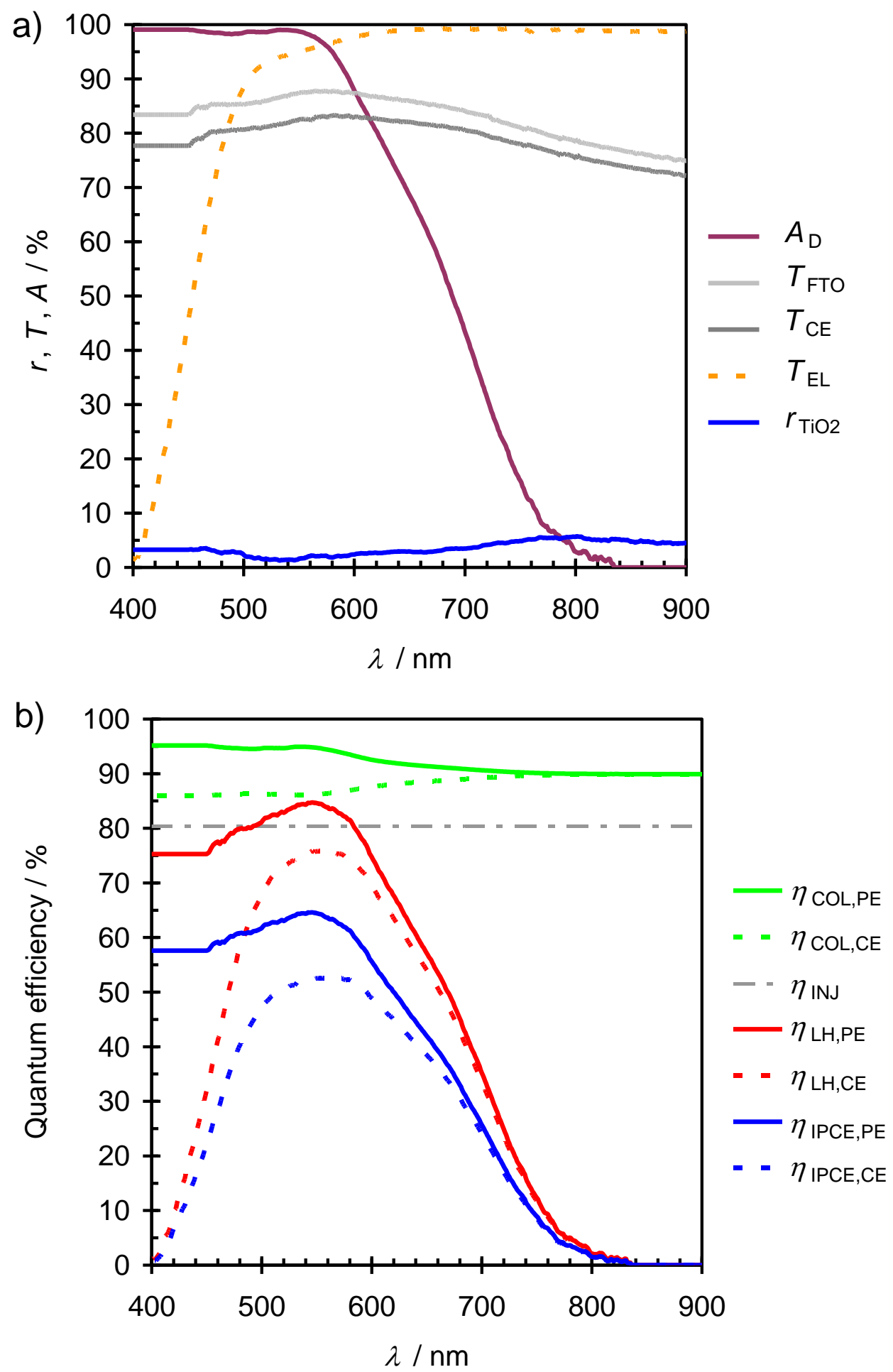

Figure 10. Typical optical performance data of DSC. The data is from ref. [38] and adjusted to the present photoelectrode and electrolyte thicknesses (Table 1). a) Transmittance and reflectance spectra used in Equations 22a-b and the absorptance spectrum of the dyed photoelectrode films, $A_{\mathrm{D}}=1-\exp \left(-\alpha_{\mathrm{D}} d\right)$; b) IPCE spectra (Equation 21) and its factors $\eta_{\mathrm{LH}}$ (Equations 21a-b), $\eta_{\mathrm{COL}}$ (Equations 28a-b), calculated based on the data in figure a) and (Table 1), and assuming $\eta_{\mathrm{INJ}}=0.804$; A fixed value is used as an estimate below $450 \mathrm{~nm}$ due to lack of accurate data in this region. 


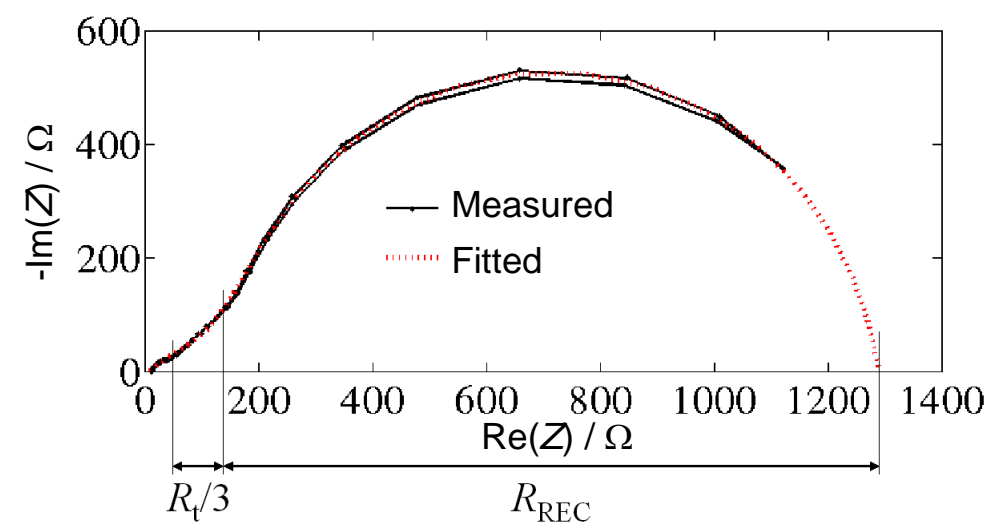

Figure 11. Measured impedance spectrum of the experimental cell (Figure 16) at $V_{\text {CELL }}=-0.5$ $\mathrm{V}$ in the dark and the fit of the equivalent circuit model of Figure $6 \mathrm{a}$ to the data, including the transmission line model (Equation $\mathrm{A} 1$ ) but neglecting $R_{\mathrm{SU}} / C_{\mathrm{SU}}, R_{\mathrm{CO}} / C_{\mathrm{CO}}$ and $Z_{\mathrm{D}}$. The photoelectrode impedance displays both the electron recombination $\left(R_{\mathrm{REC}}\right.$, apex of the arc at ca. $0.2 \mathrm{~Hz})$ and transport $\left(R_{\mathrm{T}}\right.$, characteristic shoulder in the arc at ca. $\left.6 \mathrm{~Hz}\right)$ features allowing estimation of the electron diffusion length $L=21.8 \mu \mathrm{m}$ by Equation 48 .

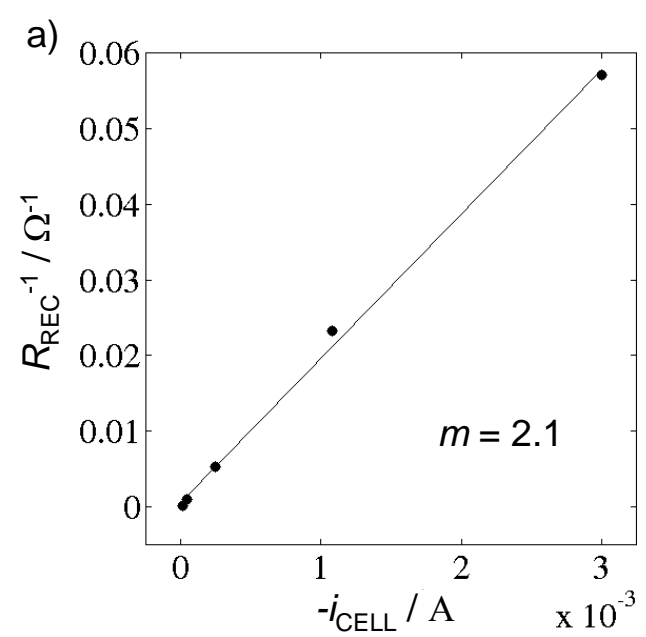

b)
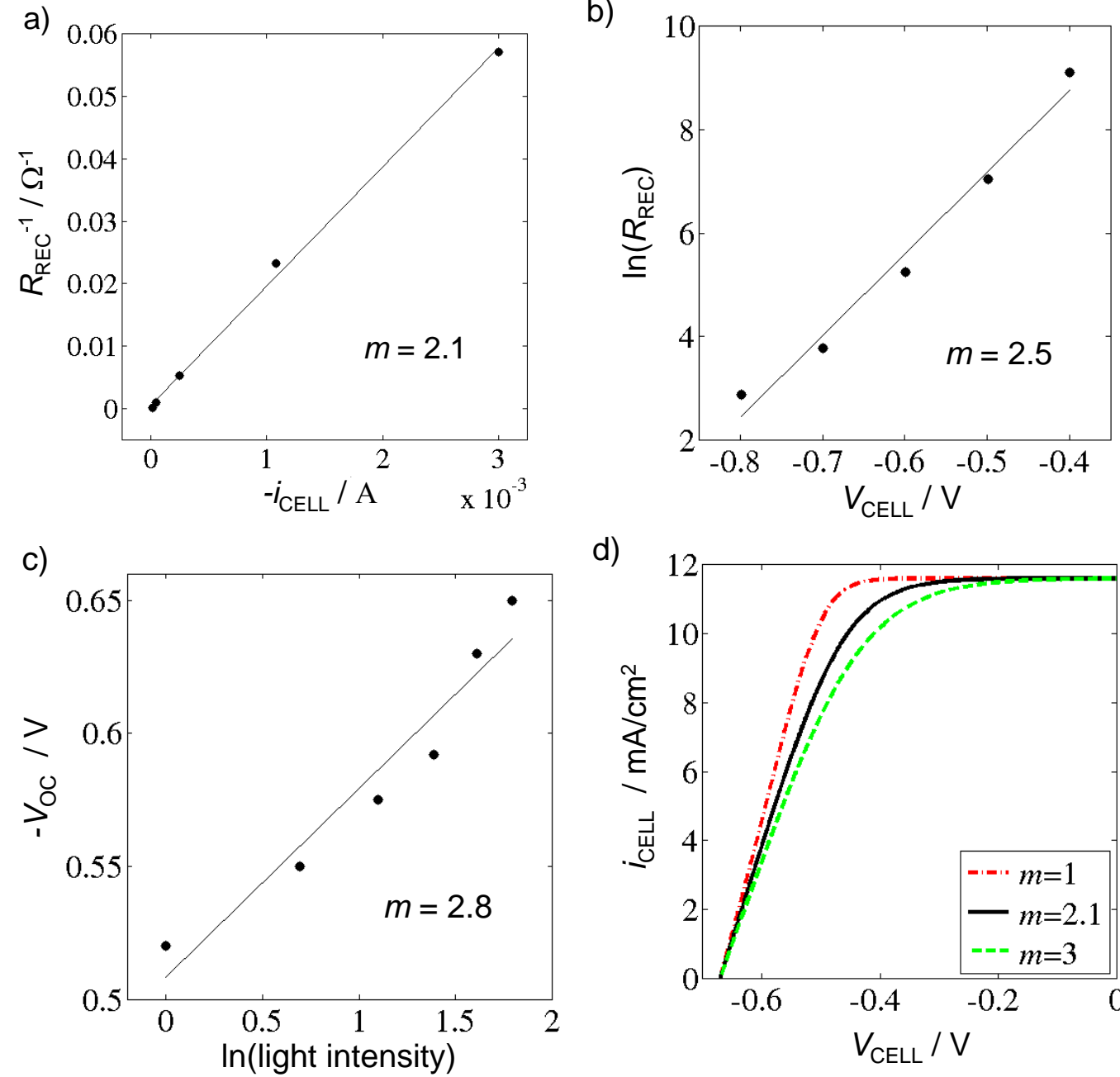

d)

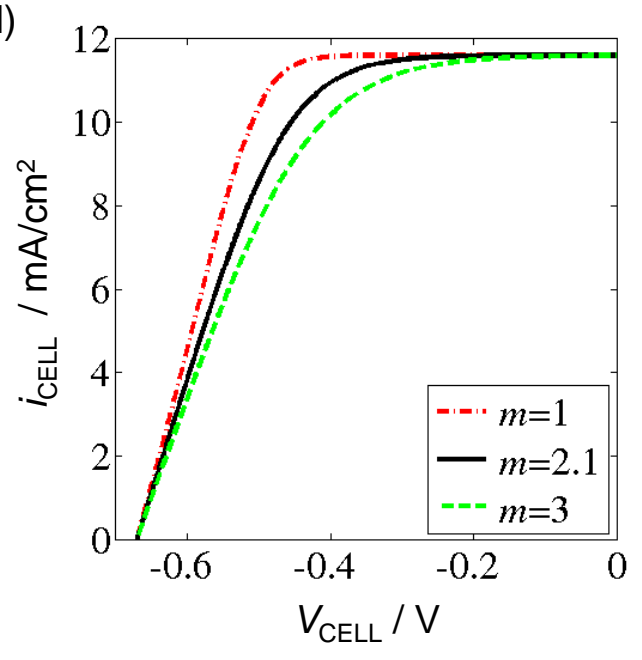

Figure 12. Experimental fingerprints of the nonideality of DSC demonstrating different methods for determining $m$ and its effect on the solar cell IV curve. a) Current dependence of $R_{\mathrm{REC}}$ in the dark (Equation 39, with $i_{\mathrm{CELL}}=-i_{\mathrm{REC}}$ ). b) Cell voltage dependence of $R_{\mathrm{REC}}$ by Equation 38 subject to the assumption that $V_{\mathrm{CELL}}=V_{\mathrm{TiO} 2}$. c) Light intensity dependence of 
$V_{\mathrm{OC}}$ by Equation 43, affected possibly by electron recombination via substrate and light induced effects. d) The effect $m$ on the solar cell IV curve for fixed $V_{\text {OC. }}$

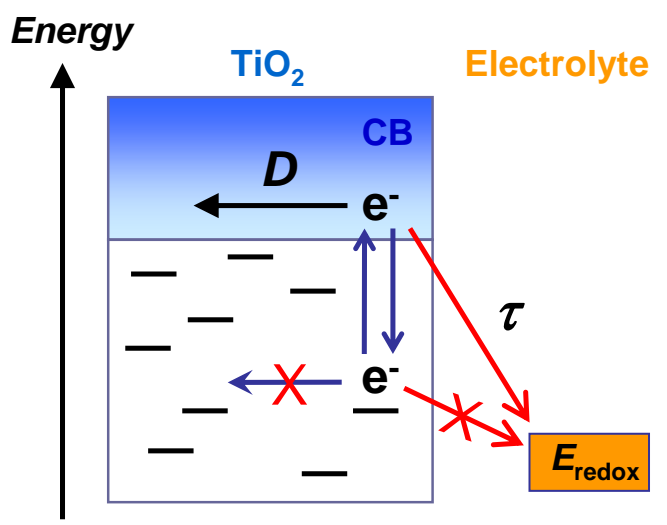

Figure 13. Electron trapping, transport and recombination processes in DSC. If trap-to-trap electron transport and direct recombination from the trap states can be neglected, electron trapping does not affect the steady state cell performance, but only the dynamic electron transport and recombination characteristics.
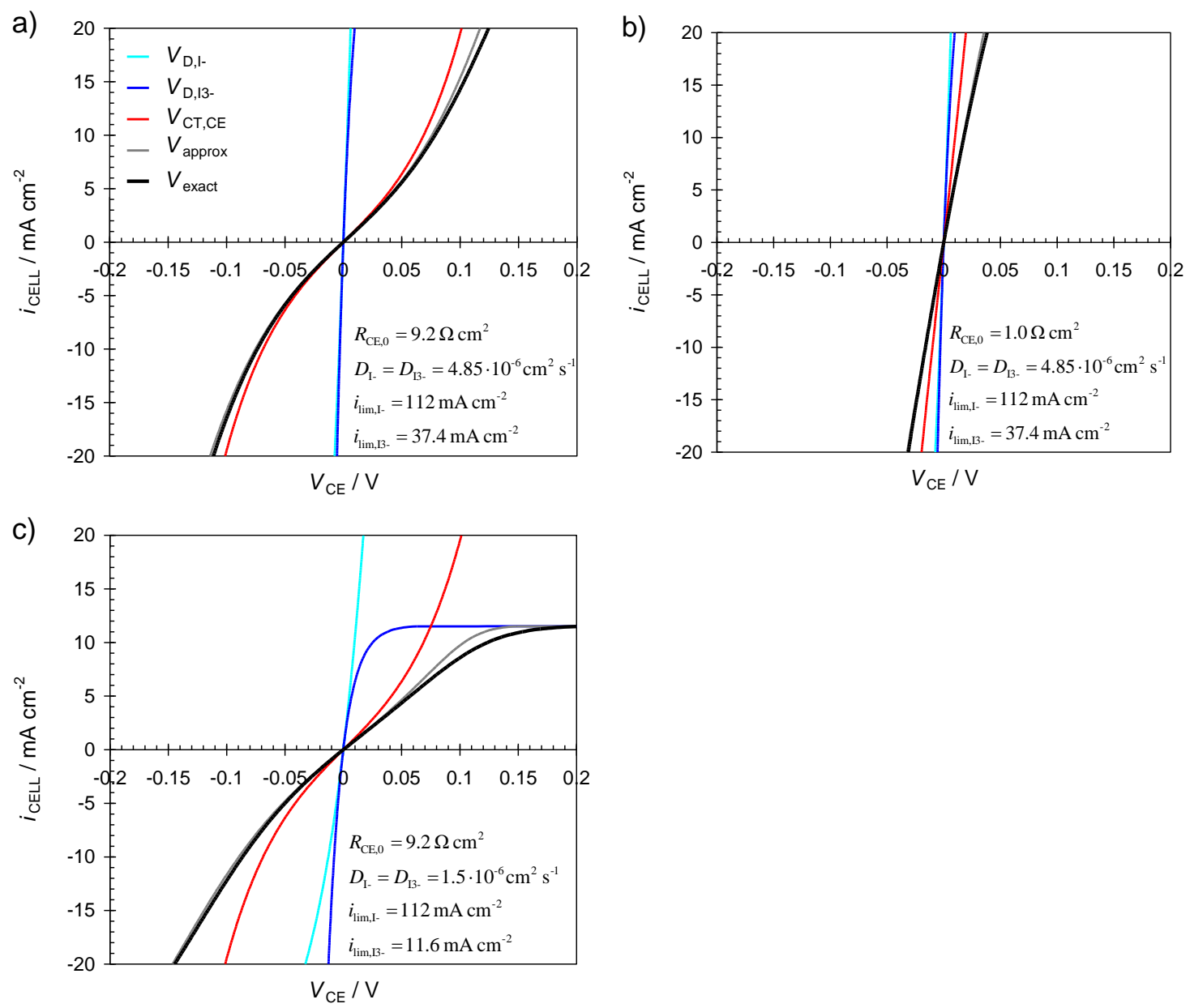

Figure 14. Calculated exact ( $\left.V_{\text {exact }}\right)$ counter electrode IV curves (Equation 59) and the partial contributions from activation overpotential $V_{\mathrm{CT}, \mathrm{CE}}$ (Equation 60), and mass transport overpotentials of tri-iodide $V_{\mathrm{D}, \mathrm{I3}-}$ (Equation 70a) and iodide $V_{\mathrm{D}, \mathrm{I}}$ (Equation $70 \mathrm{~b}$ ), and their sum $V_{\text {approx }}$ that is an approximation of Equation 59. (a) Parameters corresponding to the present experimental case (cf. Section 7); (b) same as in (a) but with excellent catalytic activity; (c) same as in (a) but with reduced electrolyte diffusion coefficient. In all cases: $\beta=$ 
$0.5, d_{\mathrm{EL}}=25 \mu \mathrm{m}, C^{*}{ }_{\mathrm{I} 3}=0.05 \mathrm{~mol} \mathrm{dm}^{-3}$, and $C_{\mathrm{I}-}^{*}=0.45 \mathrm{~mol} \mathrm{dm}^{-3}$. The traces are labeled in the legend of figure (a) in the order of increasing voltage at $5 \mathrm{~mA} \mathrm{~cm}^{-2}$.

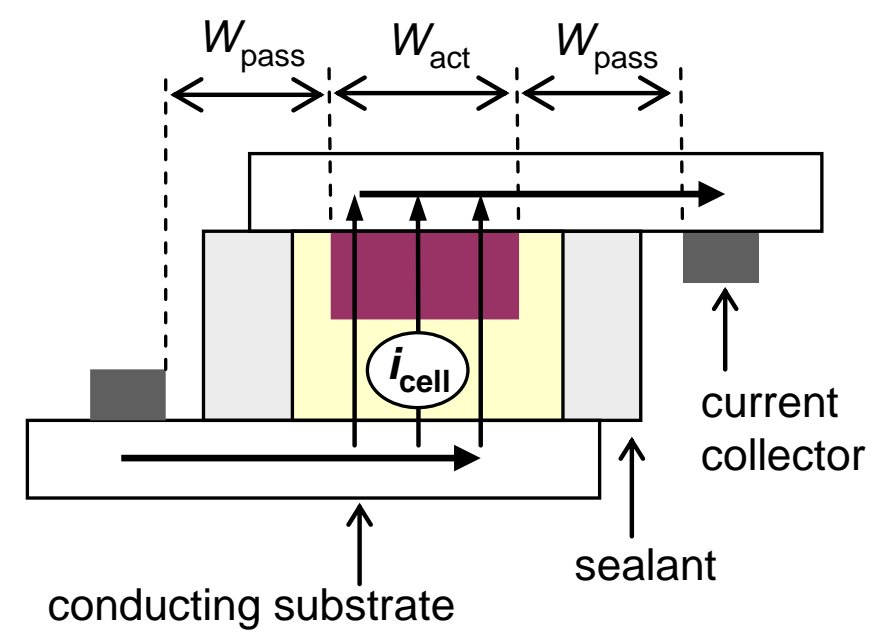

Figure 15. Schematic cross-section geometry of a symmetric small DSC. The active area extends over a length $L_{\text {act }}$ perpendicular to the paper.

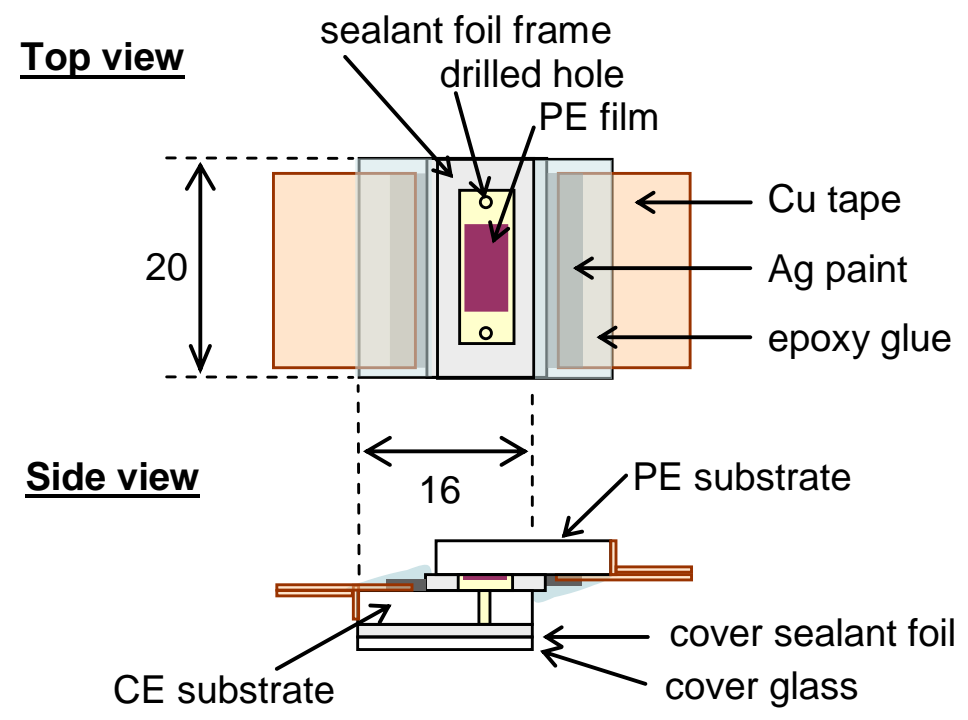

Figure 16. Construction of a typical small area laboratory test DSC. The cells were prepared on FTO-glass substrates, using nanostructured $\mathrm{TiO}_{2}$ photoelectrode, $\mathrm{N} 719$ dye, thermally platinized counter electrode, and conventional liquid electrolyte $\left(0.5 \mathrm{~mol} \mathrm{dm}^{-3} \mathrm{LiI}, 0.03 \mathrm{~mol}\right.$ $\mathrm{dm}^{-3} \mathrm{I}_{2}$ and $0.05 \mathrm{~mol} \mathrm{dm}^{-3}$ tert-butylpyridine in 3-methoxypropionitrile). The active area of the solar cell was $0.4 \mathrm{~cm}^{2}$. The cell assembly is described in more detail elsewhere.[29] 


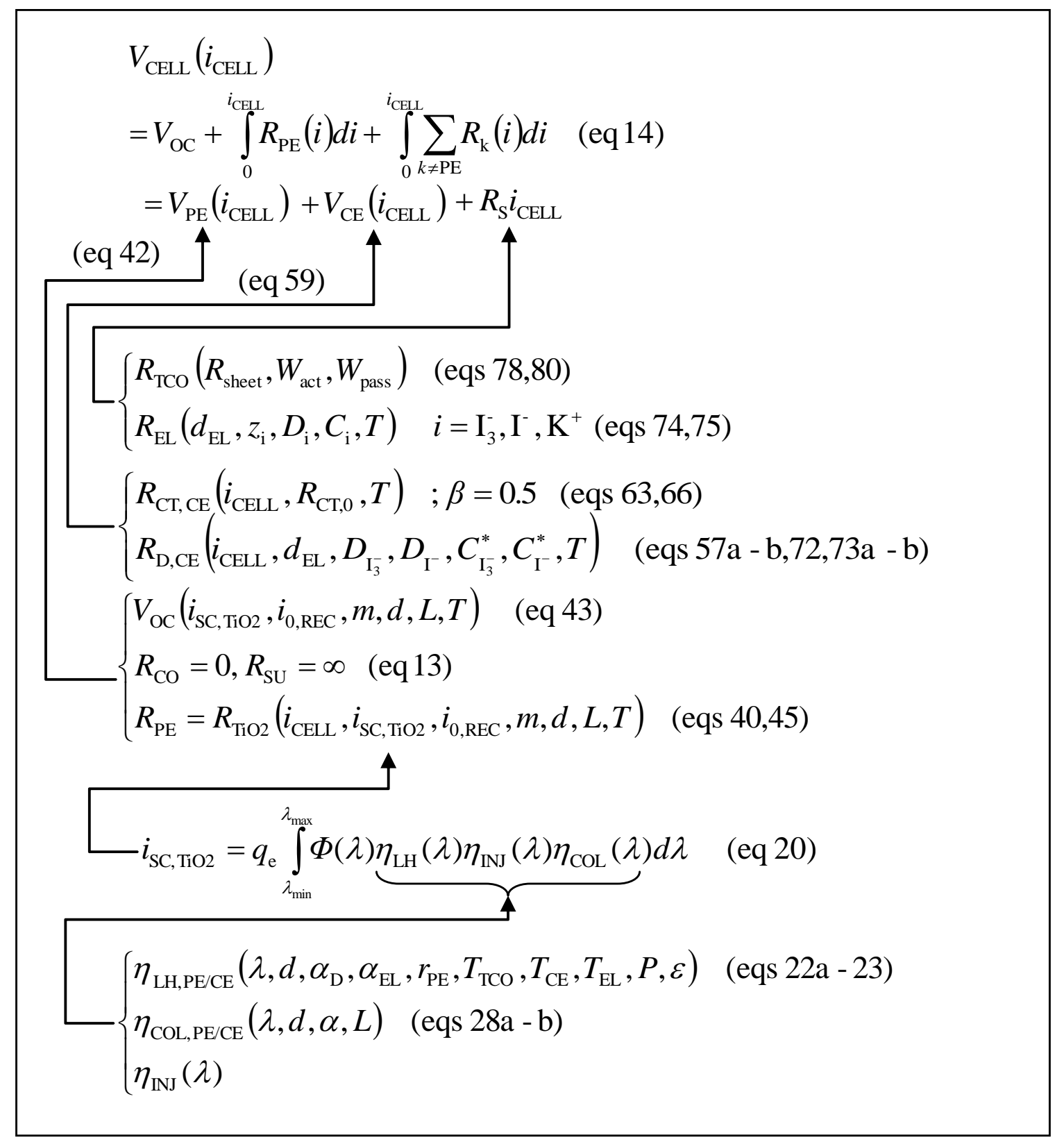

Figure 17. Structure of the physical equivalent circuit model summarizing the key equations and the relations between the model parameters. The modeling was done in three distinct domains: (1) IV modeling (third row). The solar cell IV curve is a sum of the partial IV curves of the photoelectrode and counter electrode, given by the "master" Equations 42 and 59, and the Ohmic losses due to the series resistance $R_{\mathrm{S}}$; (2) Resistance modeling (middle part). Current dependent expressions for the differential resistances, measurable by EIS, were derived from the master Equations 42 and 59. The second row recalls how they are related to the IV curve; (3) Photocurrent modeling (lower part). The photocurrent $i_{\mathrm{SC}, \mathrm{TiO} 2}$, used in the expressions for the photoelectrode resistance and IV curve, is determined by the incident photon flux $\Phi$ and the three quantum efficiencies, of which $\eta_{\mathrm{LH}}$ and $\eta_{\mathrm{COL}}$ were given a physical model while $\eta_{\mathrm{INJ}}$ was used as a parameter (that can be replaced with experimental data). 


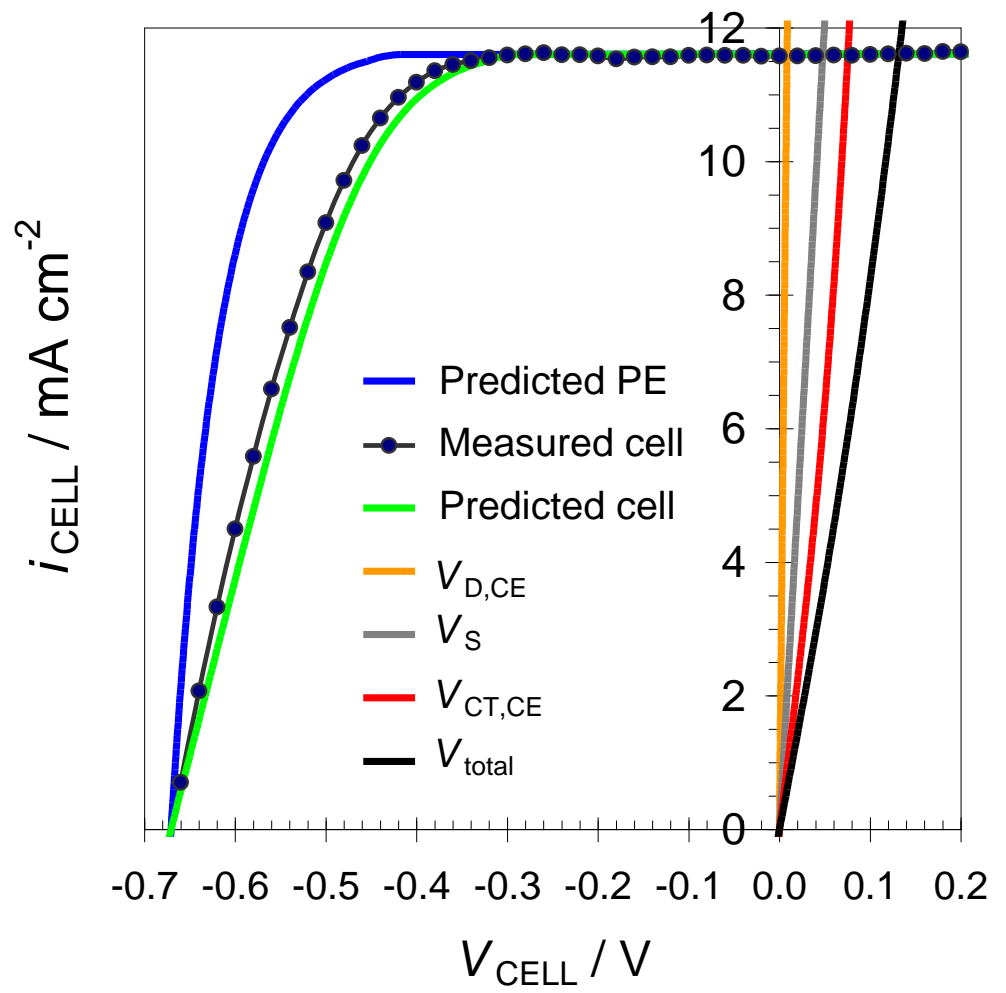

Figure 18. Comparison of IV data measured at $1000 \mathrm{~W} \mathrm{~m}^{-2} \mathrm{AM} 1.5 \mathrm{G}$ equivalent light intensity of a $4.7 \%$ efficient DSC to the predictions of a physical equivalent circuit model using parameters in Table 1 . The measured IV curve has $V_{\mathrm{oc}}=0.67 \mathrm{~V}, i_{\mathrm{SC}}=11.7 \mathrm{~mA} \mathrm{~cm}^{-2}$ and fill factor of 0.60 .

Table 1. List of model parameters and their experimentally estimated or assumed values.

\begin{tabular}{|c|c|c|c|c|}
\hline Parameter & Symbol & Value & Units & Estimation method \\
\hline \multicolumn{5}{|l|}{ Environmental conditions } \\
\hline Absolute cell temperature & $T$ & 293.15 & {$[\mathrm{~K}]$} & Assumption \\
\hline Incident photon flux & $\Phi$ & \multicolumn{3}{|c|}{$\begin{array}{l}\text { Standard AM1.5G } 1000 \mathrm{~W} \mathrm{~m}^{-2} \text { solar spectral } \\
\text { irradiance }\end{array}$} \\
\hline \multicolumn{5}{|c|}{ Cell geometry and current collection } \\
\hline $\mathrm{TiO}_{2}$ film thickness & $d$ & 12.8 & {$[\mu \mathrm{m}]$} & Profilometry \\
\hline Electrolyte thickness & $d_{\mathrm{EL}}$ & 25 & {$[\mu \mathrm{m}]$} & Micrometer \\
\hline Active area width & $W_{\text {act }}$ & 5.0 & {$[\mathrm{~mm}]$} & Ruler \\
\hline Passive area width & $W_{\text {pass }}$ & 4.0 & {$[\mathrm{~mm}]$} & Ruler \\
\hline TCO sheet resistance & $R_{\text {sheet }}$ & 7.60 & {$[\Omega]$} & $\begin{array}{l}\text { Adjusted to match } R_{\mathrm{S}} \\
\text { measured by EIS }\end{array}$ \\
\hline \multicolumn{5}{|l|}{ Optical performance } \\
\hline $\begin{array}{l}\text { Absorption coefficient of the } \\
\text { dyed } \mathrm{TiO}_{2} \text { film }\end{array}$ & $\alpha_{\mathrm{D}}$ & $\begin{array}{l}\text { Data not } \\
\text { shown }\end{array}$ & {$\left[\mathrm{m}^{-1}\right]$} & $\begin{array}{l}\text { Optical measurements } \\
\text { (Figure 10a) }\end{array}$ \\
\hline $\begin{array}{l}\text { Absorption coefficient of the } \\
\text { free electrolyte layer }\end{array}$ & $\alpha_{\mathrm{EL}}$ & $\begin{array}{l}\text { Data not } \\
\text { shown }\end{array}$ & {$\left[\mathrm{m}^{-1}\right]$} & $\begin{array}{l}\text { Optical measurements } \\
\text { (Figure 10a) }\end{array}$ \\
\hline $\begin{array}{l}\text { Porosity of the photoelectrode } \\
\text { film }\end{array}$ & $P$ & 0.5 & - & Assumption \\
\hline Optical mean path length & $\varepsilon$ & 1.5 & - & Constant. Assumption. \\
\hline $\begin{array}{l}\text { Transmittance of the } \\
\text { photoelectrode substrate }\end{array}$ & $T_{\mathrm{TCO}}$ & Figure 10 & {$[\%]$} & $\begin{array}{l}\text { Optical measurements } \\
\text { (Figure 10a) }\end{array}$ \\
\hline
\end{tabular}




\begin{tabular}{|c|c|c|c|c|}
\hline $\begin{array}{l}\text { Transmittance of the counter } \\
\text { electrode }\end{array}$ & $T_{\mathrm{CE}}$ & Figure 10 & {$[\%]$} & $\begin{array}{l}\text { Optical measurements } \\
\text { (Figure 10a) }\end{array}$ \\
\hline $\begin{array}{l}\text { Transmittance of the bulk } \\
\text { electrolyte layer }\end{array}$ & $T_{\mathrm{EL}}$ & Figure 10 & {$[\%]$} & $\begin{array}{l}\text { Optical measurements } \\
\text { (Figure 10a) }\end{array}$ \\
\hline $\begin{array}{l}\text { Reflectance of the } \\
\text { photoelectrode film } \\
\text { Photoelectrode }\end{array}$ & $r_{\mathrm{PE}}$ & Figure 10 & {$[\%]$} & $\begin{array}{l}\text { Optical measurements } \\
\text { (Figure 10a) }\end{array}$ \\
\hline Electron injection efficiency & $\eta_{\mathrm{INJ}}$ & 0.804 & - & $\begin{array}{l}\text { Constant. Adjusted to } \\
\text { match measured } i_{\mathrm{SC}} \\
\text { (Equations } 20 \text { and } 21 \text { ) }\end{array}$ \\
\hline Electron diffusion length & $L$ & 21.8 & {$[\mu \mathrm{m}]$} & $\begin{array}{l}\text { Constant. EIS in the dark } \\
\text { (Figure 11) }\end{array}$ \\
\hline $\begin{array}{l}\text { Exchange current density of } \\
\text { the electron recombination } \\
\text { reaction }\end{array}$ & $i_{\mathrm{REC}, 0}$ & $4.20 \cdot 10^{-4}$ & {$\left[\mathrm{~A} \mathrm{~m}^{-2}\right]$} & $\begin{array}{l}\text { Adjusted to match } \\
\text { measured } V_{\mathrm{OC}} \text { (Equation } \\
\text { 43) }\end{array}$ \\
\hline Ideality factor & $m$ & 2.1 & - & $\begin{array}{l}\text { EIS (Figure } 12 \mathrm{a} \text {, } \\
\text { Equations } 39 \text { and } 40 \text { ) }\end{array}$ \\
\hline \multicolumn{5}{|l|}{ Electrolyte } \\
\hline $\begin{array}{l}\text { Equilibrium concentration of } \\
\text { tri-iodide }\end{array}$ & $C_{\mathrm{I} 3} *$ & 0.05 & {$\left[\mathrm{~mol} \mathrm{dm}^{-3}\right]$} & By preparation \\
\hline $\begin{array}{l}\text { Equilibrium concentration of } \\
\text { iodide }\end{array}$ & $C_{\mathrm{I}-} *$ & 0.45 & {$\left[\mathrm{~mol} \mathrm{dm}^{-3}\right]$} & By preparation \\
\hline $\begin{array}{l}\text { Equilibrium concentration of } \\
\text { cation }\end{array}$ & $C_{\mathrm{K}+}{ }^{*}$ & 0.5 & {$\left[\mathrm{~mol} \mathrm{dm}^{-3}\right]$} & By preparation \\
\hline $\begin{array}{l}\text { Tri-iodide diffusion } \\
\text { coefficient }\end{array}$ & $D_{\mathrm{I} 3-}$ & $4.85 \cdot 10^{-6}$ & {$\left[\mathrm{~cm}^{2} \mathrm{~s}^{-1}\right]$} & $\begin{array}{l}\text { Limiting current } \\
\text { measurement }[85]\end{array}$ \\
\hline Iodide diffusion coefficient & $D_{\text {I- }}$ & $4.85 \cdot 10^{-6}$ & {$\left[\mathrm{~cm}^{2} \mathrm{~s}^{-1}\right]$} & Assumed equal to $D_{\mathrm{I}^{-}}$ \\
\hline $\begin{array}{l}\text { Cation diffusion coefficient } \\
\text { Counter electrode }\end{array}$ & $D_{\mathrm{K}+}$ & $4.85 \cdot 10^{-6}$ & {$\left[\mathrm{~cm}^{2} \mathrm{~s}^{-1}\right]$} & Assumed equal to $D_{13}-$ \\
\hline $\begin{array}{l}\text { Charge transfer resistance at } \\
\text { zero voltage }\end{array}$ & $R_{\mathrm{CE}, 0}$ & 9.2 & {$\left[\Omega \mathrm{cm}^{2}\right]$} & EIS in symmetric cell ${ }^{[85]}$ \\
\hline Symmetry factor & $\beta$ & 0.5 & - & Assumption \\
\hline
\end{tabular}


((Author bio(s), max. 75 words))

Janne Halme is a researcher at the Helsinki University of Technology (Finland) where he obtained his D.Sc. (Tech.) degree in engineering physics in 2009. He has carried out both fundamental and applied research of nanostructured dye solar cells focusing on the development of better understanding and tools for device modeling, characterization, and design of low-cost flexible dye solar cells.

\section{Author Photograph(s)}

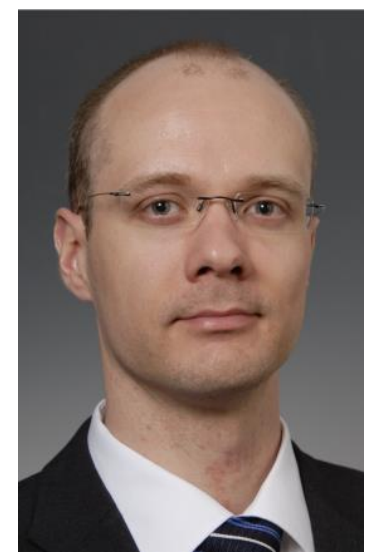

Janne Halme 
The table of contents entry should be fifty to sixty words long, written in the present tense, and refer to the chosen figure.

The key physical parameters dictating the photovoltaic performance of nanostructured dye solar cells (DSCs) can be determined using widely available experimental techniques: optical characterization, spectral quantum efficiency measurements, and impedance spectroscopy; and linked together using a complete analytical DSC device model facilitating interpretation and prediction of the current - voltage curve and energy conversion efficiency of DSCs.

\section{Keyword (see list)}

Solar Cells

C. Author-Two, D. E. F. Author-Three, A. B. Corresponding Author*((same order as byline))

Janne Halme*, Paula Vahermaa, Kati Miettunen, Peter Lund

Title ((no stars))

Device physics of dye solar cells

ToC figure ((55 mm broad, $50 \mathrm{~mm}$ high, or $110 \mathrm{~mm}$ broad, $20 \mathrm{~mm}$ high))

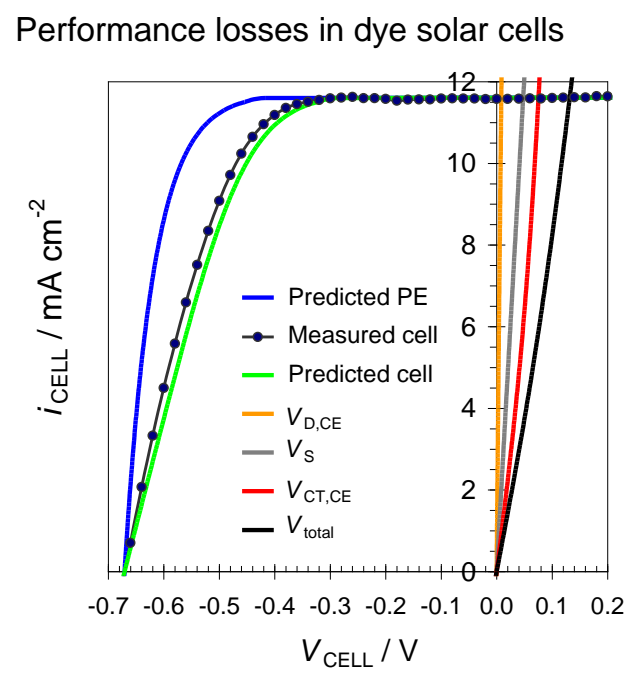

Quantum efficiencies determine the photocurrent

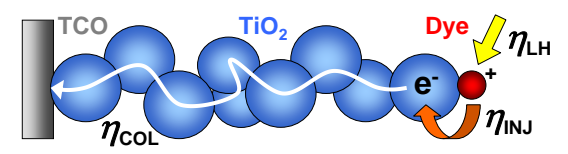

Voltage losses can be factored by impedance

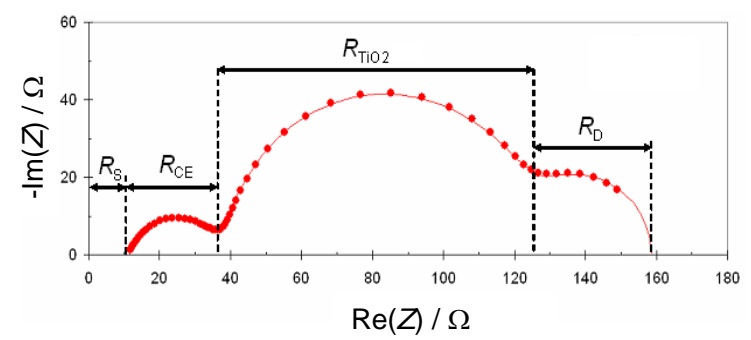

